\title{
Acid-Catalyzed Versus Thermally Induced C1-C1' Bond Cleavage in 1,1'-Bi-2-naphthol: An Experimental and Theoretical Study
}

\author{
Alexander M. Genaev, Lyudmila N. Shchegoleva, George E. Salnikov, Andrey V. Shernyukov, Leonid A. Shundrin, Inna K. Shundrina, \\ Zhongwei Zhu, and Konstantin Yu. Koltunov*
}

*E-mail: koltunov@ catalysis.ru

\section{Table of Contents}

Figure S1. Kinetics of cleavage of 1 in $\mathrm{TfOH}-\mathrm{CD}_{2} \mathrm{Cl}_{2}(2: 1 \mathrm{v} / \mathrm{v})$ at $-5^{\circ} \mathrm{C}$

Figure S2. ${ }^{1} \mathrm{H}$ NMR $(600 \mathrm{MHz})$ spectra of $\mathbf{1}$ in $\mathrm{TfOH}-\mathrm{CD}_{2} \mathrm{Cl}_{2}(2: 1 \mathrm{v} / \mathrm{v})$ at $-5{ }^{\circ} \mathrm{C}$ (time course)

Signal assignments and NMR spectra of the mixture of ions obtained in the reaction of 1 with $\mathrm{TfOH}-\mathrm{CD}_{2} \mathrm{Cl}_{2}$ for $1 \mathrm{~h}$ at $-5{ }^{\circ} \mathrm{C}$.

Figure S3. EPR spectra of $\mathbf{1}$ in triflic acid.

Figure S4. FOD plot for transition state $3 \mathbf{b}^{\neq}$

Results of CAS(6X6)/cc-pVDZ calculations of $\mathbf{3 b}$ and $\mathbf{3} \mathbf{b}^{\#}$

Results of DFT/PBE/ $\Lambda 1$ calculations of $\mathbf{3 c}, \mathbf{8}$ and $\mathbf{9}$

Thermogravimetric analysis (TGA) and differential scanning calorimetry (DSC) measurements for $\mathbf{1}$ and $\mathbf{5}$.......

* To view the original 1D and 2D $\left({ }^{1} \mathrm{H}-{ }^{13} \mathrm{C}\right.$ HSOC, ${ }^{1} \mathrm{H}-{ }^{1} \mathrm{H}$ COSY, ${ }^{1} \mathrm{H}-{ }^{1} \mathrm{H}$ ROESY correlations and $\left.\mathrm{HMBC}\right)$ NMR spectra, first of all, unzip the ZIP file in any suitable place. The archive contains the raw NMR dataset in the Bruker TOPSPIN format. To keep the archive size under reasonable bounds, the processed data were deleted, but the complete processing parameter sets and the annotated peak lists are left on place. The only action needed to recreate the processing data in TOPSPIN is to repeat fourier transformation in each expno/procno of the dataset, with the command efp for 1D data, $\mathrm{xfb}$ for 2D data. Then, to view spectrum annotations, enable peak labels and peak annotations in the TOPSPIN Spectra Display Properties.

To view the annotated NMR spectra and assigned peak tables in CCPNMR Suite, unzip the ZIP archive, recreate the processed data in Bruker TOPSPIN (see above), and open the project binol 3 used by us for structure elucidation in the CCPNMR (release 2.4.x) analysis program. CCPNMR Suite is an open source project and can be freely downloaded from https://www.ccpn.ac.uk/v2software/software/analysis/. 
Figure S1. Kinetics of cleavage of 1 in TfOH- $-\mathrm{CD}_{2} \mathrm{Cl}_{2}(2: 1 \mathrm{v} / \mathrm{v})$ at $-5{ }^{\circ} \mathrm{C}$

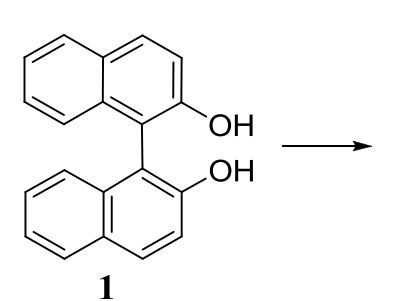

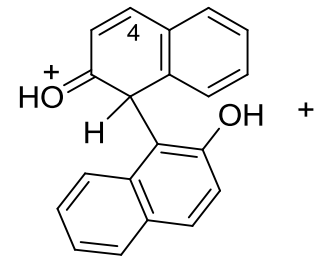

2

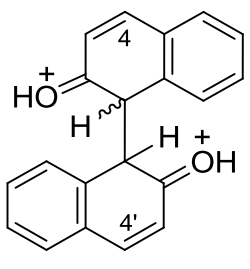

3a-c

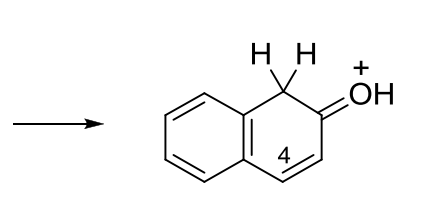

$\mathbf{N}$

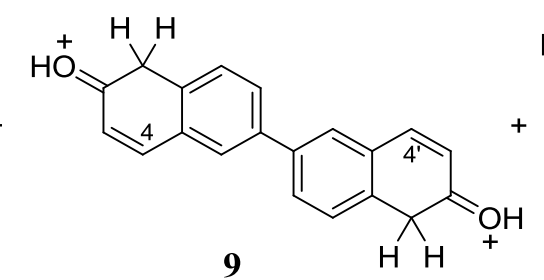

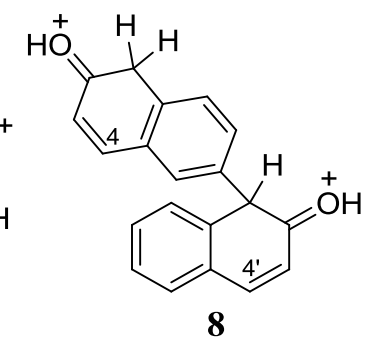

8

Reaction of $\mathbf{1}$ with TfOH gives initially a complex mixture of ions, such as $\mathbf{2}$, 3a-c, and their whole set is denoted as $\mathbf{B}$. The ${ }^{1} \mathrm{H}$ NMR signals of these species are strongly broadened due to chemical exchange between them and are overlapped with the signals of the reaction products $\mathbf{N}, \mathbf{8}$, and $\mathbf{9}$. This makes it difficult to determine the relative concentration of $\mathbf{B}$ by a regular integration of NMR signals. Hence, the spectral region of $\mathrm{H}^{4}$ protons was chosen as the most convenient for integration. The contribution of the monoprotonated form $\mathbf{2}$, having only one proton in the integration region, is rather low (not more than 0.1 ) and therefore it is neglected. This distorts the estimated value of $\Delta \mathrm{G}^{\#}$ by not more than $0.1 \mathrm{kcal} \mathrm{mol}^{-1}$. Fortunately, the $\mathrm{H}^{4}$ signal of $\mathbf{N}$ is distinctively marked and can be accurately integrated. Moreover, the $\mathrm{H}^{4}$ signal area of $\mathbf{N}$ was found to be proportional to the sum of that of $\mathbf{8 , 9}$ and minor unidentified impurities during the whole reaction course (in the ratio of $\sim 1: 1$ ). This allowed estimating the total amount of products derived from $\mathbf{B}$ based on integration of the $\mathrm{H}^{4}$ signal of $\mathbf{N}$.

${ }^{1} \mathrm{H}$ NMR spectrum region of $\mathbf{H}^{4}$ signals at $\mathrm{t}=844 \mathrm{~s}$

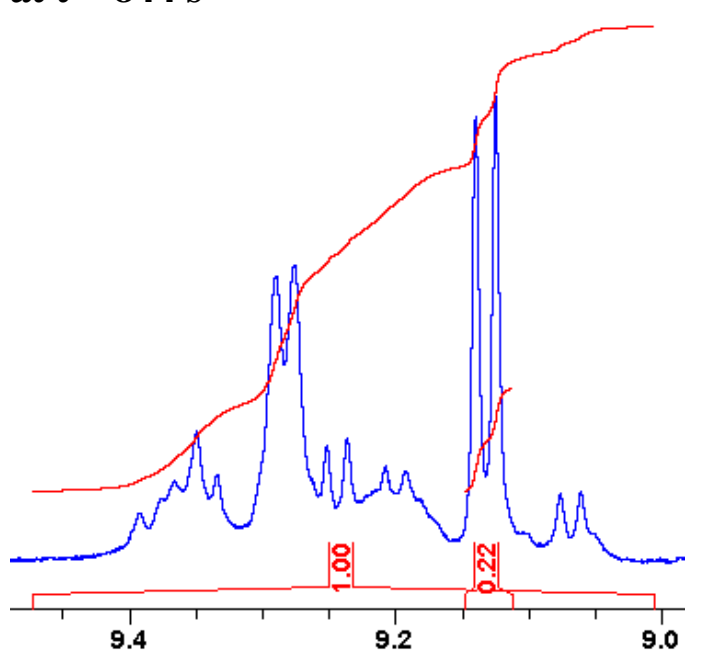

\section{Kinetic data from NMR ${ }^{a}$}

\begin{tabular}{cccc} 
time, $\mathrm{s}$ & $\mathrm{I}_{\mathbf{N}}{ }^{b}$ & $\mathrm{C}_{\mathbf{B}}{ }^{c}$ & $\ln \left(1 / \mathrm{C}_{\mathbf{B}}\right)$ \\
\hline 696 & 0.155 & 0.689 & 0.372 \\
844 & 0.219 & 0.562 & 0.576 \\
1076 & 0.286 & 0.427 & 0.850 \\
1352 & 0.342 & 0.317 & 1.150 \\
1740 & 0.390 & 0.219 & 1.518
\end{tabular}

${ }^{a}$ The total integral of the $\mathrm{H}^{4}$ region is taken as 1 .

${ }^{b} \mathbf{I}_{\mathbf{N}}$ is the integral intensity of the $\mathbf{N}$ peak.

${ }^{c} \mathrm{C}_{\mathbf{B}}=1-\mathrm{I}_{\mathbf{N}} / \mathrm{r}$ is the contribution of all forms of $\mathbf{B}$ in the total amount of all species; $r=0.5$ is the contribution of $\mathbf{N}$ in the total amount of all reaction products.

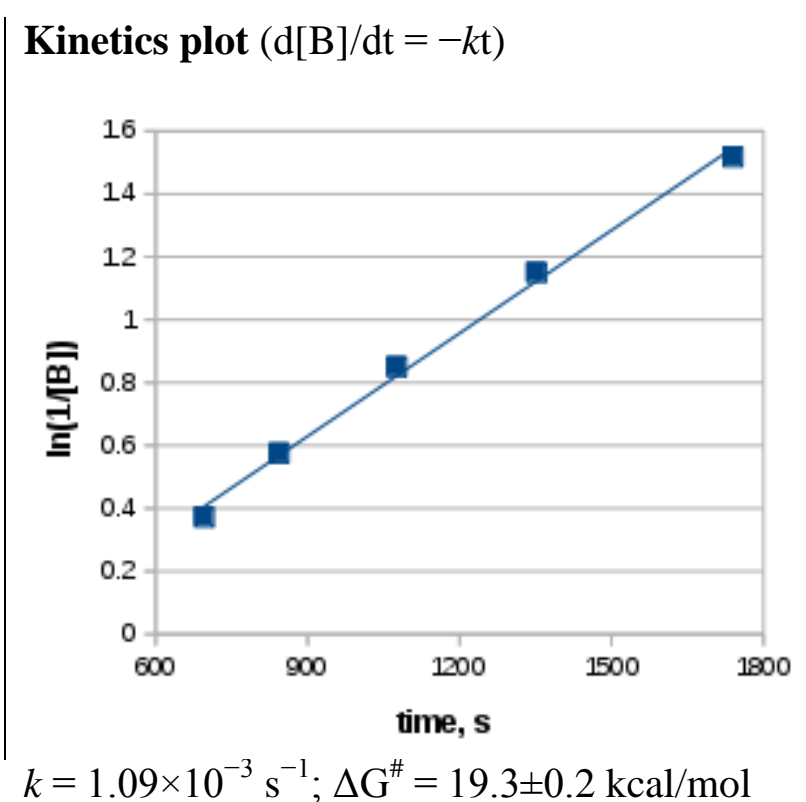


Figure S2. ${ }^{1} \mathrm{H}$ NMR $(600 \mathrm{MHz})$ spectra of $1 \mathrm{in} \mathrm{TfOH}-\mathrm{CD}_{2} \mathrm{Cl}_{2}(2: 1 \mathrm{v} / \mathrm{v})$ at $-5{ }^{\circ} \mathrm{C}$ (time course)
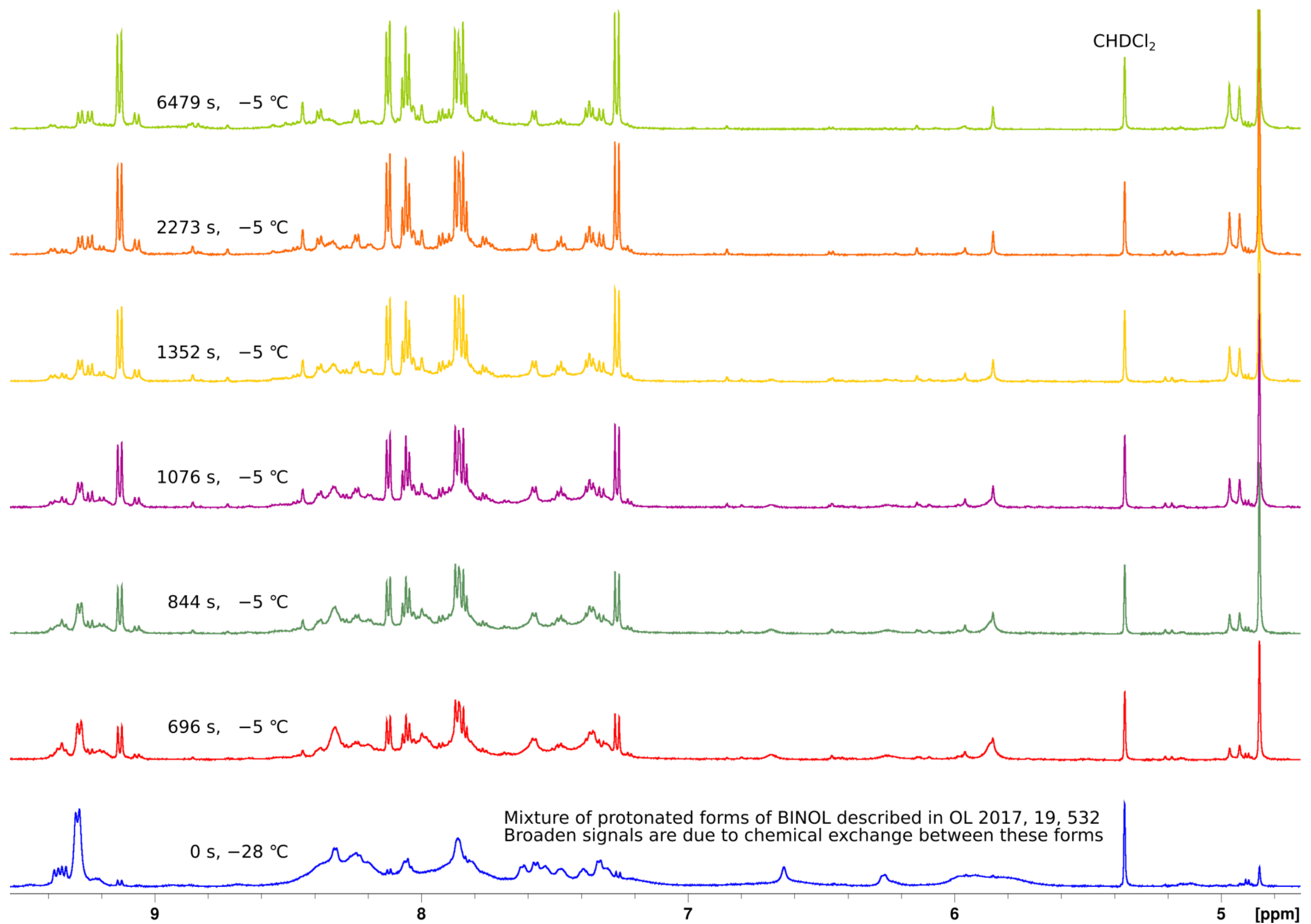
Signal assignments and NMR spectra of the mixture of ions obtained in the reaction of 1 with $\mathrm{TfOH}-\mathrm{CD}_{2} \mathrm{Cl}_{2}$ for $1 \mathrm{~h}$ at $-5{ }^{\circ} \mathrm{C}$ Spectra were taken at $-29^{\circ} \mathrm{C}$; reaction mixture: 1 (30 mg), TfOH $(930 \mathrm{mg}), \mathrm{CD}_{2} \mathrm{Cl}_{2}(140 \mathrm{mg})$; (pages S4-S16)

\section{Assignments (pages S4-S5)}

C1-protonated 2-naphthol (the corresponding signals are marked by A on spectra)

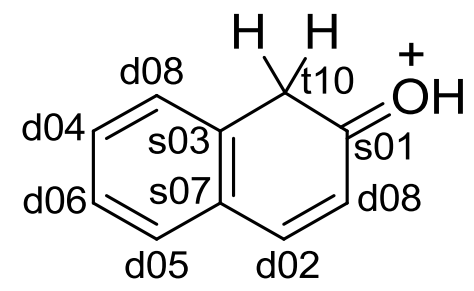

Experiment Bruker_20, 1D 13C s01 207.9

d02 174.9

s03 140.4

d04 138.5

d05 135.9

d06 130.5

s07 130.0

d08 129.7

d09 119.2

t10 40.7

Experiment Bruker_30, 1D 1H

d02-H $9.11(\mathrm{~d})$

d04-H $8.03(\mathrm{t})$

d05-H $8.10(d)$

d06-H $7.82(\mathrm{t})$

d08-H 7.85 (d)

d09-H 7.25 (d)

$\mathrm{t} 10-\mathrm{a} \quad 4.84(\mathrm{~s})$

t10-b $4.84(\mathrm{~s})$

Experiment Bruker_33, 2D 13C-1H via onebond (HSQC)

$\mathrm{t} 10-\mathrm{a}-\mathrm{t} 10$

$\mathrm{t} 10-\mathrm{b}-\mathrm{t} 10$
Experiment Bruker_35, 2D 13C-1H via onebond (HSQC arom)

d02-H - d02

d04-H - d04

d05-H - d05

d06-H - d06

d08-H - d08

d09-H - d09

Experiment Bruker_12, 2D 1H-1H via Jcoupling (COSY)

$\mathrm{d} 02-\mathrm{H}$ - t10-a(weak) t10-b(weak)

d08-H - t10-a?(weak) t10-b?(weak)

t10-a - d02-H(weak) d09-H?

t10-b - d02-H(weak) d09-H?

Experiment Bruker_14, 2D 1H-1H via Jcoupling (COSY arom)

d02-H - d09-H

d04-H - d05-H d06-H d08-H

d05-H - d04-H d06-H

d06-H - d04-H d05-H

$\mathrm{d} 08-\mathrm{H}-\mathrm{d} 04-\mathrm{H}$

d09-H - d02-H

Experiment Bruker_18, 2D 1H-1H via through-space (NOESY)

d02-H - d05-H

d05-H - d02-H

d08-H - t10-a t10-b

t10-a - d08-H

t10-b - d08-H

Experiment Bruker 32, 2D 13C-1H via Jcoupling (HMBC)

d02-H - d05 s01 s03

$\mathrm{d} 04-\mathrm{H}-\mathrm{d} 05 \mathrm{~s} 03$

d05-H - d02 d04 s03 t10(weak)

d06-H - d08 s07

d08-H - s07 t10
d09-H - s07 t10

$\mathrm{t} 10-\mathrm{a}-\mathrm{d} 08 \mathrm{~s} 01 \mathrm{~s} 03$

t10-b - d08 s01 s03

Experiment Bruker_34, 2D 13C-1H via Jcoupling (HMBC arom)

d02-H - d05 s01 s03

d04-H - d05 s03

d05-H - d02 d04 s03

d06-H - s07

d08-H - d06 s07 t10

d09-H - s07 t10

Dication 8 (the corresponding signals are marked by $\mathbf{B}$ on spectra)

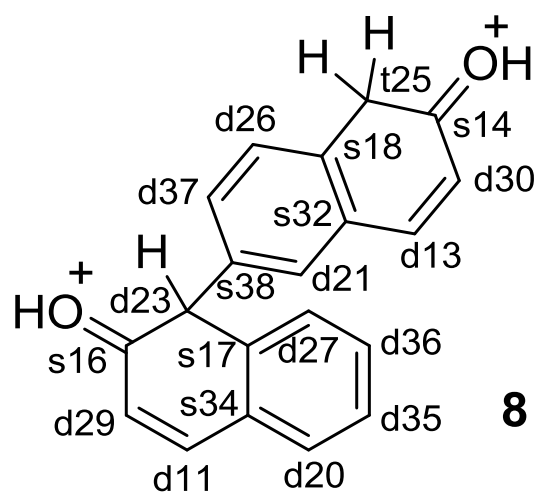

Experiment Bruker_20, 1D 13C

d11 176.2

d13 173.0

d20 137.1

d21 136.5

d23 55.2

d26 131.3

d27 130.9 $\begin{array}{ll}\text { d29 } & 118.3 \\ \text { d30 } & 120.6 \\ \text { d35 } & 131.3 \\ \text { d36 } & 139.8 \\ \text { d37 } & 138.4 \\ \text { s14 } & 209.7 \\ \text { s16 } & 207.7 \\ \text { s17 } & 144.3 \\ \text { s18 } & 140.9 \\ \text { s32 } & 131.0 \\ \text { s34 } & 129.6 \\ \text { s38 } & 136.7 \\ \text { t25 } & 40.8\end{array}$

Experiment Bruker_30, 1D 1H

d11-H $9.26(\mathrm{~d})$

d13-H 9.05 (d)

d20-H $8.22(\mathrm{~d})$

d21-H 7.98 (s)

d23-H 5.84 (s)

d26-H $7.91(\mathrm{~d})$

d27-H $7.56(d)$

d29-H 7.31 (d)

d30-H $7.34(\mathrm{~d})$

$\mathrm{d} 35-\mathrm{H} \quad 7.87(\mathrm{t})$

d36-H $8.00(\mathrm{t})$

d37-H 7.75 (d)

t25-a 4.91 (s)

t25-b $4.91(\mathrm{~s})$

Experiment Bruker 33, 2D 13C-1H via onebond (HSQC)

d23-H - d23

$\mathrm{d} 27-\mathrm{H}-\mathrm{d} 27$

d30-H - d30

$\mathrm{t} 25-\mathrm{a}-\mathrm{t} 25$

$\mathrm{t} 25-\mathrm{b}-\mathrm{t} 25$

Experiment Bruker_35, 2D 13C-1H via onebond (HSQC arom) 
d11-H - d11

d13-H - d13

d20-H - d20

d21-H - d21

d26-H - d26

d27-H - d27

d29-H - d29

d30-H - d30

d35-H - d35

d36-H - d36

d37-H - d37

Experiment Bruker_12, 2D 1H-1H via Jcoupling (COSY)

d11-H - d29-H

d13-H - d30-H

d20-H - d35-H

d26-H - d37-H

d27-H - d36-H

d29-H - d11-H

d30-H - d13-H

d35-H - d20-H

d36-H - d27-H d35-H?

d37-H - d26-H

Experiment Bruker_14, 2D 1H-1H via

Jcoupling (COSY arom)

d11-H - d29-H

d13-H - d30-H

d20-H - d35-H

d26-H - d37-H

d27-H - d36-H

d29-H - d11-H

d30-H - d13-H

d35-H - d20-H d36-H

d36-H - d27-H d35-H

d37-H - d26-H

Experiment Bruker_18, 2D 1H-1H via through-space (NOESY)

d11-H - d20-H

d13-H - d21-H

d20-H - d11-H

d21-H - d13-H d23-H

d23-H - d21-H d27-H d37-H
d26-H - t25-a t25-b

d27-H - d23-H

d37-H - d23-H

t25-a - d26-H

t25-b - d26-H

Experiment Bruker_32, 2D 13C-1H via Jcoupling (HMBC)

d11-H - d20 s16 s17

d13-H - d21 s14 s18

d20-H - d11 d36 s17

d21-H - d13 d23 d37 s18

d23-H - d21 d27 d37 s17 s34

d26-H - s32 s38 t25

d27-H - d23 d35 s34

d29-H - d23 s34

d $30-\mathrm{H}$ - s 32 t 25

$\mathrm{d} 35-\mathrm{H}-\mathrm{d} 27 \mathrm{~s} 34$

d36-H - d20 s17

d37-H - d21 d23 s 18

t25-a - s14 s18

t25-b - s14 s18

Experiment Bruker 34, 2D 13C-1H via Jcoupling (HMBC arom)

d11-H - d20 s16 s17

d13-H - d21 s18

d20-H - d11 d36 s17

d21-H - d13 d37 s18

d26-H - s 32 s 38 t 25

d27-H - d23 d35 s34

d29-H - d23 s34

d $30-\mathrm{H}-\mathrm{s} 32$ t 25

d35-H - d27 s34

d36-H - d20 s17
Dication 9 (the corresponding signals are marked by $\mathbf{C}$ on spectra)

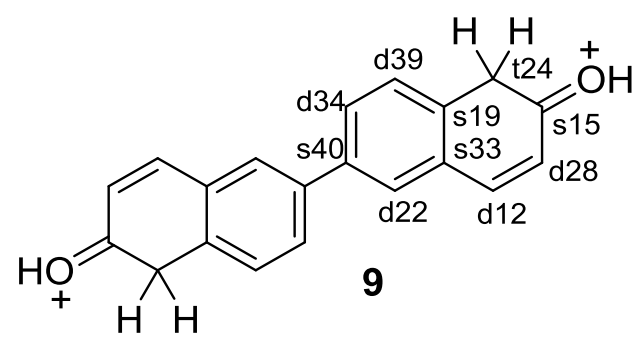

Experiment Bruker 20, 1D 13C

d12 174.2

d22 133.8

d28 120.3

d31 136.6

d39 130.6

s15 208.9

s19 140.0

s33 130.7

s40 141.0

t24 40.8

Experiment Bruker 30, 1D 1H

d12-H $9.23(d)$

d22-H $8.44(\mathrm{~s})$

d28-H $7.36(\mathrm{~d})$

d31-H 8.37 (d)

d39-H 8.02 (d)

t24-a 4.95 (s)

t24-b 4.95 (s)

Experiment Bruker 33, 2D 13C-1H via onebond (HSQC)

d28-H - d28

$\mathrm{t} 24-\mathrm{a}-\mathrm{t} 24$

$\mathrm{t} 24-\mathrm{b}-\mathrm{t} 24$

Experiment Bruker_35, 2D 13C-1H via onebond (HSQC arom)

d12-H - d12

d22-H - d22

d28-H - d28 $\mathrm{d} 31-\mathrm{H}-\mathrm{d} 31$

d39-H - d39

Experiment Bruker_12, 2D 1H-1H via Jcoupling (COSY)

d12-H - d28-H

d28-H - d12-H

d31-H - d39-H

d39-H - d31-H

Experiment Bruker_14, 2D 1H-1H via Jcoupling (COSY arom)

d12-H - d28-H

d28-H - d12-H

d31-H - d39-H

d39-H - d31-H

Experiment Bruker_18, 2D 1H-1H via through-space (NOESY)

d12-H - d22-H

d22-H - d12-H

t24-a - d39-H?

t24-b - d39-H?

Experiment Bruker_32, 2D 13C-1H via Jcoupling (HMBC)

d12-H - d22 s15 s19

d22-H - d12 d31

d28-H - s33 t24

d31-H - d22 s19

d39-H - s33 t24

t24-a - d39? s15 s19 s33?

t24-b - d39? s15 s19 s33?

Experiment Bruker 34, 2D 13C-1H via Jcoupling (HMBC arom)

d12-H - d22 s19

d22-H - d12 d31 s19 s40

$\mathrm{d} 28-\mathrm{H}$ - s $33 \mathrm{t} 24$

d31-H - d22 s19 s40

d39-H - s40 t24 
${ }^{13} \mathrm{C}\left\{{ }^{1} \mathrm{H}\right\}$ NMR spectrum $(150 \mathrm{MHz})$ of the mixture of $\mathrm{C} 1$-protonated 2-naphthol and dications $\mathbf{8}, \mathbf{9}$ in $\mathrm{TfOH}-\mathrm{CD}_{2} \mathrm{Cl}_{2}$ (pages $\mathrm{S} 6-\mathrm{S} 11$ )

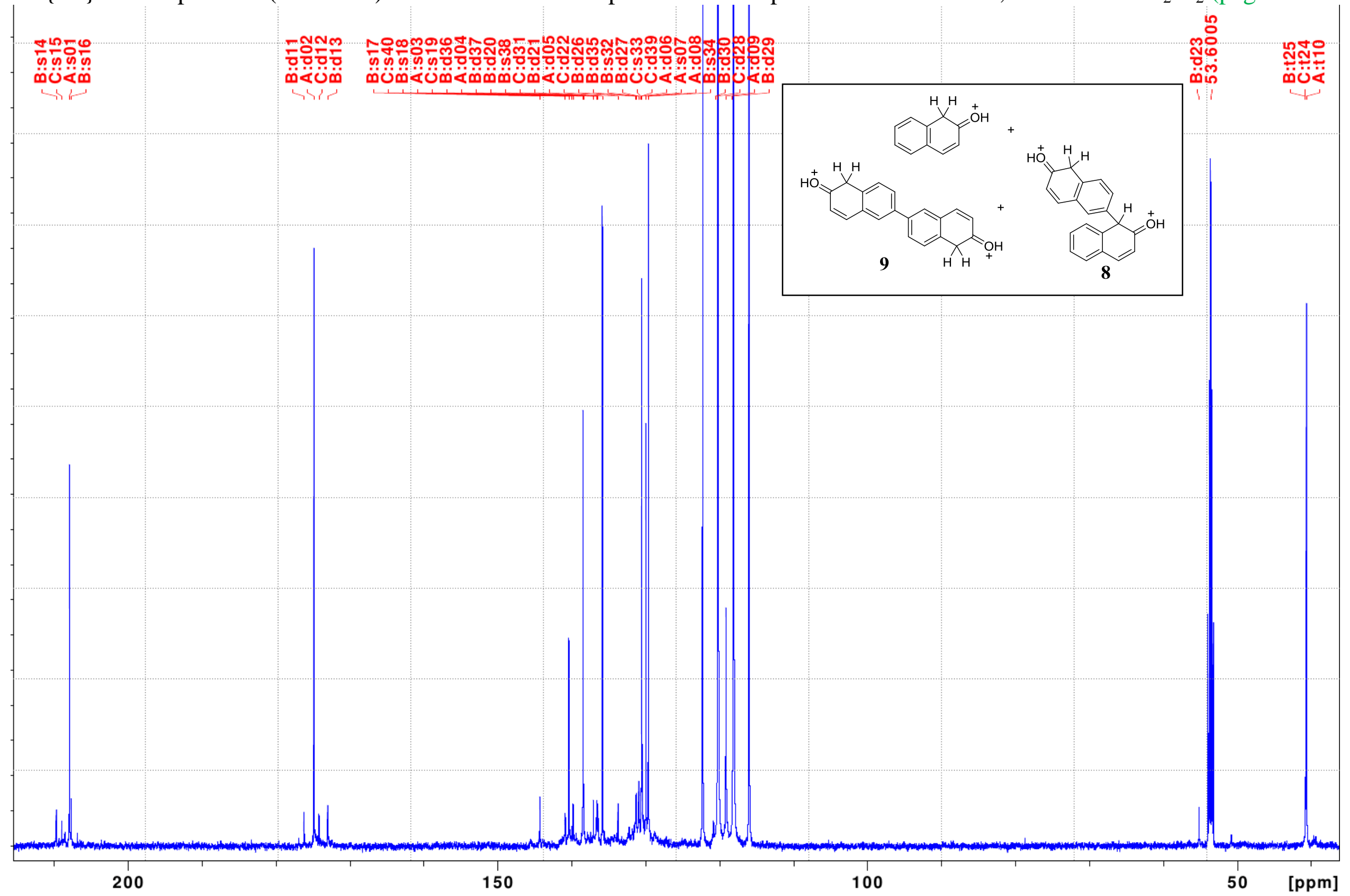




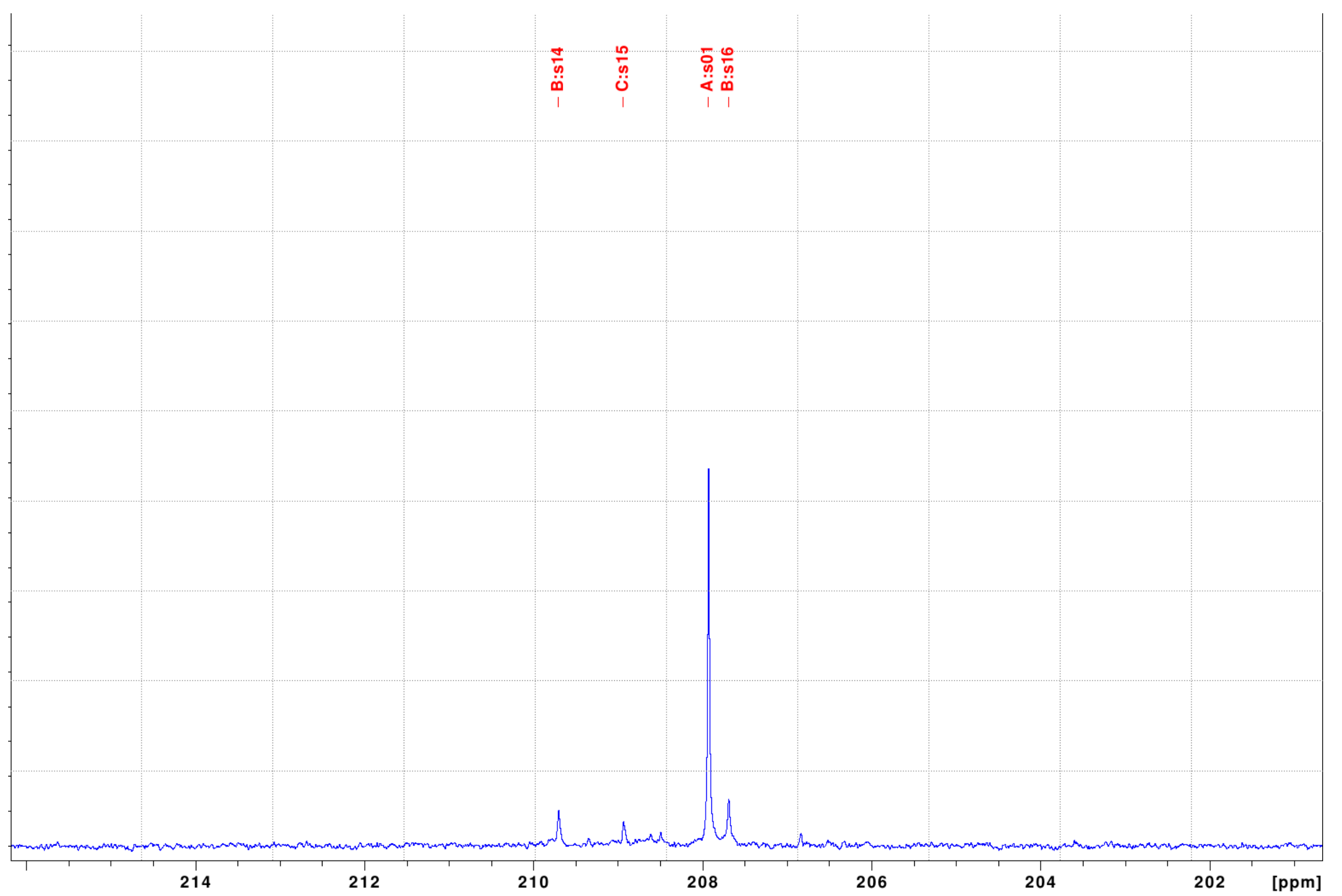




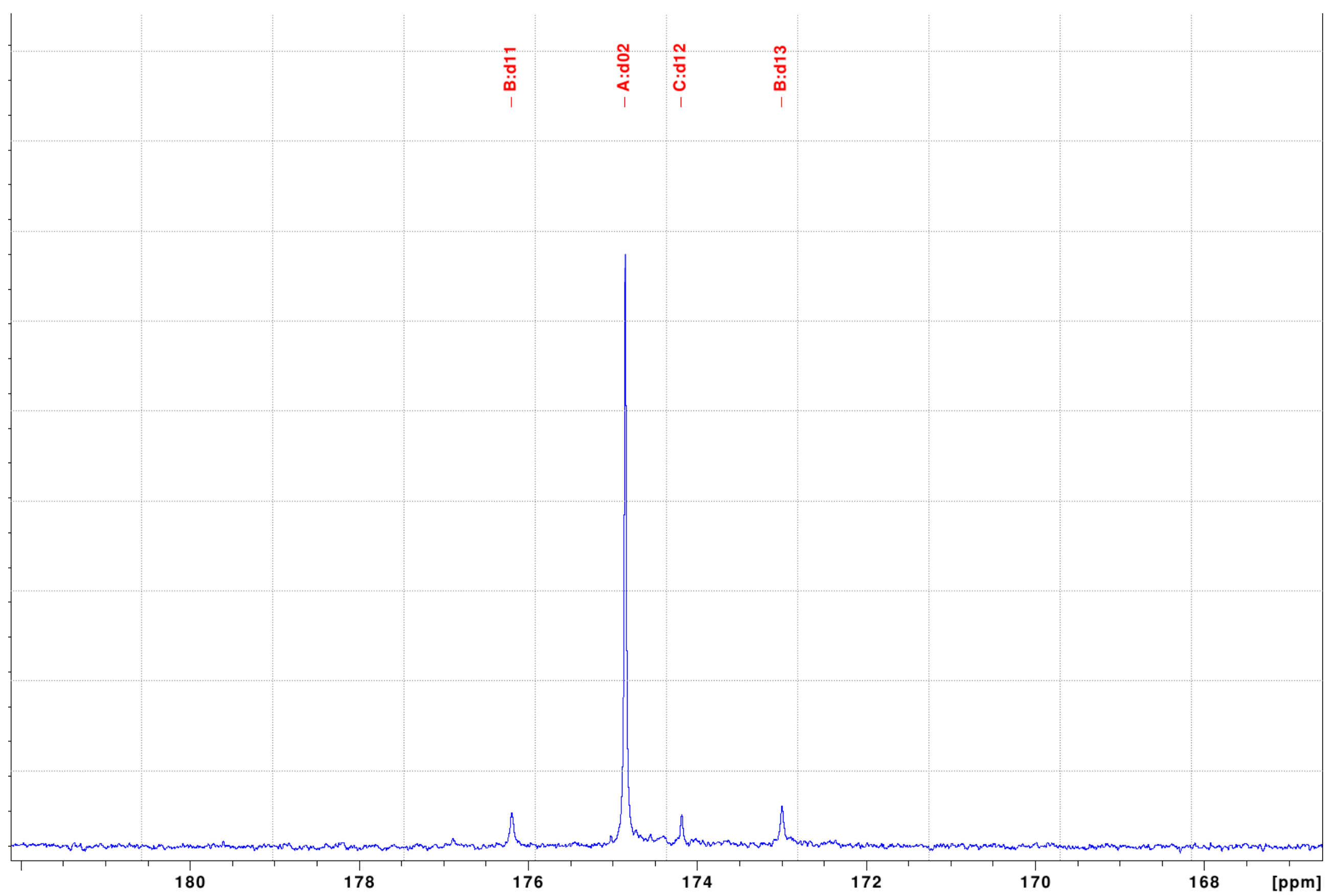




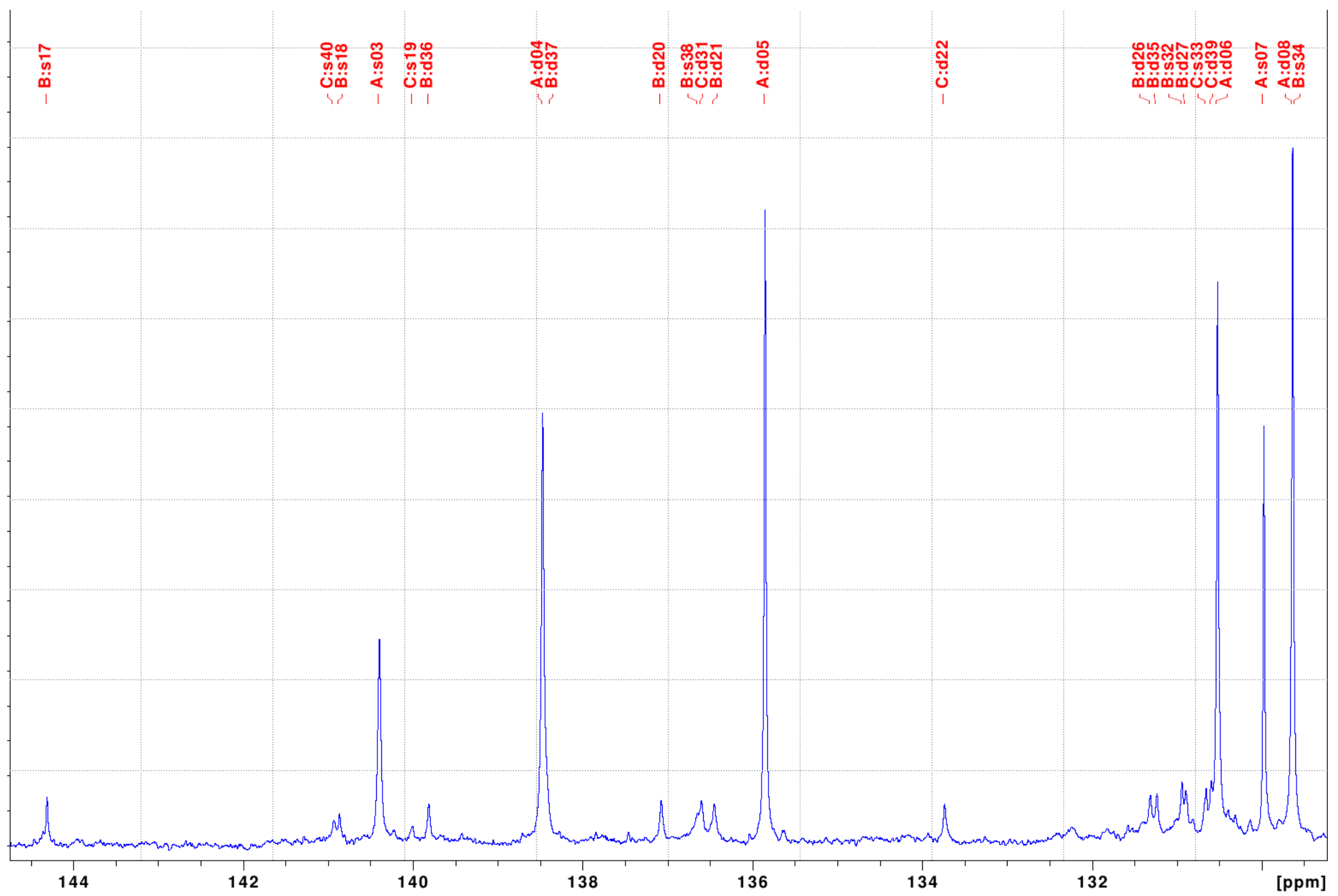




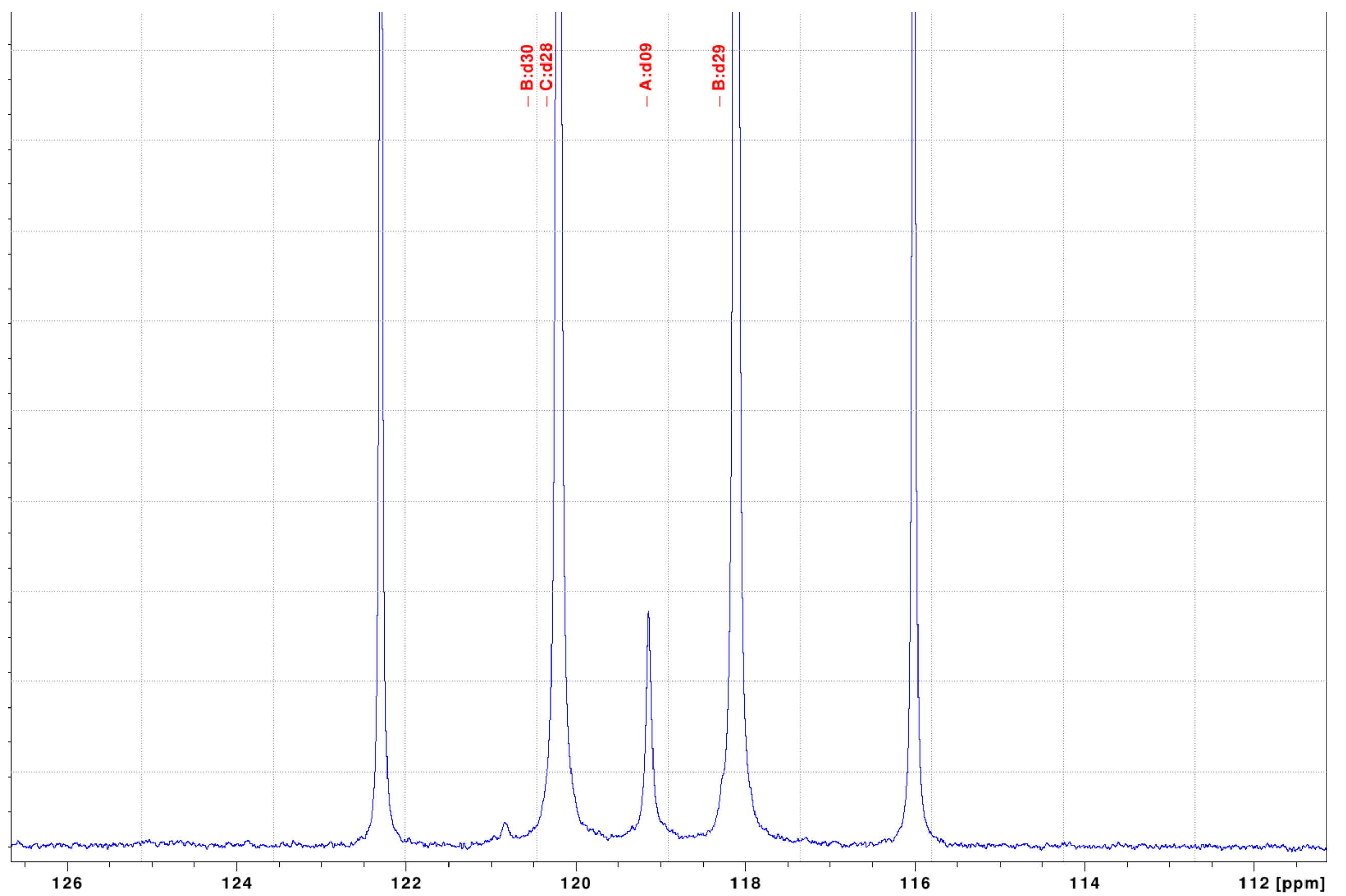




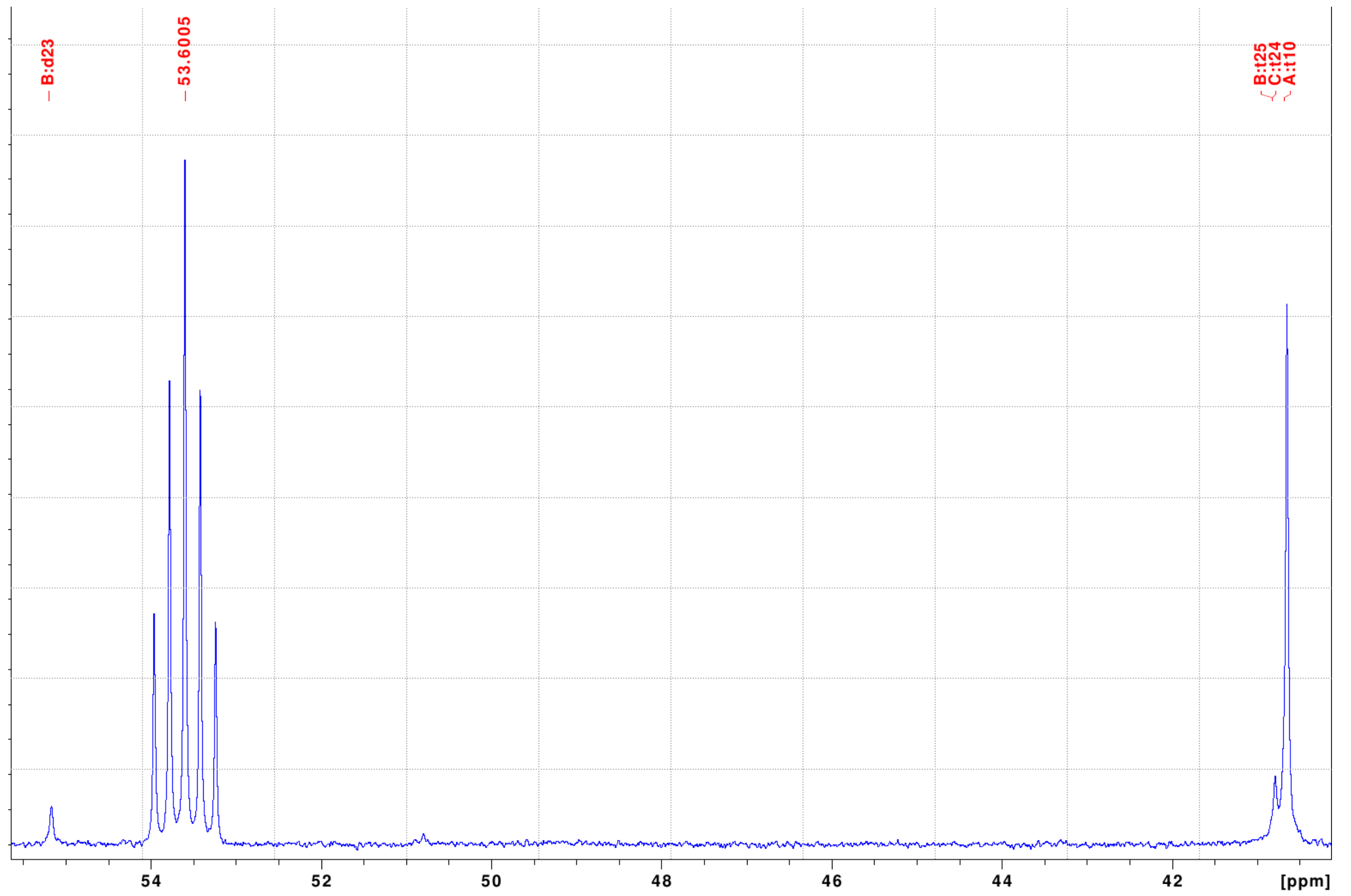


${ }^{1} \mathrm{H}$ NMR spectrum $(600 \mathrm{MHz})$ of the mixture of C1-protonated 2-naphthol and dications 8, 9 in $\mathrm{TfOH}_{-} \mathrm{CD}_{2} \mathrm{Cl}_{2}\left(\mathrm{pages}_{\mathrm{S} 12-\mathrm{S} 16)}\right.$

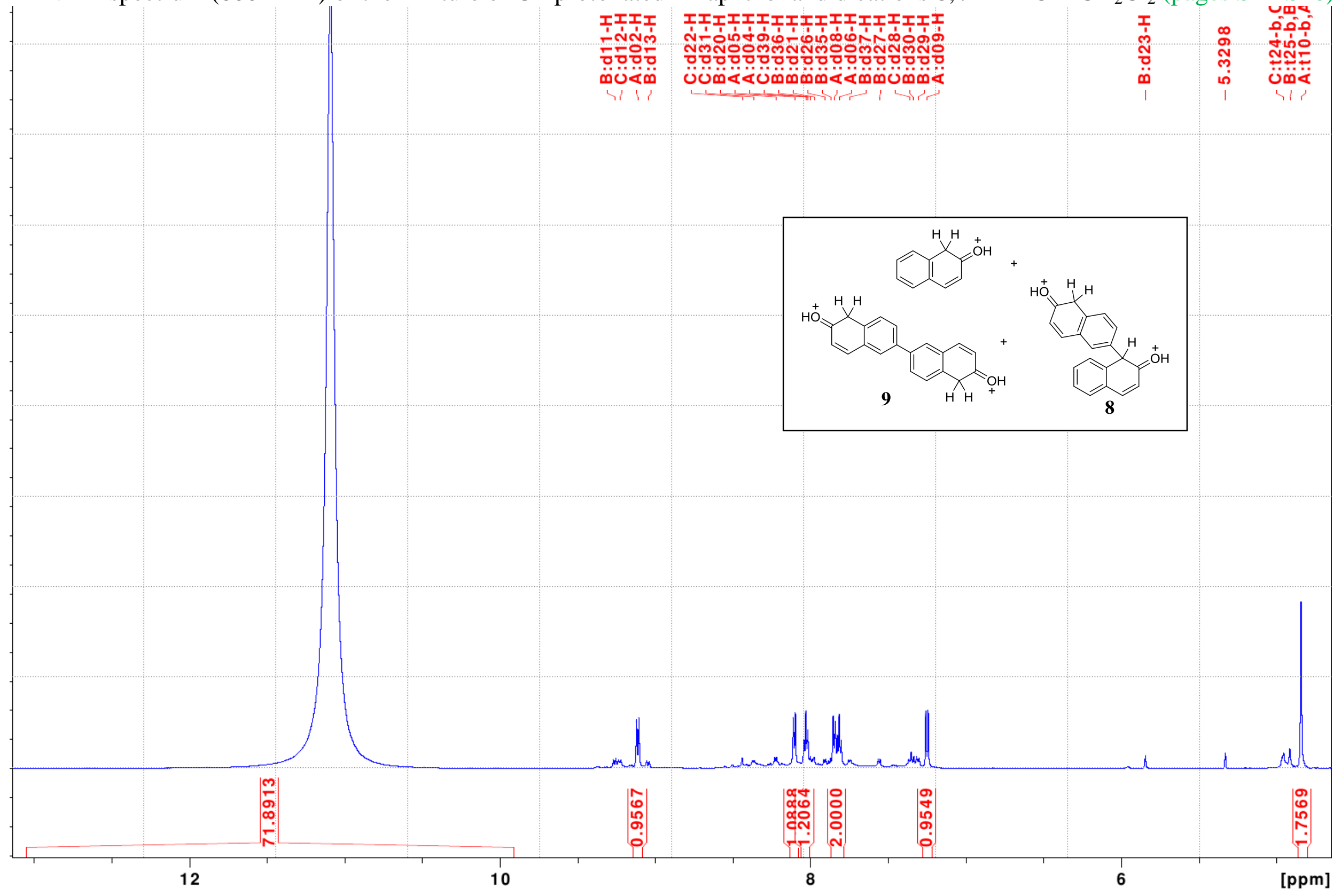




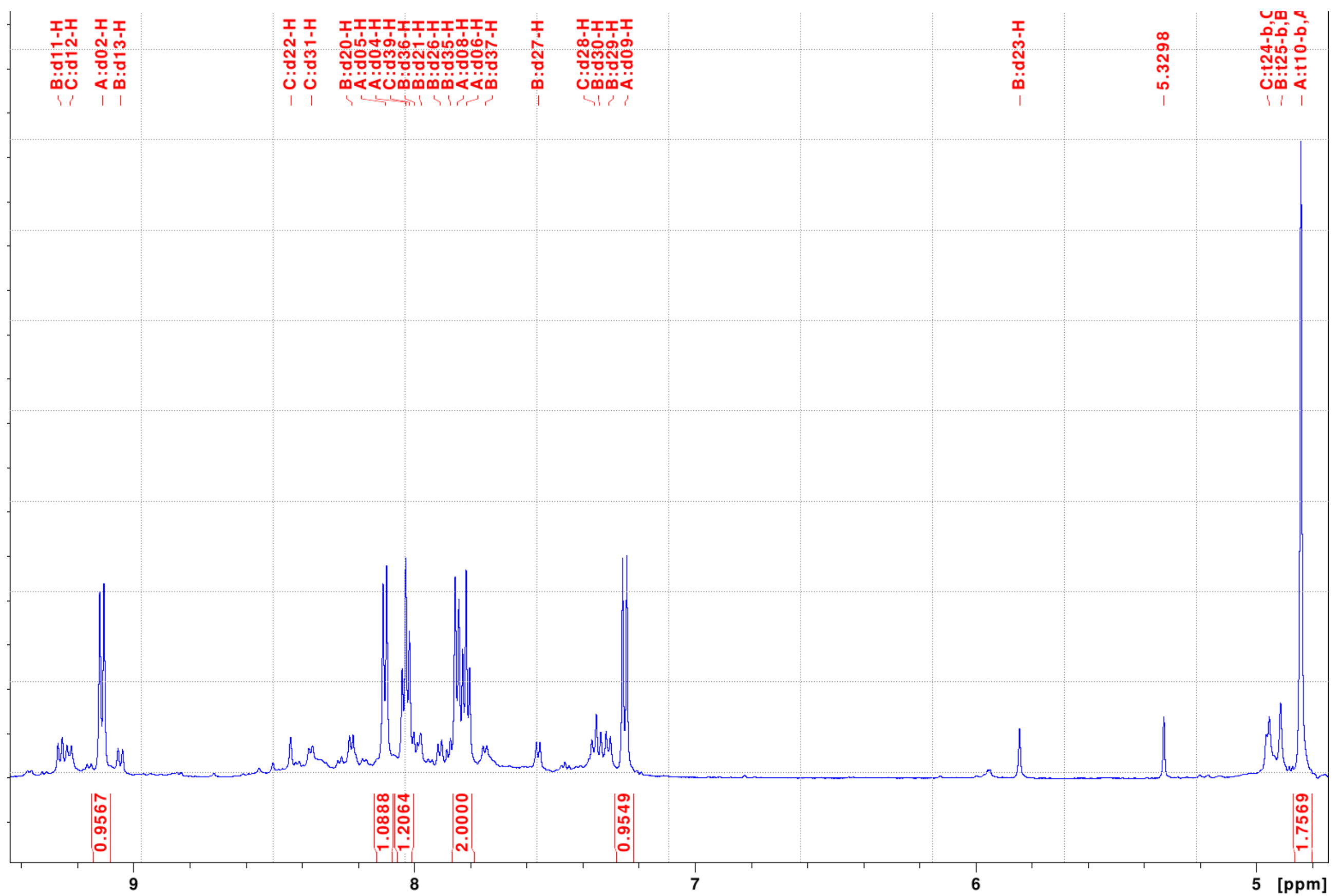




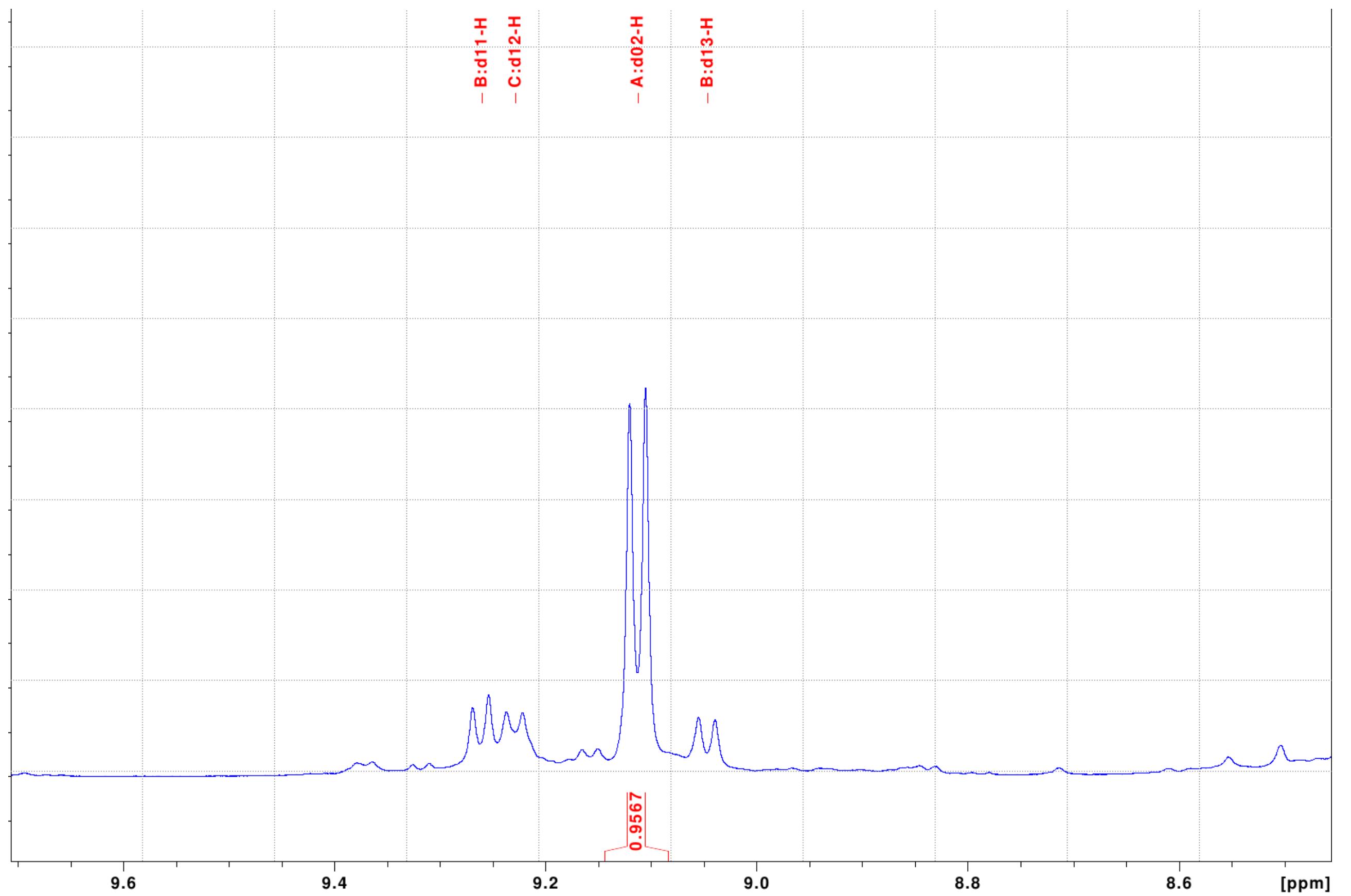




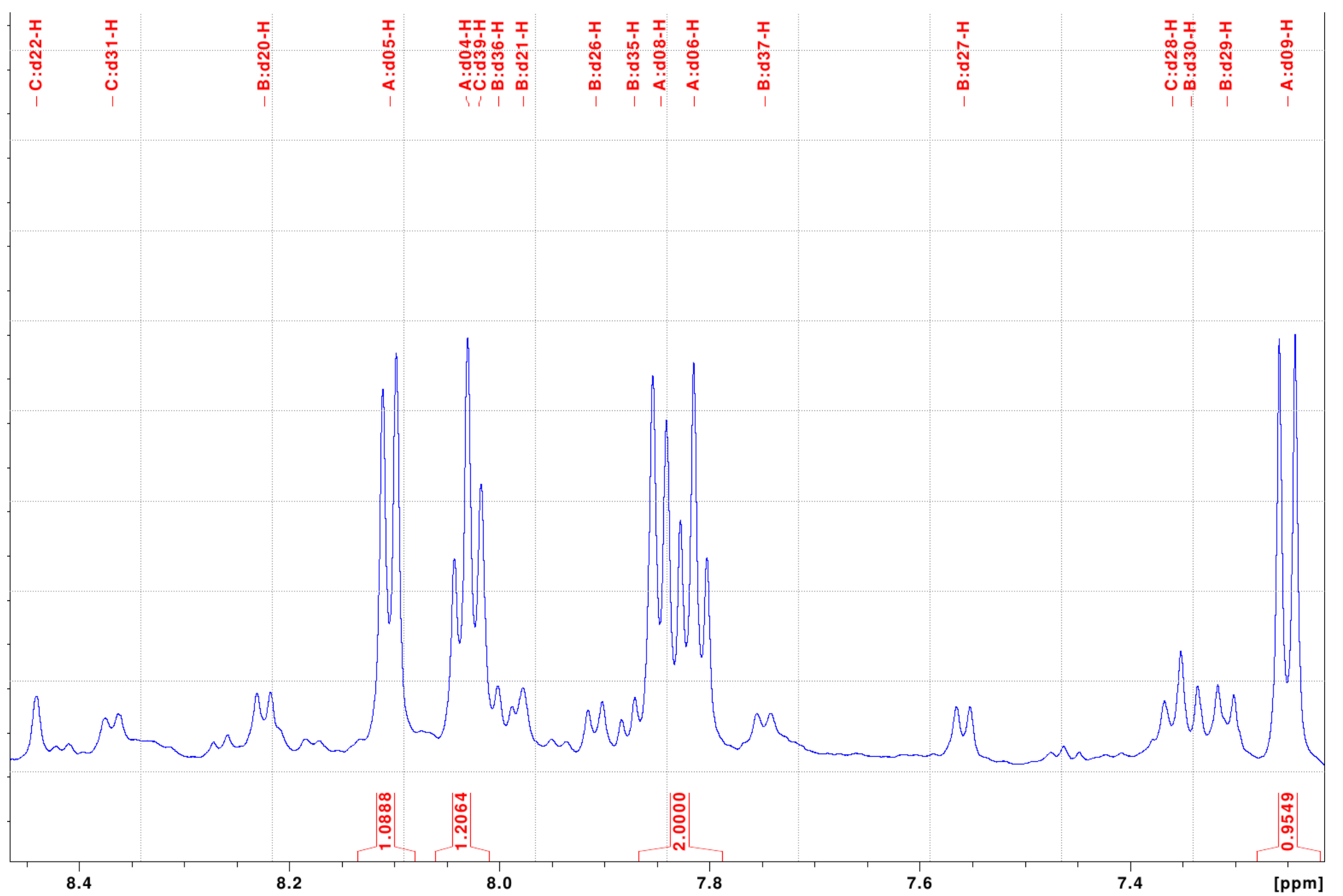




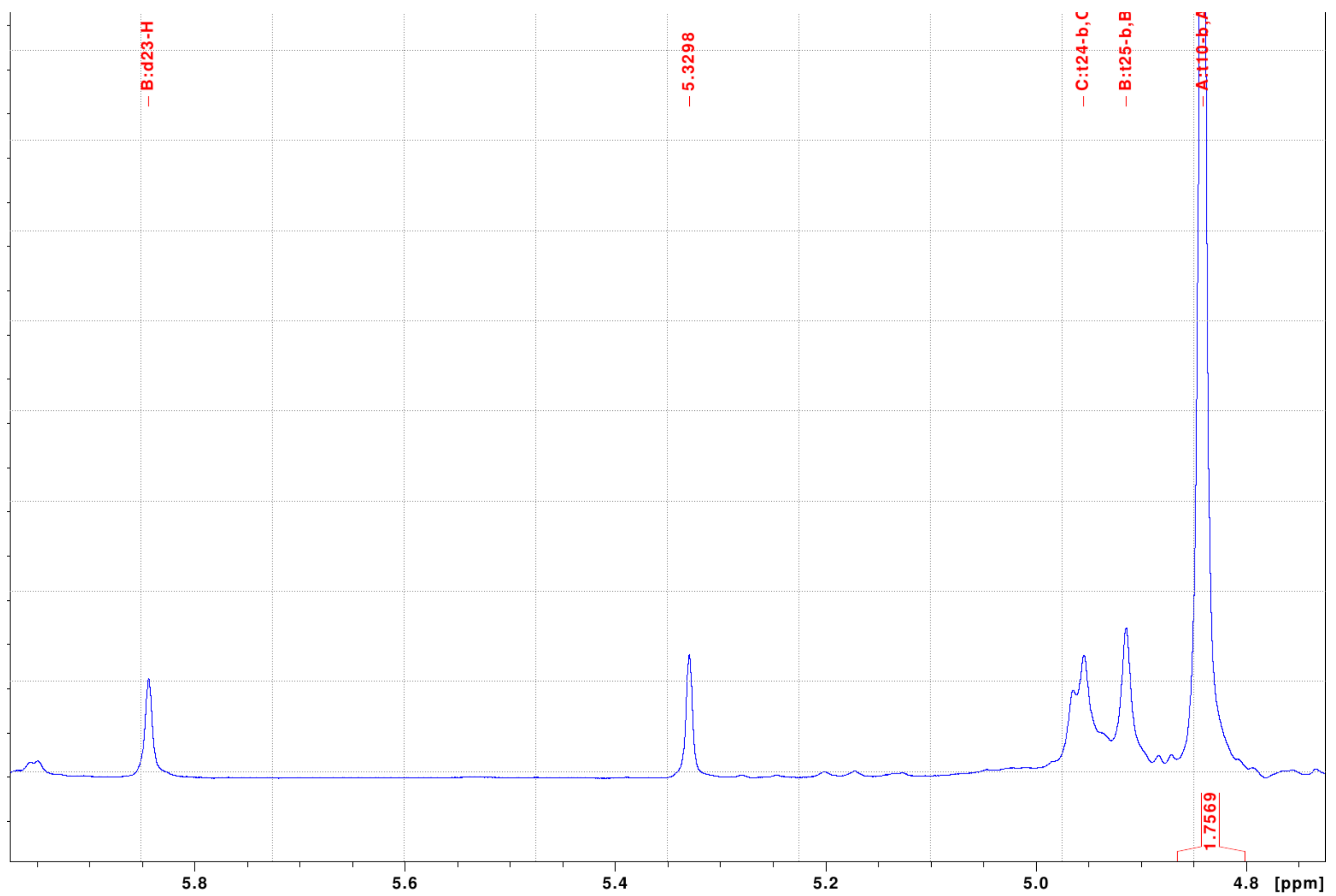




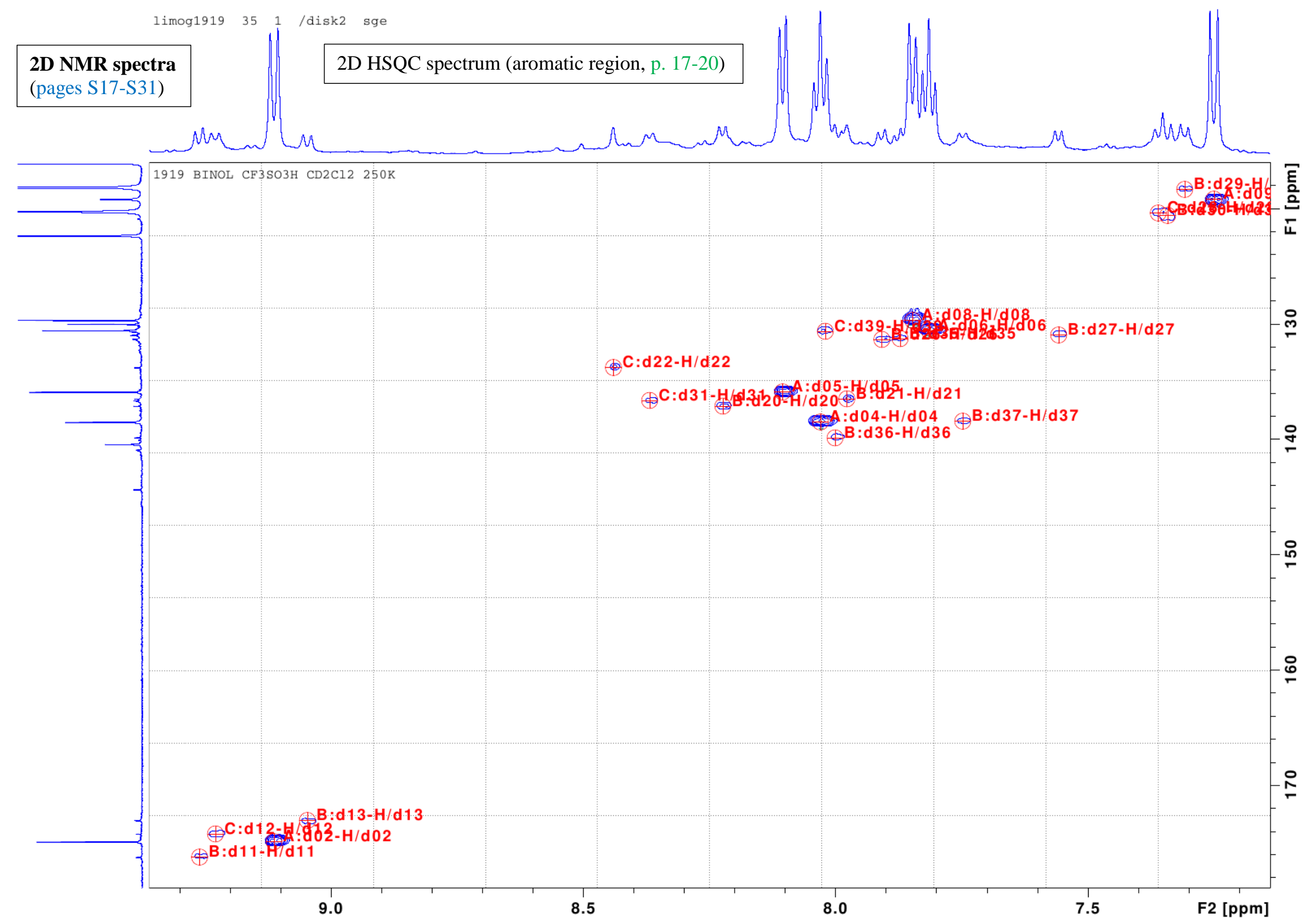


limog1919 351 /disk2 sge

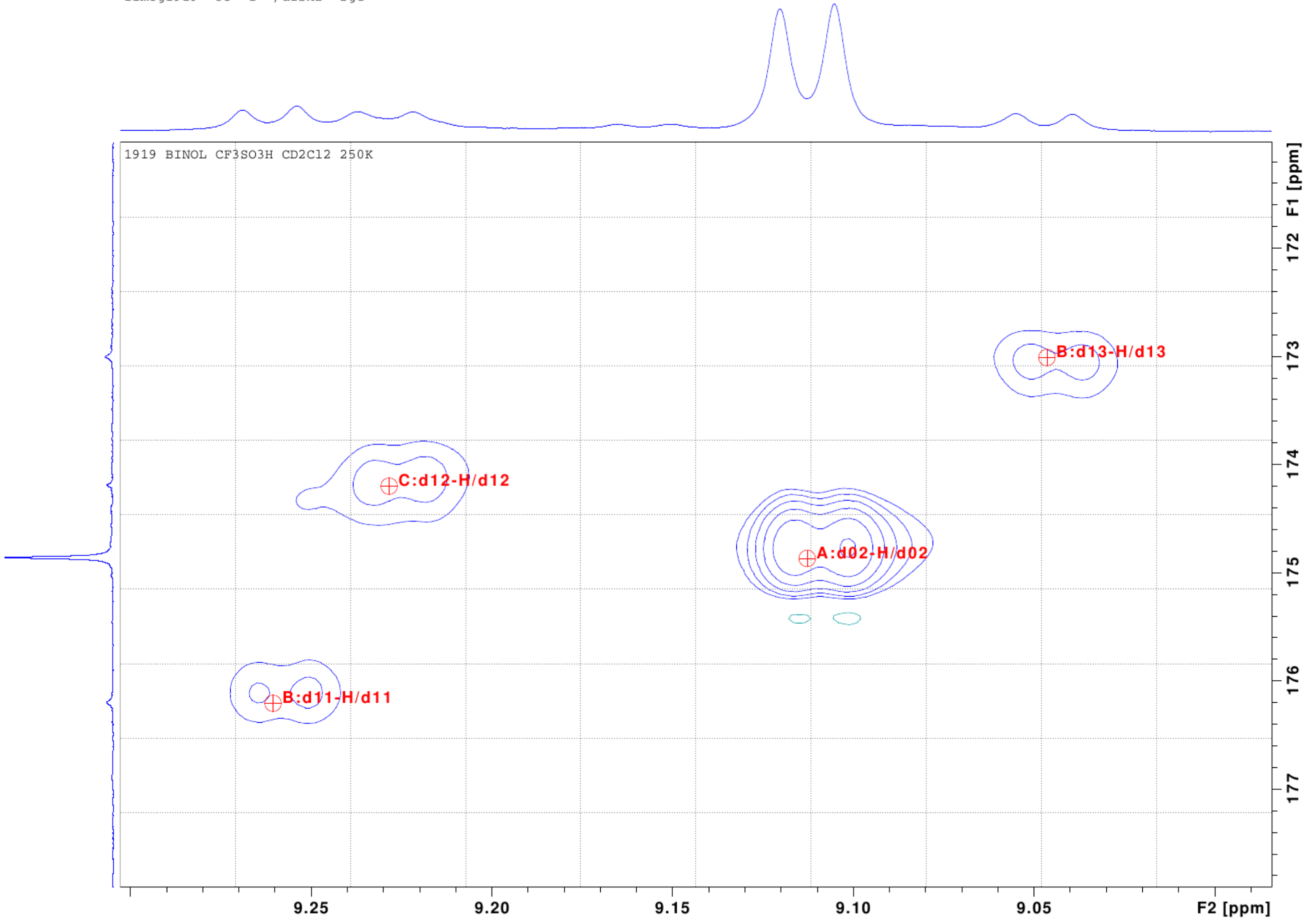




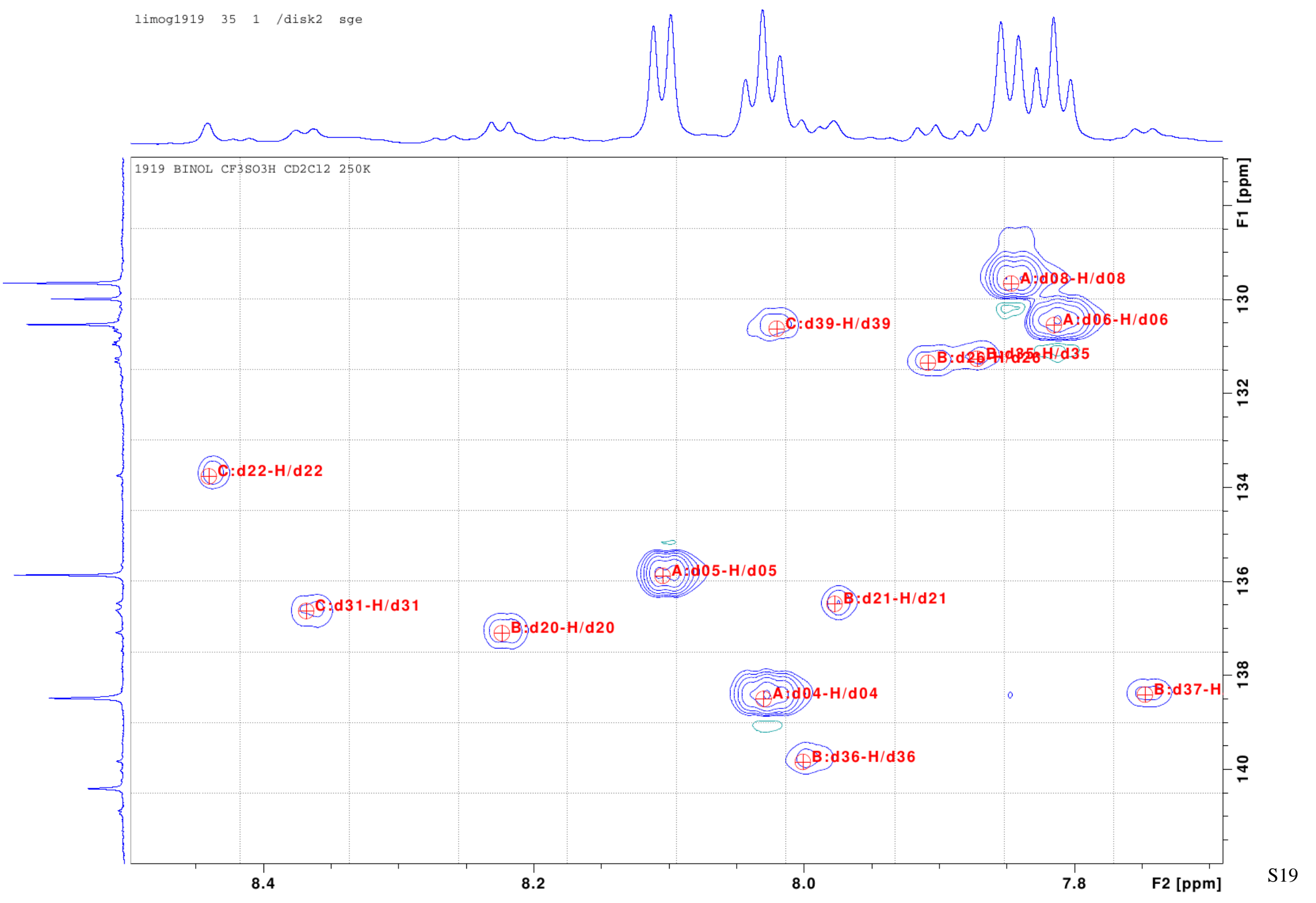




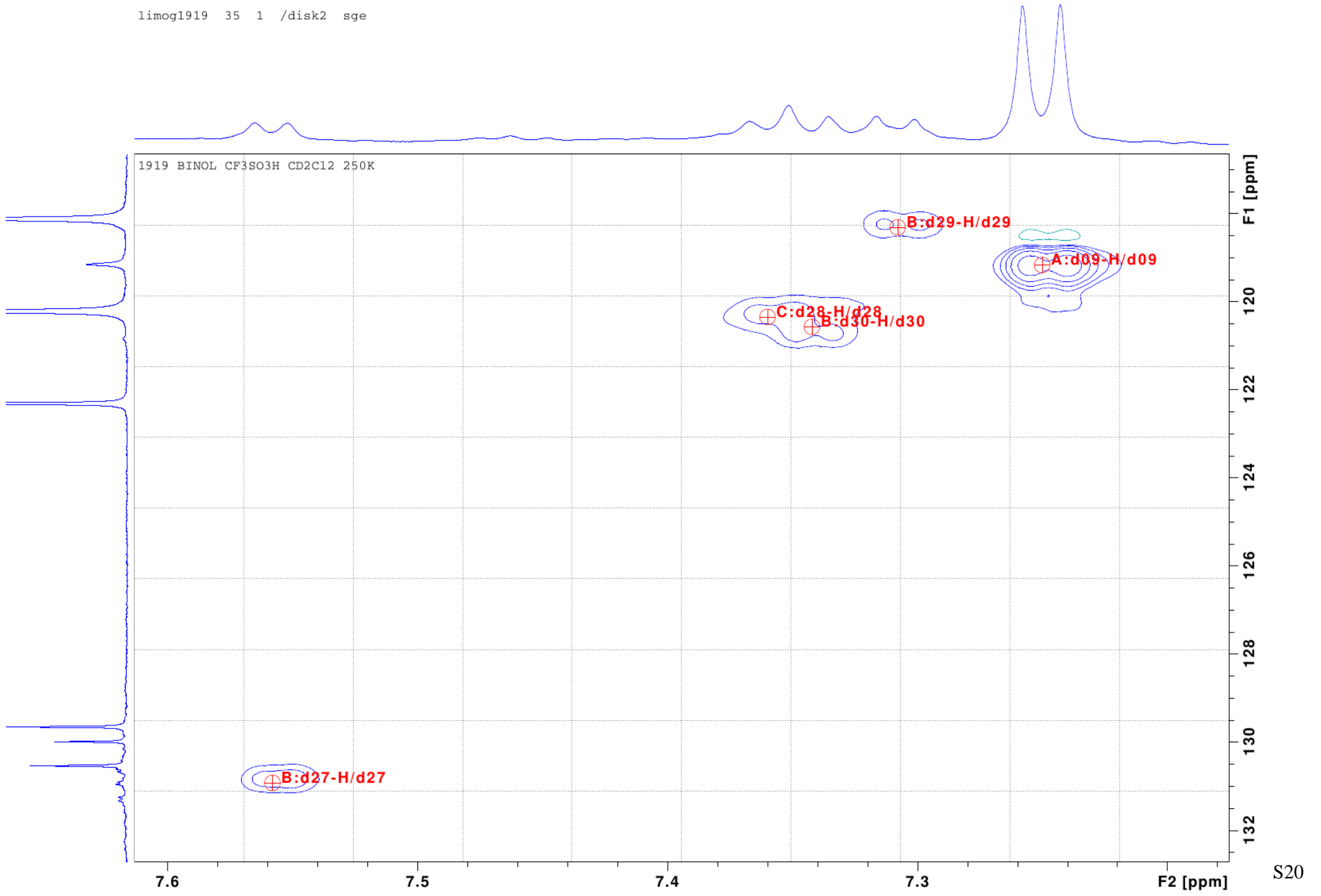




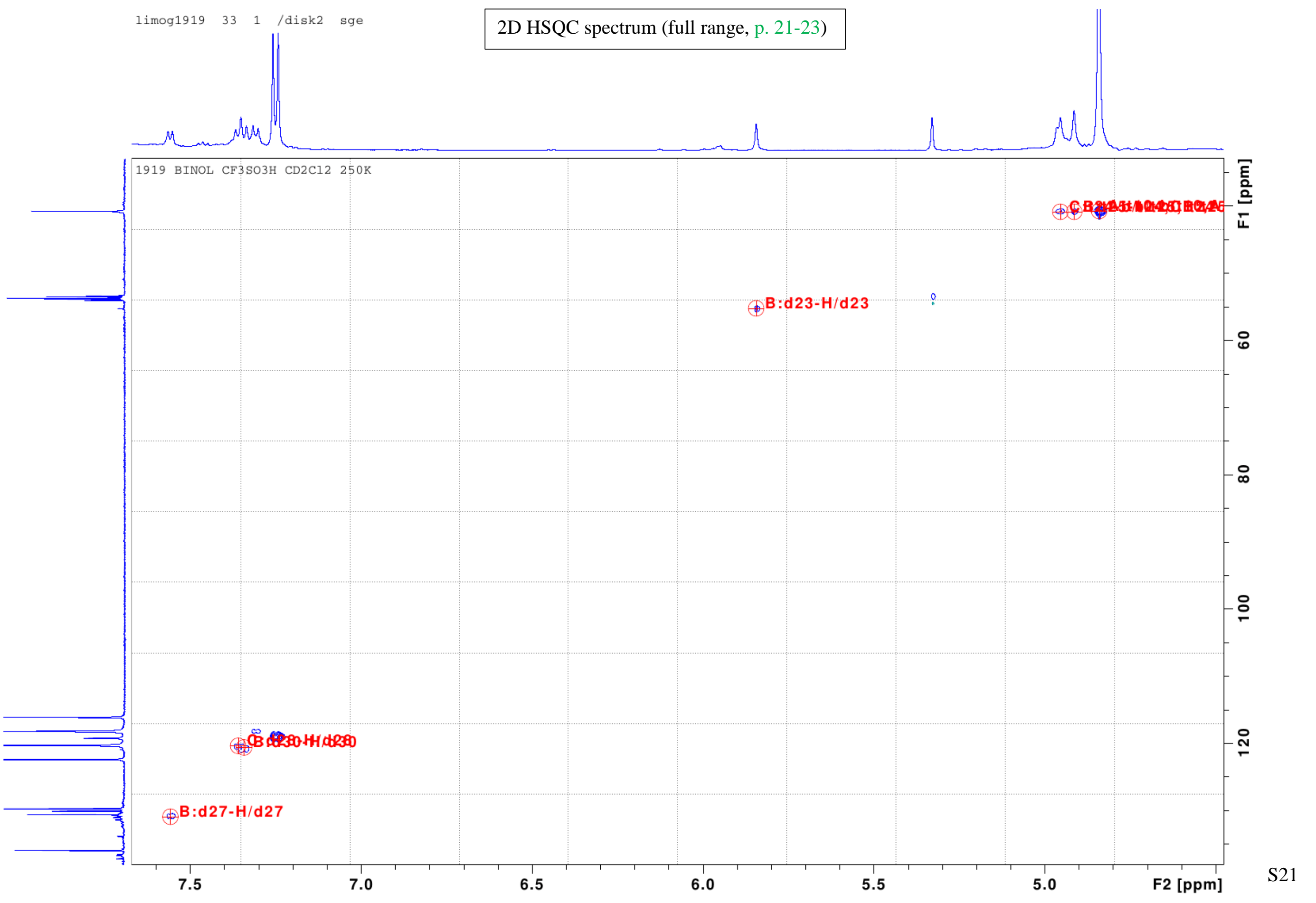




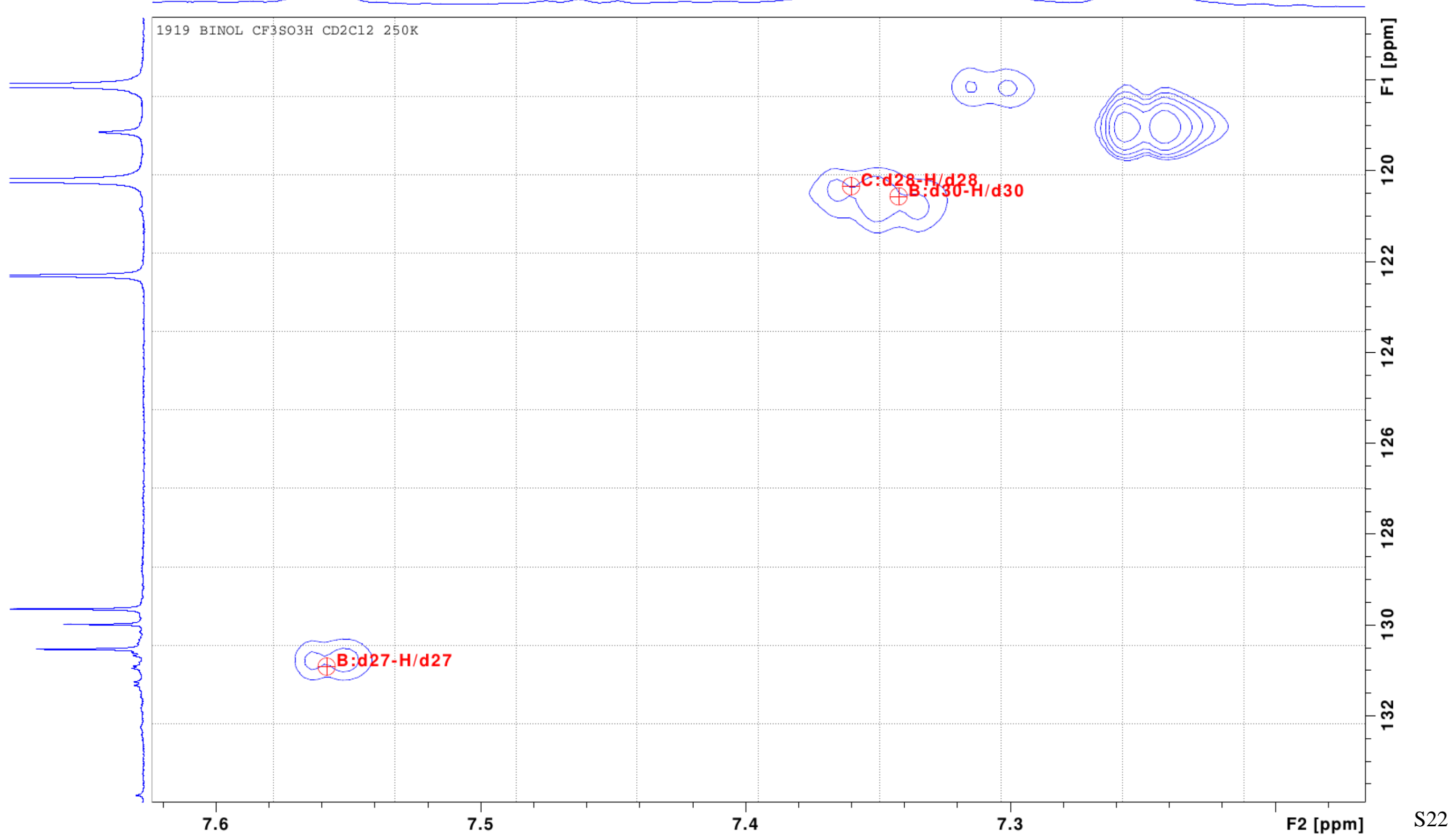




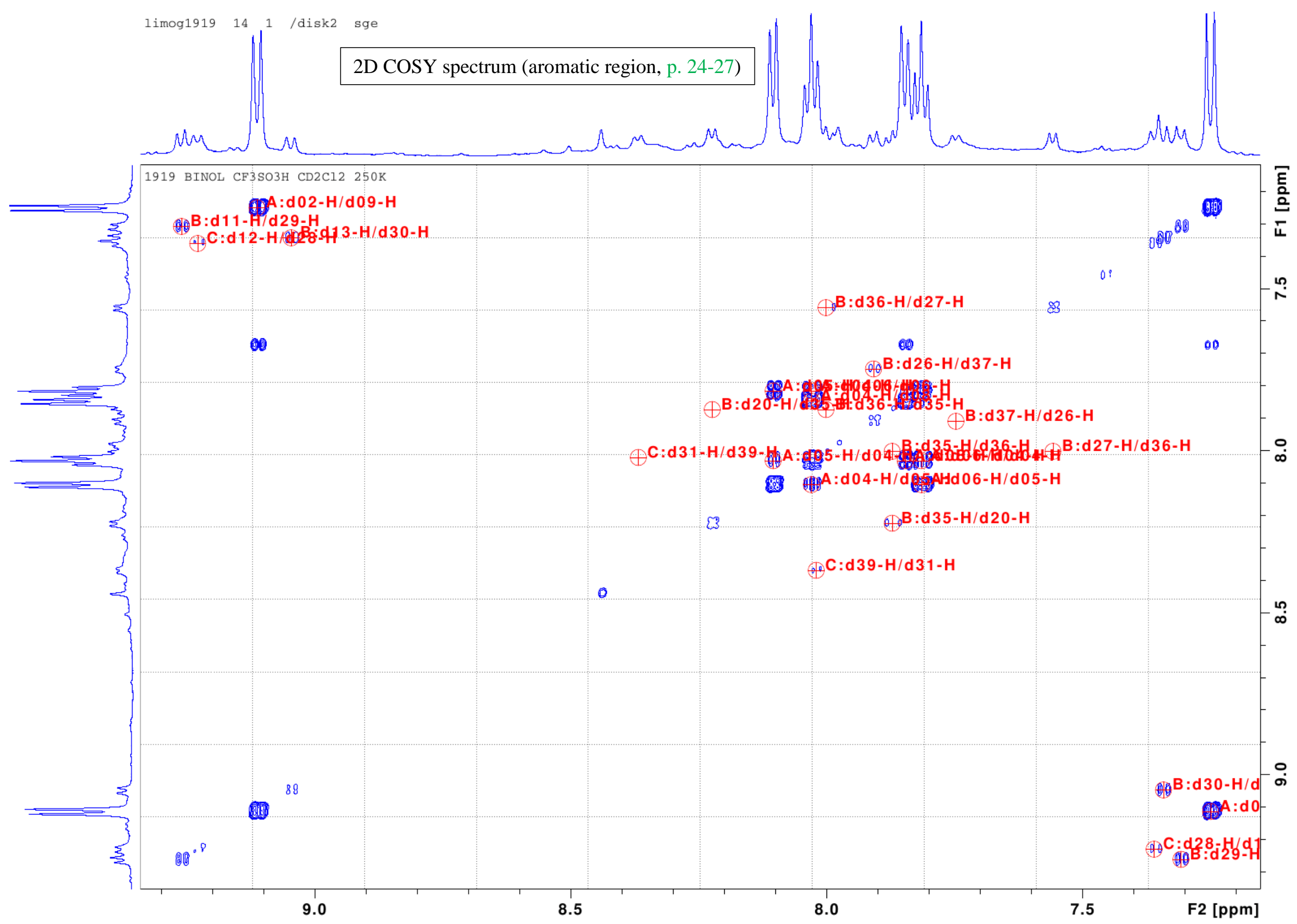




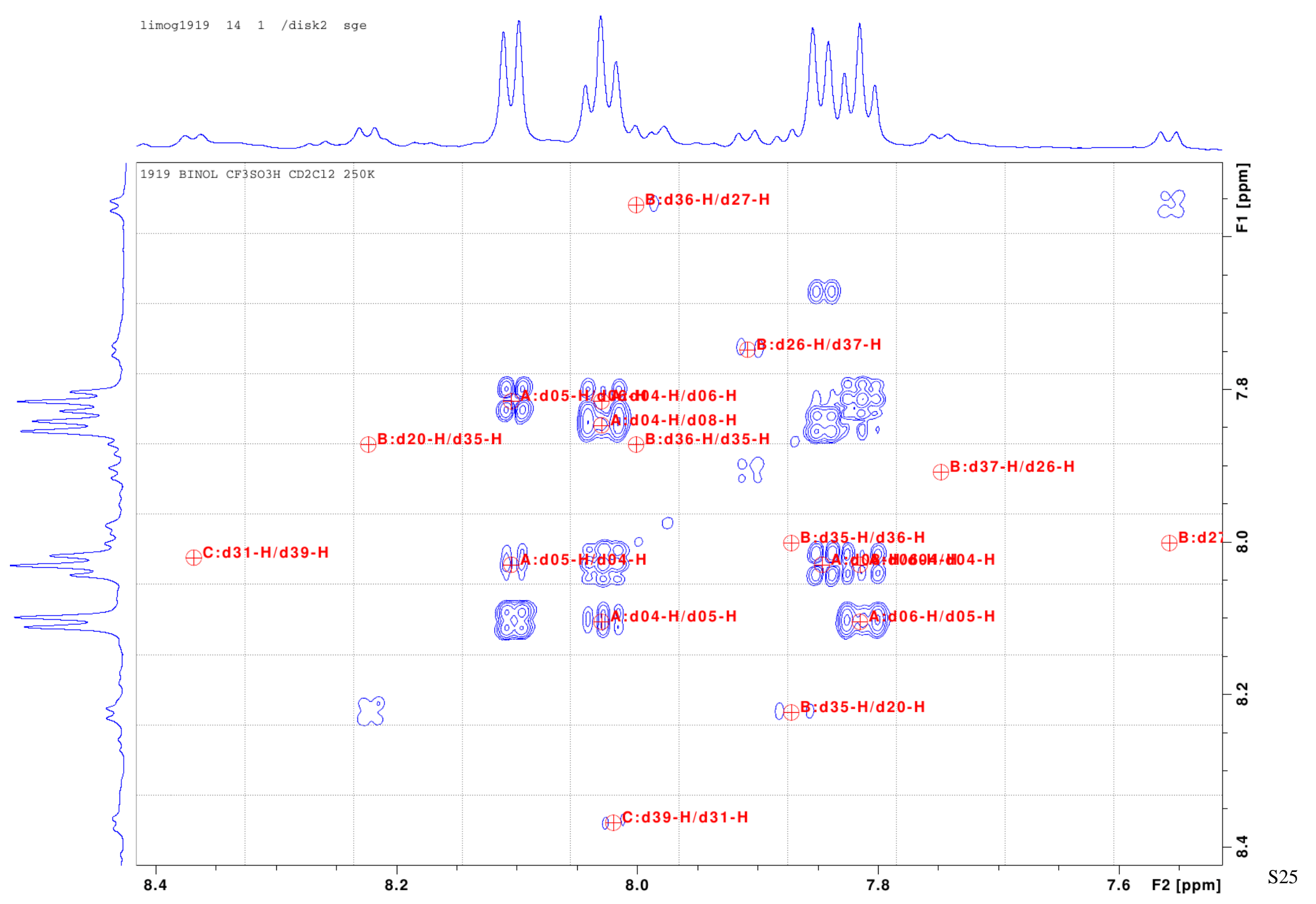




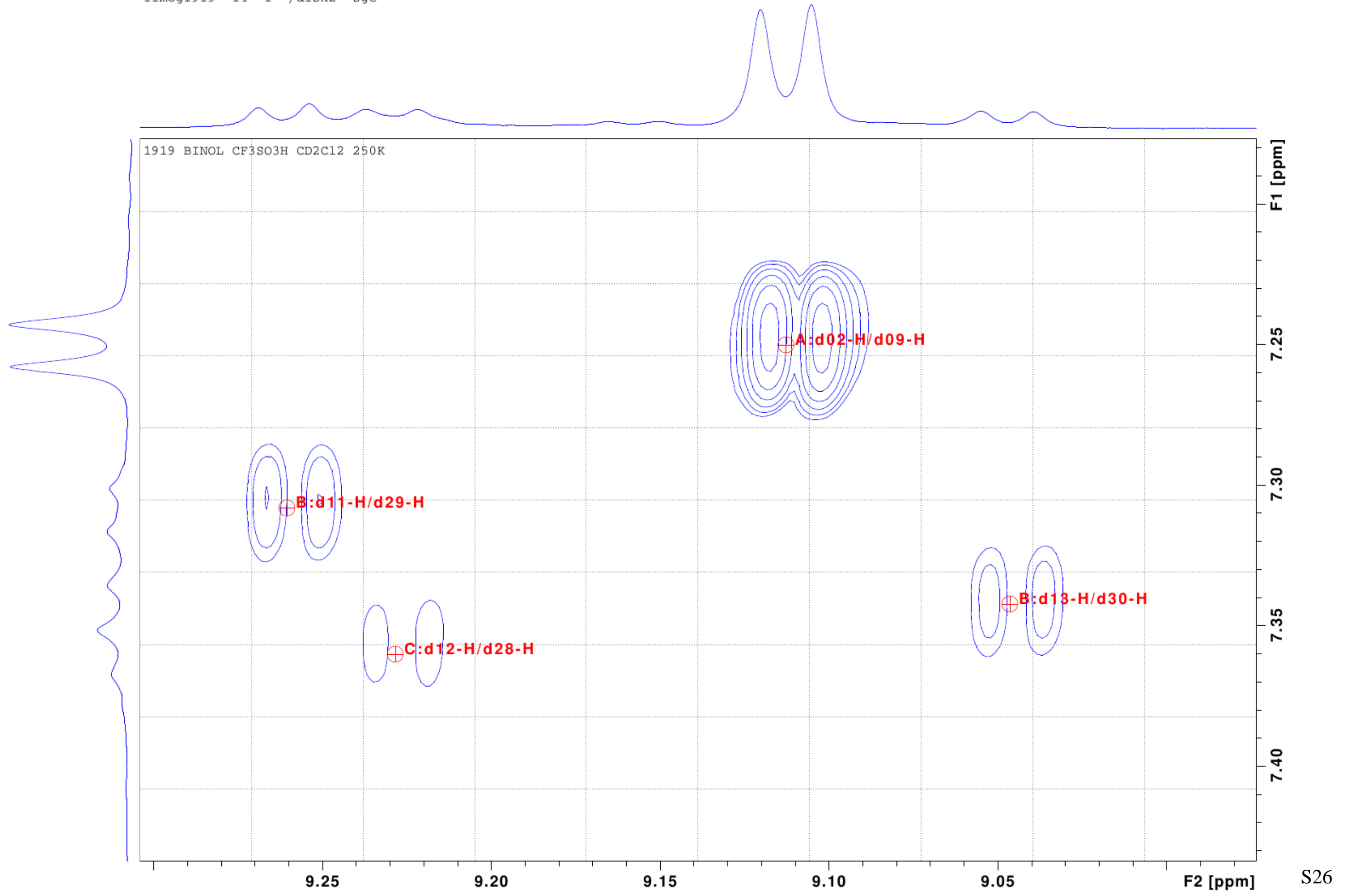




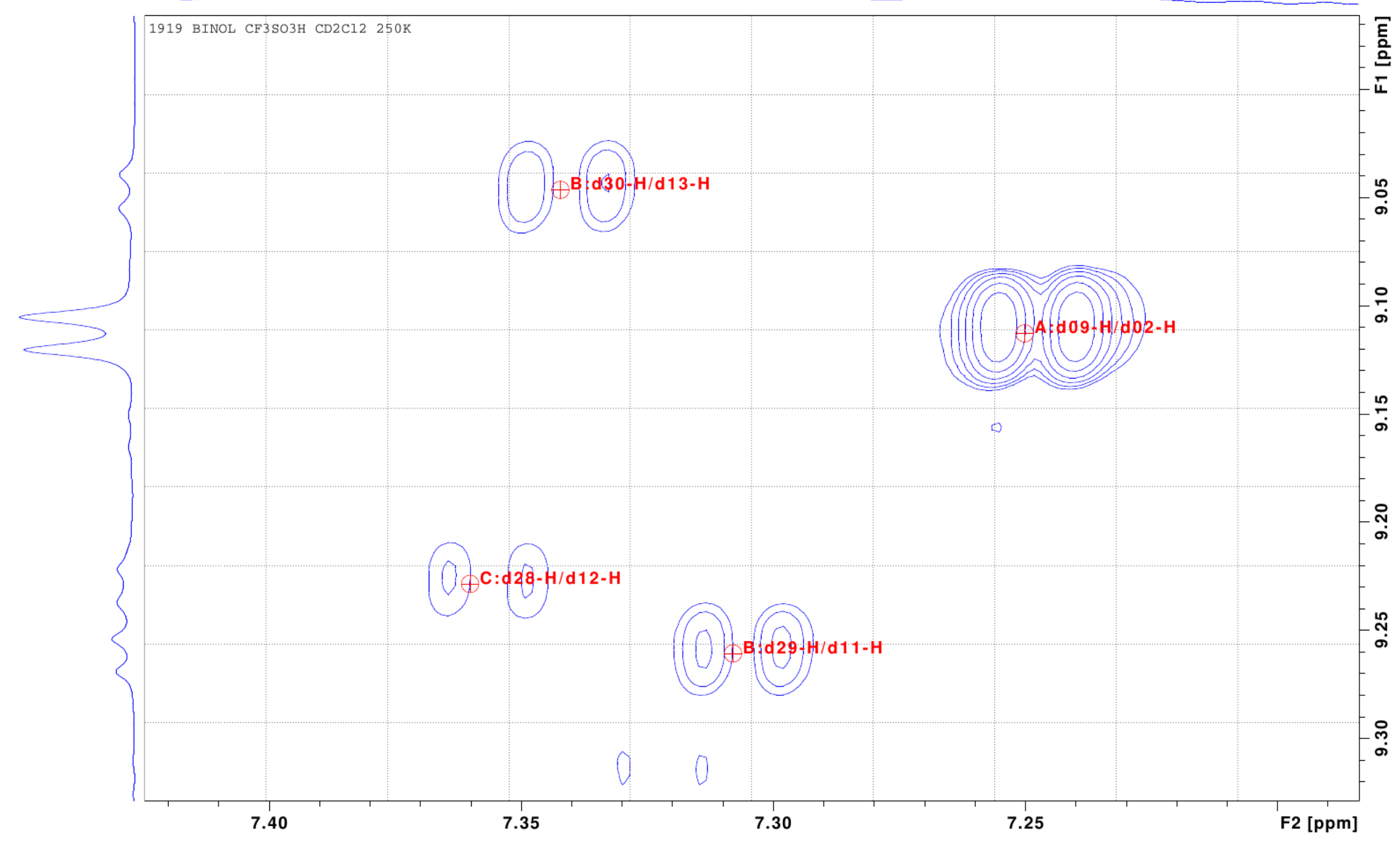




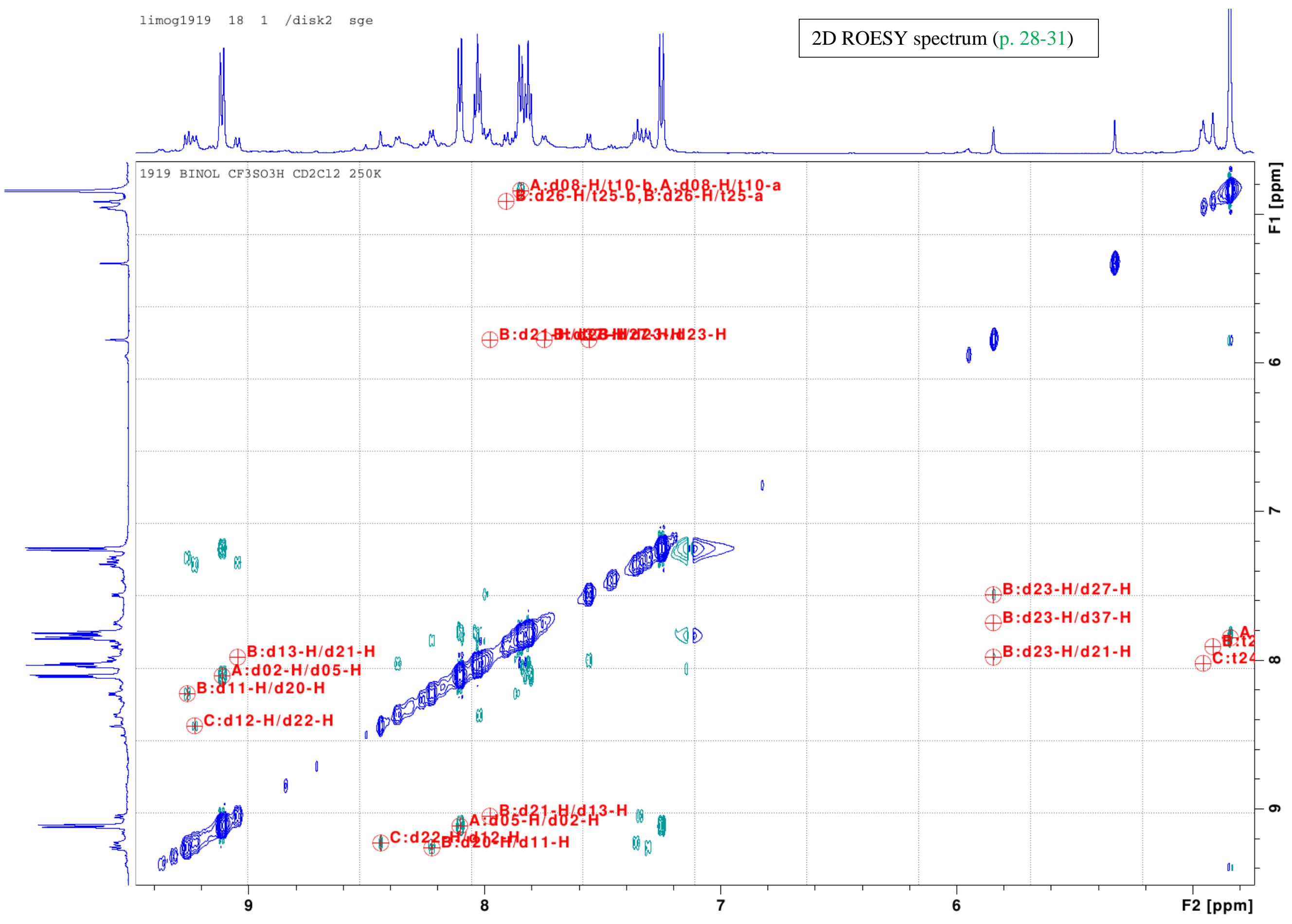


$\operatorname{limog} 1919 \quad 181$ /disk2 sge

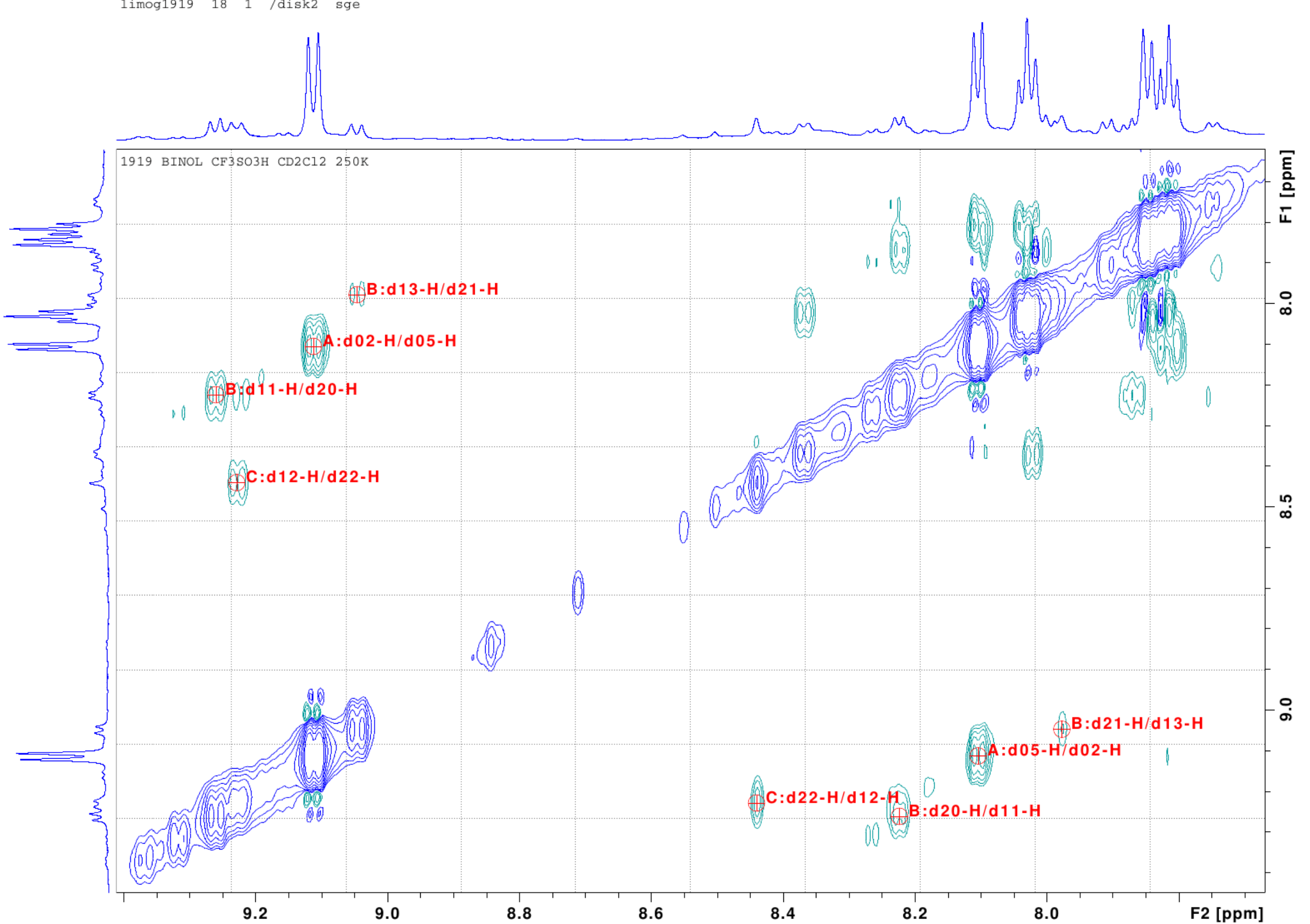




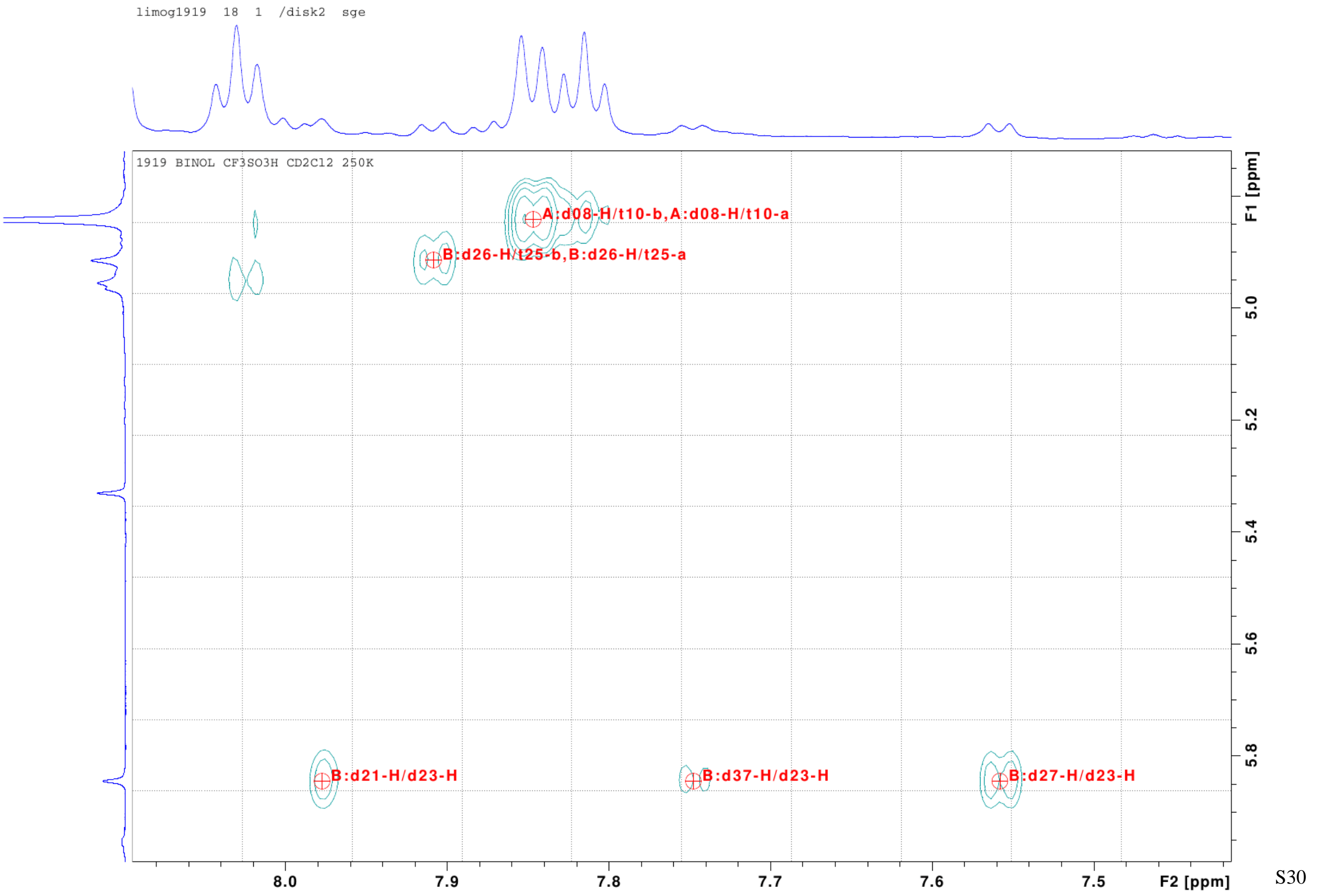


Figure S3. EPR spectra of $\mathbf{1}$ in triflic acid $(0.005 \mathrm{M}$ solution $)$

$-295 \mathrm{~K}$

$-215 \mathrm{~K}$

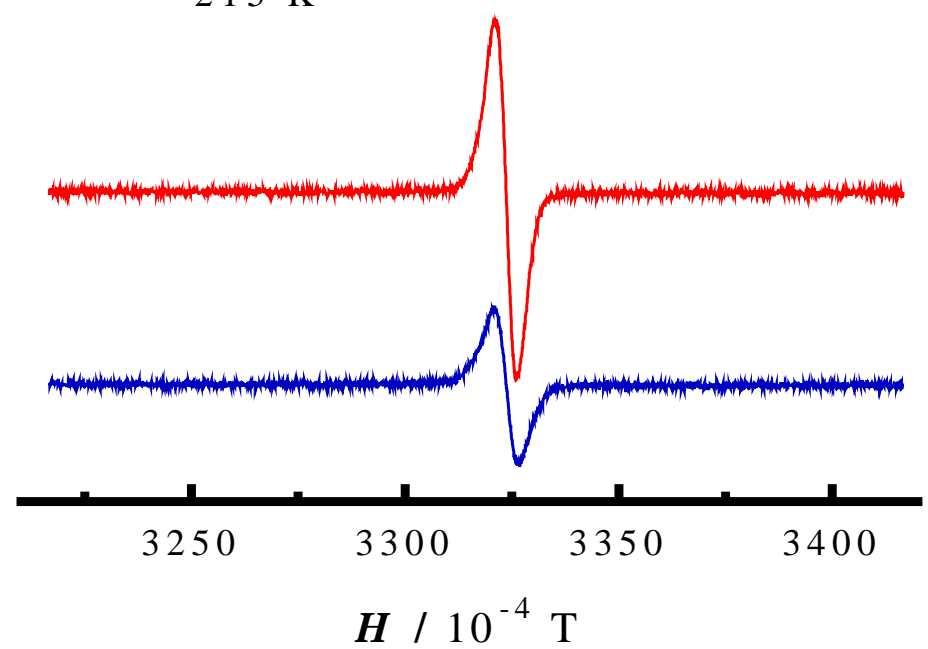

a

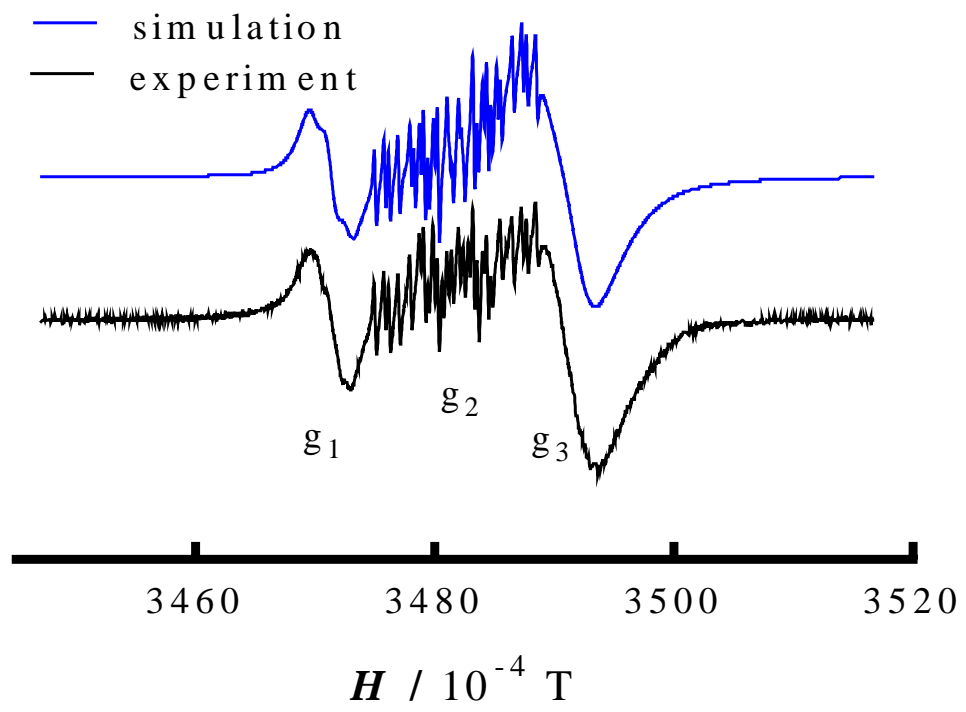

(a) EPR spectra observed at $215<\mathrm{T}<295 \mathrm{~K}$.

(b) EPR spectrum observed after heating at $80{ }^{\circ} \mathrm{C}$ (g-factors, hyperfine structure): $\left(\mathrm{g}_{1}=2.0142\right.$, unresolved hyperfine structure), $\left(\mathrm{g}_{2}=2.0080, a_{\mathrm{H}(1-5)},(\mathrm{G}): 5.82\right.$, $3.27,2.97,1.17,0.79),\left(g_{3}=2.0029\right.$, broadened, no hyperfine structure observed).

\section{DFT $a_{H}(G)$ for radical cation 11 and radical 12}

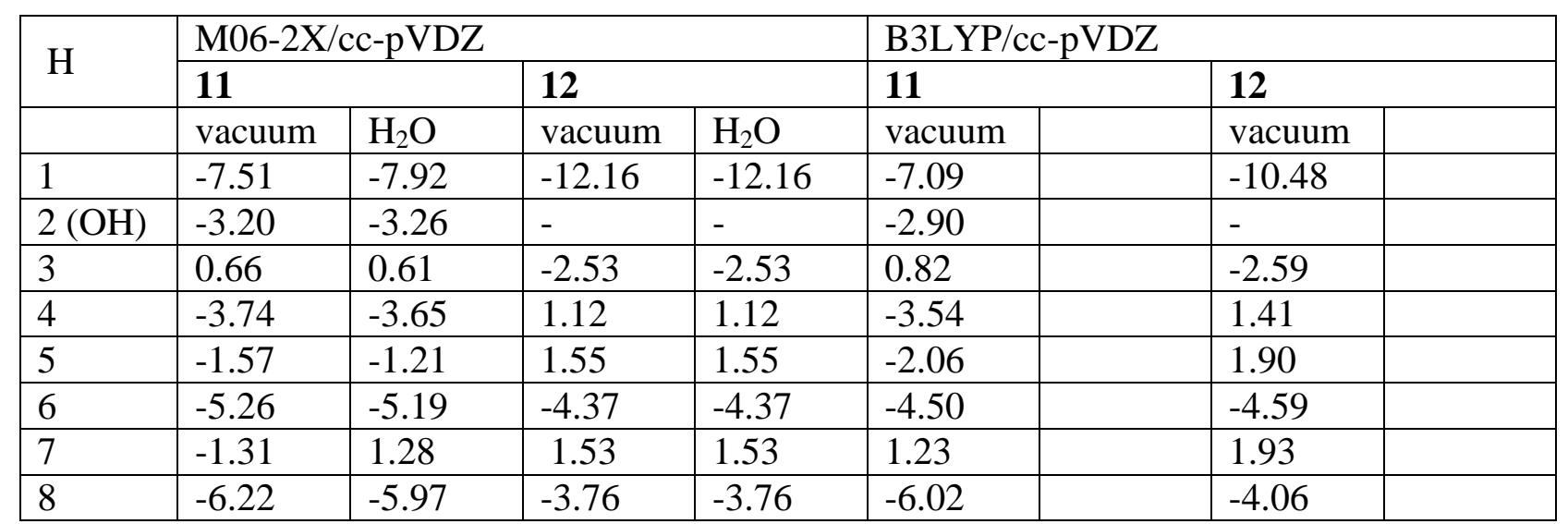

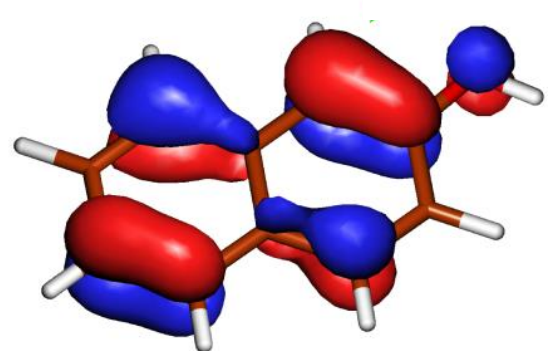

The unpaired electron MO (DFT/B3LYP/cc-pVDZ) in radical cation 11. 
Figure S4. FOD plot for transition state $3 \mathbf{b}^{\neq}$

(FOD plot at $\sigma=0.005$ e/Bohr3 (TPSS/def2-TZVP (T = $5000 \mathrm{~K}$ ) level); FOD depicted in yellow; DFT/PBE/ 11 optimized geometry)

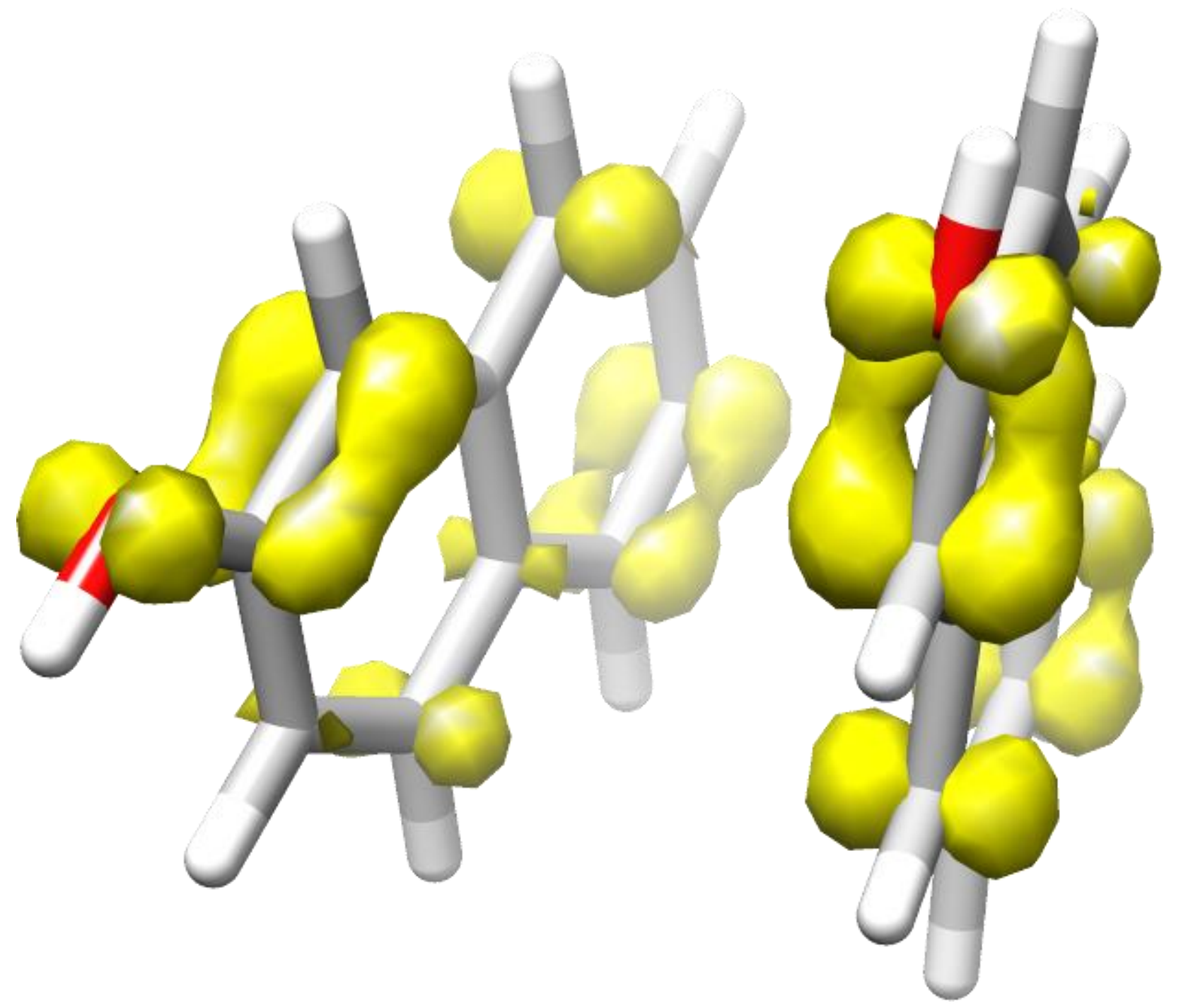




\section{Results of CAS(6X6)/cc-pVDZ calculations of $\mathbf{3 b}$ and $\mathbf{3} \mathbf{b}^{\#}$}

Structure 3b(E=-915.987425 a.u., 0 imaginary frequences $)$

COORDINATES OF ALL ATOMS ARE (ANGS)

ATOM CHARGE $X \quad Y \quad Z$

\begin{tabular}{lrrrr}
---1 & \multicolumn{1}{c}{-1.0} & -0.6131748561 & -0.5102411352 & 1.2475839892 \\
$\mathrm{C} 1$ & 6.0 & 0.6131748561 & 0.5102411352 & 1.2475839892 \\
$\mathrm{C} 2$ & 6.0 & -1.8841984788 & 0.1091747482 & 1.7600794271 \\
$\mathrm{C} 2$ & 6.0 & 1.8841984788 & -0.1091747482 & 1.7600794271 \\
$\mathrm{C} 3$ & 6.0 & -3.1250243329 & -0.2574663940 & 1.2849862562 \\
$\mathrm{C} 3$ & 6.0 & 3.1250243329 & 0.2574663940 & 1.2849862562 \\
$\mathrm{C} 4$ & 6.0 & -3.1932430816 & -1.1400821327 & 0.2201872800 \\
$\mathrm{C} 4$ & 6.0 & 3.1932430816 & 1.1400821327 & 0.2201872800 \\
$\mathrm{C} 4 \mathrm{~A}$ & 6.0 & -2.0635293526 & -1.6930503556 & -0.4383157158 \\
$\mathrm{C} 4 \mathrm{~A}$ & 6.0 & 2.0635293526 & 1.6930503556 & -0.4383157158 \\
$\mathrm{C} 5$ & 6.0 & -2.2405688277 & -2.5686197748 & -1.5468852751 \\
$\mathrm{C} 5$ & 6.0 & 2.2405688277 & 2.5686197748 & -1.5468852751 \\
$\mathrm{C} 6$ & 6.0 & -1.1455662561 & -3.0994128910 & -2.1840314267 \\
$\mathrm{C} 6$ & 6.0 & 1.1455662561 & 3.0994128910 & -2.1840314267 \\
$\mathrm{C} 7$ & 6.0 & 0.1421729910 & -2.7622839928 & -1.7211713633 \\
$\mathrm{C} 7$ & 6.0 & -0.1421729910 & 2.7622839928 & -1.7211713633 \\
$\mathrm{C} 8$ & 6.0 & 0.3239191789 & -1.9141054833 & -0.6491678230 \\
$\mathrm{C} 8$ & 6.0 & -0.3239191789 & 1.9141054833 & -0.6491678230 \\
$\mathrm{C} 8 \mathrm{~A}$ & 6.0 & -0.7788944550 & -1.3640431702 & 0.0074541768 \\
$\mathrm{C} 8 \mathrm{~A}$ & 6.0 & 0.7788944550 & 1.3640431702 & 0.0074541768 \\
$\mathrm{O}$ & 8.0 & -1.7062488855 & 0.9412204390 & 2.7343541775 \\
$\mathrm{O}$ & 8.0 & 1.7062488855 & -0.9412204390 & 2.7343541775 \\
$\mathrm{H} 3$ & 1.0 & -4.0257618902 & 0.1394450036 & 1.7311817150 \\
$\mathrm{H} 3$ & 1.0 & 4.0257618902 & -0.1394450036 & 1.7311817150 \\
$\mathrm{H} 4$ & 1.0 & -4.1758235036 & -1.4190038529 & -0.1412181502 \\
$\mathrm{H} 4$ & 1.0 & 4.1758235036 & 1.4190038529 & -0.1412181502 \\
$\mathrm{H} 5$ & 1.0 & -3.2410513123 & -2.8131186620 & -1.8755125437 \\
$\mathrm{H} 5$ & 1.0 & 3.2410513123 & 2.8131186620 & -1.8755125437 \\
$\mathrm{H} 6$ & 1.0 & -1.2618848187 & -3.7681751267 & -3.0236434234 \\
$\mathrm{H} 6$ & 1.0 & 1.2618848187 & 3.7681751267 & -3.0236434234 \\
$\mathrm{H} 7$ & 1.0 & 1.0081570097 & -3.1807230094 & -2.2160659698 \\
$\mathrm{H} 7$ & 1.0 & -1.0081570097 & 3.1807230094 & -2.2160659698 \\
$\mathrm{H} 8$ & 1.0 & 1.3315500786 & -1.6918022179 & -0.3311941222 \\
$\mathrm{H} 8$ & 1.0 & -1.3315500786 & 1.6918022179 & -0.3311941222 \\
$\mathrm{H} 1$ & 1.0 & -0.4042186787 & -1.2197925766 & 2.0594349097 \\
$\mathrm{H} 1$ & 1.0 & 0.4042186787 & 1.2197925766 & 2.0594349097 \\
$\mathrm{HOH}$ & 1.0 & -2.5153449936 & 1.2602399577 & 3.1272486366 \\
$\mathrm{HOH}$ & 2.5153449936 & -1.2602399577 & 3.1272486366
\end{tabular}


Structure $\mathbf{3 b}^{\neq}(\mathrm{E}=-915.942858$ a.u., 1 imaginary frequence, 154.6i)

COORDINATES OF ALL ATOMS ARE (ANGS)
ATOM
CHARGE
Y
Z

$\begin{array}{lrrrr}\text { C1 } & 6.0 & -0.6100561994 & -1.2037555162 & 1.5557572443 \\ \text { C1 } & 6.0 & 0.6100561994 & 1.2037555162 & 1.5557572443 \\ \text { C2 } & 6.0 & -1.7740308505 & -0.5260071131 & 2.0628246037 \\ \text { C2 } & 6.0 & 1.7740308505 & 0.5260071131 & 2.0628246037 \\ \text { C3 } & 6.0 & -2.8094053452 & -0.1276553966 & 1.1956123909 \\ \text { C3 } & 6.0 & 2.8094053452 & 0.1276553966 & 1.1956123909 \\ \text { C4 } & 6.0 & -2.7212694961 & -0.4564889697 & -0.1260828618 \\ \text { C4 } & 6.0 & 2.7212694961 & 0.4564889697 & -0.1260828618 \\ \text { C4A } & 6.0 & -1.6259391788 & -1.2164290814 & -0.6791342724 \\ \text { C4A } & 6.0 & 1.6259391788 & 1.2164290814 & -0.6791342724 \\ \text { C5 } & 6.0 & -1.6216119952 & -1.6196883110 & -2.0068941492 \\ \text { C5 } & 6.0 & 1.6216119952 & 1.6196883110 & -2.0068941492 \\ \text { C6 } & 6.0 & -0.5778137012 & -2.4370797135 & -2.4869248038 \\ \text { C6 } & 6.0 & 0.5778137012 & 2.4370797135 & -2.4869248038 \\ \text { C7 } & 6.0 & 0.4320838020 & -2.8407854978 & -1.6453324021 \\ \text { C7 } & 6.0 & -0.4320838020 & 2.8407854978 & -1.6453324021 \\ \text { C8 } & 6.0 & 0.4418913125 & -2.4329209036 & -0.3027389678 \\ \text { C8 } & 6.0 & -0.4418913125 & 2.4329209036 & -0.3027389678 \\ \text { C8A } & 6.0 & -0.5693204749 & -1.6215413558 & 0.1894736242 \\ \text { C8A } & 6.0 & 0.5693204749 & 1.6215413558 & 0.1894736242 \\ \text { O } & 8.0 & -1.7891559556 & -0.3215056534 & 3.3402432429 \\ \text { O } & 8.0 & 1.7891559556 & 0.3215056534 & 3.3402432429 \\ \text { H3 } & 1.0 & -3.6715356665 & 0.3962830549 & 1.5844305938 \\ \text { H3 } & 1.0 & 3.6715356665 & -0.3962830549 & 1.5844305938 \\ \text { H4 } & 1.0 & -3.5248336193 & -0.1635974188 & -0.7900285767 \\ \text { H4 } & 1.0 & 3.5248336193 & 0.1635974188 & -0.7900285767 \\ \text { H5 } & 1.0 & -2.4274410031 & -1.3330678586 & -2.6682565640 \\ \text { H5 } & 1.0 & 2.4274410031 & 1.3330678586 & -2.6682565640 \\ \text { H6 } & 1.0 & -0.5885470936 & -2.7624576299 & -3.5175672300 \\ \text { H6 } & 1.0 & 0.5885470936 & 2.7624576299 & -3.5175672300 \\ \text { H7 } & 1.0 & 1.2153293167 & -3.4897447320 & -2.0105128593 \\ \text { H7 } & 1.0 & -1.2153293167 & 3.4897447320 & -2.0105128593 \\ \text { H8 } & 1.0 & 1.2277453654 & -2.7797626966 & 0.3541237824 \\ \text { H8 } & 1.0 & -1.2277453654 & 2.7797626966 & 0.3541237824 \\ \text { H1 } & 1.0 & 0.0740424774 & -1.6058120681 & 2.2878823060 \\ \text { H1 } & 1.0 & -0.0740424774 & 1.6058120681 & 2.2878823060 \\ \text { HOH } & 1.0 & -2.5960219780 & 0.0706460072 & 3.6631248994 \\ \text { HOH } & 1.0 & 2.5960219780 & -0.0706460072 & 3.6631248994\end{array}$




\section{Result of MP2/cc-pVDz calculation}

Structure 3b(E=-918.868943 a.u., 0 imaginary frequences)

$\begin{array}{lrrrr}\mathrm{C} & 6.0 & 0.0212992604 & 0.7759662638 & 1.2747960588 \\ \mathrm{C} & 6.0 & -0.0212992604 & -0.7759662638 & 1.2747960588 \\ \mathrm{C} & 6.0 & 1.3280254444 & 1.3420369951 & 1.7492182000 \\ \mathrm{C} & 6.0 & -1.3280254444 & -1.3420369951 & 1.7492182000 \\ \mathrm{C} & 6.0 & 1.7950499282 & 2.5837315943 & 1.2945115309 \\ \mathrm{C} & 6.0 & -1.7950499282 & -2.5837315943 & 1.2945115309 \\ \mathrm{C} & 6.0 & 1.1160739147 & 3.2242624511 & 0.2568853430 \\ \mathrm{C} & 6.0 & -1.1160739147 & -3.2242624511 & 0.2568853430 \\ \mathrm{C} & 6.0 & -0.0304990896 & 2.6791560812 & -0.3972201162 \\ \mathrm{C} & 6.0 & 0.0304990896 & -2.6791560812 & -0.3972201162 \\ \mathrm{C} & 6.0 & -0.6070509862 & 3.3565895297 & -1.5080237839 \\ \mathrm{C} & 6.0 & 0.6070509862 & -3.3565895297 & -1.5080237839 \\ \mathrm{C} & 6.0 & -1.6838558450 & 2.7808363143 & -2.1842167411 \\ \mathrm{C} & 6.0 & 1.6838558450 & -2.7808363143 & -2.1842167411 \\ \mathrm{C} & 6.0 & -2.1793995805 & 1.5242164284 & -1.7712888578 \\ \mathrm{C} & 6.0 & 2.1793995805 & -1.5242164284 & -1.7712888578 \\ \mathrm{C} & 6.0 & -1.6163172447 & 0.8501003837 & -0.6723557304 \\ \mathrm{C} & 6.0 & 1.6163172447 & -0.8501003837 & -0.6723557304 \\ \mathrm{C} & 6.0 & -0.5366447846 & 1.4111390189 & 0.0246929458 \\ \mathrm{C} & 6.0 & 0.5366447846 & -1.4111390189 & 0.0246929458 \\ \mathrm{O} & 8.0 & 1.9116811050 & 0.6283134613 & 2.6867127488 \\ \mathrm{O} & 8.0 & -1.9116811050 & -0.6283134613 & 2.6867127488 \\ \mathrm{H} & 1.0 & 2.6967002901 & 3.0302324217 & 1.7280540745 \\ \mathrm{H} & 1.0 & -2.6967002901 & -3.0302324217 & 1.7280540745 \\ \mathrm{H} & 1.0 & 1.4964769899 & 4.1948216313 & -0.0887748273 \\ \mathrm{H} & 1.0 & -1.4964769899 & -4.1948216313 & -0.0887748273 \\ \mathrm{H} & 1.0 & -0.2020902194 & 4.3263760848 & -1.8174654167 \\ \mathrm{H} & 1.0 & 0.2020902194 & -4.3263760848 & -1.8174654167 \\ \mathrm{H} & 1.0 & -2.1379018605 & 3.2941992020 & -3.0371072319 \\ \mathrm{H} & 1.0 & 2.1379018605 & -3.2941992020 & -3.0371072319 \\ \mathrm{H} & 1.0 & -3.0161427900 & 1.0685065705 & -2.3110745673 \\ \mathrm{H} & 1.0 & 3.0161427900 & -1.0685065705 & -2.3110745673 \\ \mathrm{H} & 1.0 & -2.0233286223 & -0.1256698175 & -0.3840813174 \\ \mathrm{H} & 1.0 & 2.0233286223 & 0.1256698175 & -0.3840813174 \\ \mathrm{H} & 1.0 & 2.6982785768 & 1.0835981140 & 3.0505852948 \\ \mathrm{H} & 1.0 & -2.6982785768 & -1.0835981140 & 3.0505852948 \\ \mathrm{H} & 1.0 & -0.6463605208 & 1.1135496451 & 2.1060523931 \\ \mathrm{H} & 1.0 & 0.6463605208 & -1.1135496451 & 2.1060523931\end{array}$




\section{Results of DFT/PBE/ $\Lambda 1$ calculations of $\mathbf{3 c}, \mathbf{8}$ and $\mathbf{9}$}

DFT energy and ZPE are in a.u., free energy G correction is in $\mathrm{kcal} / \mathrm{mol}$, dipole moment is in Debyes

Dication 3c

38

Energy -920.7219608892 Charge 2 Dipole 3.874972 ZPE 0.299324 G(298) 159.35

C $\quad 0.78669165-0.72202622-0.54507218$

C 1.97337569

C $\quad 1.97337569 \quad 1.65008877-0.49728171$

C $\quad 1.83319591 \quad 3.03063885 \quad-0.53177107$

C $\quad 0.56122720 \quad 3.59077158-0.48463756$

C $\quad-0.63052452 \quad 2.82887956-0.39332901$

C $-1.88697894 \quad 3.48145306-0.31351332$

C $\quad-3.05338743 \quad 2.73976141 \quad-0.21440823$

C $-2.97740139-1.34073371-0.19726071$

C $\begin{array}{lll}-1.74337475 & 0.67782242 & -0.28104684\end{array}$

C $-0.56008800 \quad 1.40174661-0.37484165$

$\begin{array}{lll}3.13733403 & 1.02665301 & -0.50634748\end{array}$

$\begin{array}{lll}1.00120596 & -0.58495083 & 0.30450290\end{array}$

$\begin{array}{lll}0.71338495 & -0.36328159 & 1.76186239\end{array}$

$\begin{array}{lll}0.11665603 & -1.33212202 & 2.55536899\end{array}$

$\begin{array}{lll}-0.32595697 & -2.51649748 & 1.97287249\end{array}$

$\begin{array}{lll}-0.20654656 & -2.80684056 & 0.59160088\end{array}$

$\begin{array}{llll}-0.68979460 & -4.03854088 & 0.07843970\end{array}$

$\begin{array}{llll}-0.55352122 & -4.33475751 & -1.26773973\end{array}$

$\begin{array}{lll}0.07546668 & -3.41065949 & -2.11387255\end{array}$

$0.55898231-2.18857926-1.62374932$

$0.41593664-1.85916020-0.28029912$

$\begin{array}{lll}1.13632595 & 0.80539874 & 2.21172326\end{array}$

$\begin{array}{lll}2.71557590 & 3.67604507 & -0.57970471\end{array}$

$\begin{array}{lll}0.47549875 & 4.68297722 & -0.50578127\end{array}$

$\begin{array}{llll}-1.92253326 & 4.57420106 & -0.33551137\end{array}$

$\begin{array}{llll}-4.02327984 & 3.23840001 & -0.15637459\end{array}$

$\begin{array}{lll}-3.89523044 & 0.75137152 & -0.12502216\end{array}$

$\begin{array}{llll}-1.73166015 & -0.41477670 & -0.28689839\end{array}$

$\begin{array}{llll}\mathrm{H} & 3.90179622 & 1.64571311 & -0.55941726\end{array}$

$\begin{array}{llll}-0.01688200 & -1.16610919 & 3.62880555\end{array}$

$\begin{array}{llll}-0.79801679 & -3.26552071 & 2.61847423\end{array}$

$\begin{array}{llll}-1.15961664 & -4.75235201 & 0.76080916\end{array}$

$\begin{array}{llll}-1.92080122 & -5.28267955 & -1.66680588\end{array}$

$\begin{array}{lll}0.19894259 & -3.64475541 & -3.17453386\end{array}$

$\begin{array}{lll}1.06057600 & -1.51455440 & -2.32445342\end{array}$

$\begin{array}{lll}0.99925418 & 0.90427728 & 3.18179721\end{array}$

$\begin{array}{lll}0.80933388 & 0.39031043 & -1.60784574\end{array}$

H $\quad 2.10217426-0.76136766 \quad 0.29663843$ 


\section{Dication 8}

38

Energy -920.7446048529 Charge 2 Dipole 5.597855 ZPE 0.298860 G (298) 158.20

Energy -920.7446048529 Charge 2 Dipole
C $1.44718227 \quad 0.76426260 \quad 2.44045286$

$0.54258146 \quad 2.14659143$

$-0.34277394$

0.35942585

0.82347062

0.59021371

0.40247724

$-0.20796806$

1.95775073

0.62994838

0.07367151

2.38979982

0.81272951

1.41538353

3. 84308444

1.05112169

1.70395725

2.92429414

0.67818648

0.89450696

$-0.97842837$

4.75253427

1.07965606

1.39974665

1.40450314

0.22358035

0.52871229

0.07065370

$-3.45734806$

$-3.74391332$

$-3.11740608$

$-2.13809134$

$-1.01718833$

.95068159

$-0.31202674$

0.52882771

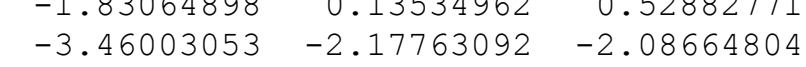

$-2.84783715$

$-3.38806079$

$-1.80386251$

$-1.88876361$

$-1.53576309$

$-2.17321259$

$-3.45347713$

$-0.78277791$

$-2.31550351-0.04351930$

$2.46814185 \quad 0.76347197$

$1.28974364 \quad 0.83154698$

$\begin{array}{lll}6.00965184 & 1.28974364 & 0.83154698 \\ 1.75350643 & 0.89785549 & 3.48168459\end{array}$

$\begin{array}{lll}-0.61868888 & 0.51072655 & 2.97055043\end{array}$

$\begin{array}{lll}0.28186242 & 0.25430330 & -1.24744861\end{array}$

$\begin{array}{lll}3.97793886 & 2.00597923 & 2.25323585\end{array}$

$\begin{array}{lll}4.23080063 & 0.28619991 & 2.40826045\end{array}$

$\begin{array}{lll}2.57401915 & 0.53515090 & -2.00666248\end{array}$

$\begin{array}{lll}4.96245443 & 0.91978908 & -1.62714453\end{array}$

$\mathrm{H} \quad-3.97160985 \quad 2.33083210 \quad-1.22501937$

$\mathrm{H}-4.49403600 \quad 0.25365008 \quad-2.42544763$

$\mathrm{H} \quad-2.32492504 \quad-0.05544957 \quad 1.50943017$

$\mathrm{H} \quad-4.21591161-2.10568561 \quad-2.87355187$

$\mathrm{H}-3.11313200 \quad-4.28612699 \quad-2.36567882$

$\begin{array}{llll}-1.41010831 & -4.40891635 & -0.55256888\end{array}$

$\mathrm{H} \quad-0.78897861 \quad-2.40271335 \quad 0.74983505$

$\mathrm{H} \quad-2.68566383 \quad 3.26469913 \quad 0.49488575$

$\mathrm{H} \quad 6.60285272 \quad 1.30690540 \quad 0.04480578$ 


\section{Dication 9}

38

C $3.21611780=-3.61332435$

$2.35497345 \quad-2.54440116 \quad 3.77791439$

$1.54807567 \quad-1.74624216 \quad 2.79678555$

$\begin{array}{lll}1.64759474 & -2.02704898 & 1.40698916\end{array}$

$2.50756266-3.08111505 \quad 0.97503055$

$3.27147195-3.85639319$

$\begin{array}{llll}0.69301186 & -0.72952761 & 3.21872182\end{array}$

$\begin{array}{lll}0.05124568 & -0.00584666 & 2.28778356\end{array}$

$\begin{array}{llll}\text { C } & 0.03504420 & -0.26614061 & 0.90136488\end{array}$

$\begin{array}{rrrr}\text { C } & 0.89070525 & -1.28001960 & 0.47398997\end{array}$

C $\quad-0.77951099 \quad 0.52282963-0.06018516$

$\begin{array}{llll}\text { C } & -2.14710769 & 0.76733871 & 0.19911748\end{array}$

$\begin{array}{llll}\text { C } & -2.93969958 & 1.50841191 & -0.67660746\end{array}$

C $-2.39094755 \quad 2.03828251 \quad-1.84321800$

C $-1.01658019 \quad 1.80684872 \quad-2.12219944$

$\begin{array}{llll}\text { C } & -0.22671511 & 1.04766077 & -1.22712333\end{array}$

C $-3.21960154 \quad 2.84528420 \quad-2.79861850$

C $-2.51726427 \quad 3.37287207 \quad-3.99740903$

C $\quad-1.15852028 \quad 3.12362546 \quad-4.22203916$

C $-0.44847231 \quad 2.36541742-3.30645422$

$\begin{array}{llll}0 & -3.28196601 & 4.07603063 & -4.79893142\end{array}$

$\begin{array}{llll}0 & 3.89835964 & -4.28535997 & 4.10518099\end{array}$

$\mathrm{H} \quad-3.99600675 \quad 1.66596702 \quad-0.44167587$

$\mathrm{H} \quad-2.60684386 \quad 0.34789949 \quad 1.09828278$

$\begin{array}{llll}H & 0.83486322 & 0.90507980 & -1.44932746\end{array}$

$\mathrm{H} \quad-4.08797429 \quad 2.25312483-3.15468438$

$\mathrm{H} \quad-3.68431249 \quad 3.70729814 \quad-2.27711787$

$2.18458263-3.50192277$

$\begin{array}{llll}-0.66001843 & 3.52317809 & -5.11010446\end{array}$

$\mathrm{H} \quad-2.80137224 \quad 4.41889639 \quad-5.58729943$

H $\quad 0.60274382-0.48897713 \quad 4.28163348$

$\begin{array}{llll}\mathrm{H} & -0.70075624 & 0.79852351 & 2.64402731\end{array}$

H $\quad 0.96361415-1.53156679-0.58811257$

H $\quad 3.00598783 \quad-1.87641093 \quad 4.37913631$

$\mathrm{H} \quad 1.69247282-3.01600686 \quad 4.53271570$

$\mathrm{H} \quad 2.56555869-3.29029690 \quad-0.09882900$

H $\quad 3.90999495 \quad-4.65026831 \quad 1.43245350$

$\mathrm{H} \quad 4.46250855-4.99020569 \quad 3.71151175$ 
Thermogravimetric analysis (TGA) and differential scanning calorimetry (DSC) measurements for $\mathbf{1}$ and $\mathbf{5}$

Thermogravimetric analysis (TGA) and differential scanning calorimetry (DSC) measurements were performed on a NETZSCH STA 409 instrument. Temperature and heat flow calibration was calibrated according to ISO 11357-1 standard by the temperatures and enthalpies of phase transitions of standard substances from calibration set from NETZSCH (C6H5COOH (99,5\%), RbNO3 99,99\%), In (99,99+\%), Sn (99,99\%), Bi (99,9995\%), Zn (99,999\%). Samples ( 5-6 mg) were put in aluminum crucibles $(\mathrm{V}=25 \mu \mathrm{l}, \mathrm{d}=6 \mathrm{~mm})$ with pierced lid and were heated at a heating rate of $50{ }^{\circ} \mathrm{C} / \mathrm{min}$ under He flow $30 \mathrm{ml} / \mathrm{min}$. The obtained

data were processed using NETZSCH Proteus Thermal Analysis software. Temperature of maximal rate of decomposition ( $\left.T_{\max }\right)$ was determined from the dTG diagrams. The decomposition enthalpies $\Delta_{d} \mathrm{H}$ (DSC)

were determined from DSC curves .

Figure S5 . TG (- $(-)$ dTG (--------), DSC (- curves for $\mathbf{1}$ (left) and $\mathbf{5}$ (right)

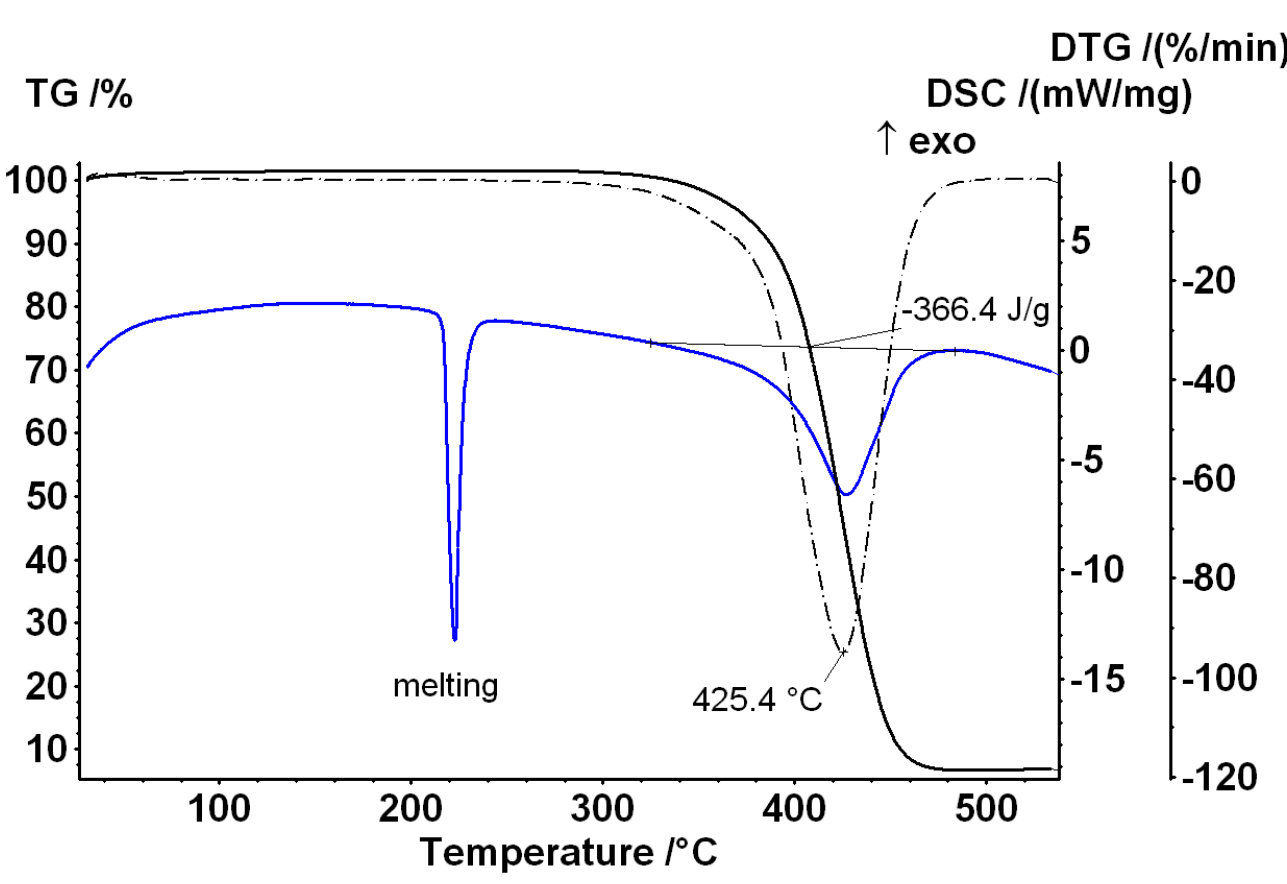

$T H$

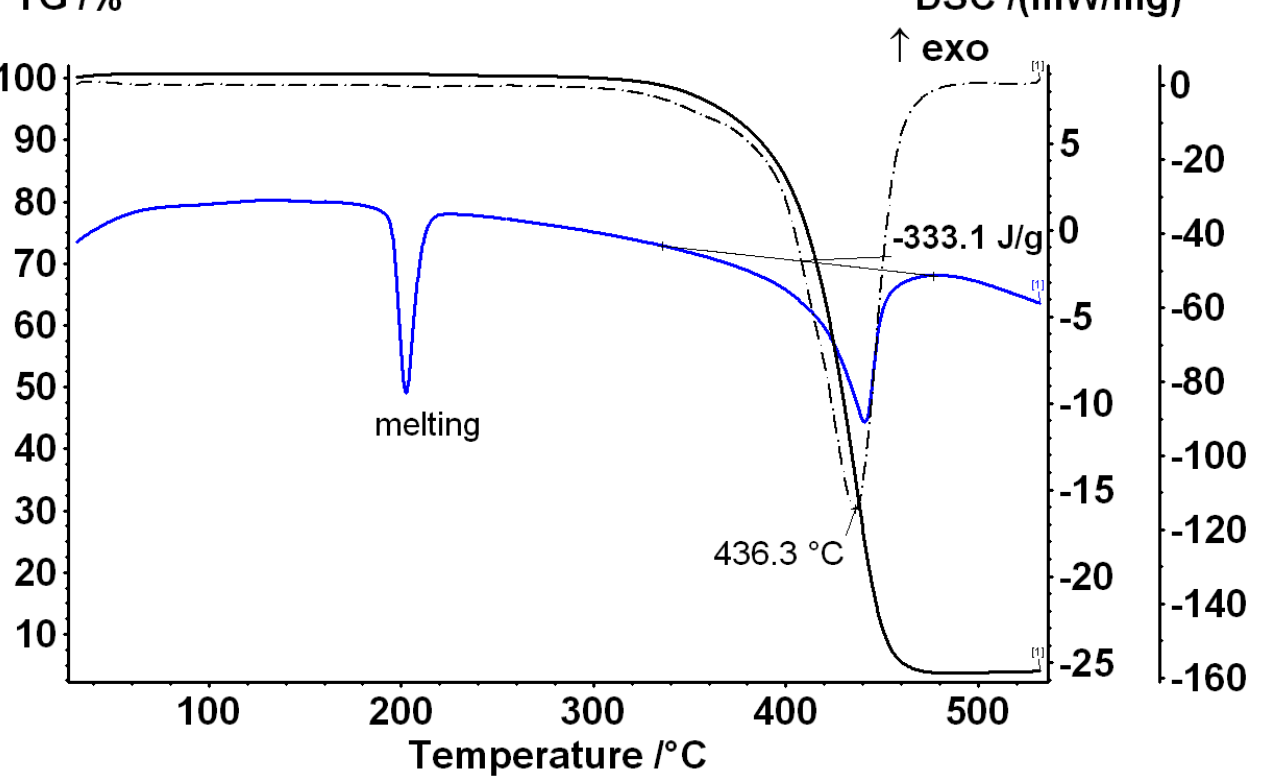


NMR data on the mixtures obtained by thermolysis of $\mathbf{1}$ and $\mathbf{5}$

(under Ar at $400{ }^{\circ} \mathrm{C}$ for $1 \mathrm{~h}$, pages S41-53)

NMR signal assignments for 6, 1 and 2-naphthol (pages S41-S42)

Dinaphthofuran 6 (the corresponding signals are marked by $\mathbf{A}$ on spectra)

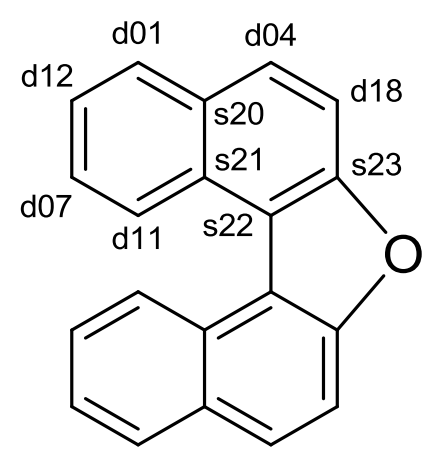

Experiment Bruker_1, 1D 13C d01 129.9

d04 129.0

d07 126.8

d11 125.6

d12 124.9

d18 112.9

s20 131.7

s21 128.7

s22 119.2

s23 154.6

Experiment Bruker_2, 1D 1H d01-H 8.19 (d)

d04-H 8.12 (d)

d07-H $7.82(\mathrm{t})$

d11-H $9.20(\mathrm{~d})$

d12-H $7.64(\mathrm{t})$

d18-H 7.95 (d)
Experiment Bruker_9, 2D 13C-1H via onebond (HSQC)

$\mathrm{d} 01-\mathrm{H}$ - d01

d04-H - d04

d07-H - d07

d11-H - d11

d12-H - d12

d18-H - d18

Experiment Bruker_6, 2D 1H-13C via onebond (H-C correlation)

$\mathrm{d} 01-\mathrm{d} 01-\mathrm{H}$

d04 - d04-H

d07 - d07-H

d11 - d11-H

d12 - d12-H

d18 - d18-H

Experiment Bruker_7, 2D 1H-1H via Jcoupling (COSY)

d01-H - d07-H d12-H

d04-H - d01-H?(weak) d11-H(weak) d18$\mathrm{H}$

d07-H - d01-H d11-H d12-H

d11-H - d04-H(weak) d07-H d12-H

d12-H - d01-H d07-H d11-H

d18-H - d04-H

Experiment Bruker_14, 2D 13C-1H via Jcoupling (HMBC)

d01-H - d04 d07 s20(weak) s21

d04-H - d01 d18(weak) s20(weak) s21 s22(weak) s23

d07-H - d01 d12(weak) s21
d11-H - d01(weak) d12 s20 s21(weak)

$$
\text { s22 }
$$

d12-H - d07 (weak) d11 s20

d18-H - d04(weak) s20 s21(weak) s22

s23(weak)

Experiment Bruker_16,2D 1H-13C via Jmultibond (COLOC)

d01 - d04-H

$\mathrm{d} 04-\mathrm{d} 01-\mathrm{H}$

$\mathrm{d} 07-\mathrm{d} 01-\mathrm{H}$

d12 - d11-H

s20 - d11-H d18-H

s21 - d01-H d04-H

s22 - d11-H d18-H

$\mathrm{s} 23-\mathrm{d} 04-\mathrm{H}$

BINOL 1 (the corresponding signals are marked by $\mathbf{B}$ on spectra)

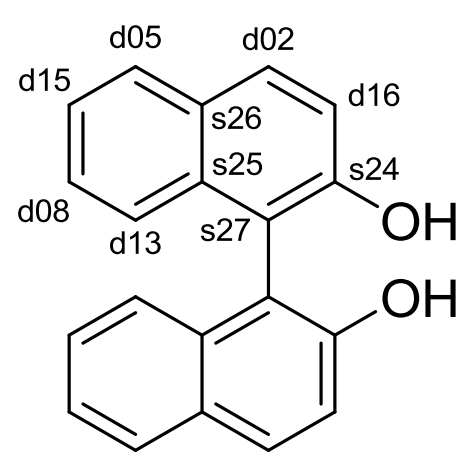

Experiment Bruker_1, 1D 13C d02 129.9

d05 128.2

d08 126.4 d13 124.8

d15 123.0

d16 118.7

s24 153.8

s25 134.8

s26 129.3

s27 114.3

Experiment Bruker_2, 1D 1H

d02-H 7.91 (d)

d05-H 7.87 (d)

$\mathrm{d} 08-\mathrm{H} \quad 7.21(\mathrm{t})$

d13-H $7.05(\mathrm{~d})$

d15-H 7.27 (t)

d16-H 7.35 (d)

Experiment Bruker_9, 2D 13C-1H via onebond (HSQC)

d02-H - d02

d05-H - d05

d08-H - d08

d13-H - d13

d15-H - d15

d16-H - d16

Experiment Bruker_6, 2D 1H-13C via onebond (H-C correlation)

d02 - d02-H

d05 - d05-H

d08 - d08-H

d13 - d13-H

d15 - d15-H

d16 - d16-H 
Experiment Bruker_7, 2D 1H-1H via Jcoupling (COSY)

d02-H - d13-H(weak) d16-H

d05-H - d08-H d15-H

d08-H - d05-H d13-H d15-H

d13-H - d02-H(weak) d08-H d15-H

d15-H - d05-H d08-H d13-H

d16-H - d02-H

Experiment Bruker_8, 2D 1H-1H via through-space (NOESY)

$\mathrm{d} 02-\mathrm{H}-\mathrm{d} 05-\mathrm{H}$

$\mathrm{d} 05-\mathrm{H}-\mathrm{d} 02-\mathrm{H}$

Experiment Bruker_14, 2D 13C-1H via Jcoupling (HMBC)

d02-H - d05 s24 s25 s26(weak)

s27(weak)

d05-H - d02 d08 s25 s26(weak)

d08-H - d05 s25

d13-H - d15 s26 s27

d15-H - d13 s26

d16-H - s24(weak) s26 s27

Experiment Bruker_16, 2D 1H-13C via Jmultibond (COLOC)

d15 - d13-H

$\mathrm{s} 24-\mathrm{d} 02-\mathrm{H}$

s $25-\mathrm{d} 02-\mathrm{H}$

s26 - d16-H

s27 - d13-H d16-H 2-naphthol (the corresponding signals are marked by $\mathbf{C}$ on spectra)

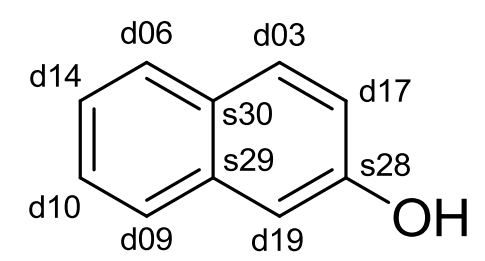

Experiment Bruker_1, 1D 13C

d03 129.6

d06 127.8

d09 126.4

d10 126.3

d14 123.0

d17 118.5

d19 109.1

s28 155.5

s29 135.3

s30 128.8

Experiment Bruker_2, 1D 1H

d03-H $7.76(d)$

d06-H 7.77 (d)

d09-H 7.67 (d)

d10-H $7.38(\mathrm{t})$

d14-H $7.26(\mathrm{t})$

d17-H 7.14 (d)

d19-H $7.20(\mathrm{~s})$

Experiment Bruker_9, 2D 13C-1H via onebond (HSQC)

$\mathrm{d} 03-\mathrm{H}$ - d03

d06-H - d06

d09-H - d09

$\mathrm{d} 10-\mathrm{H}-\mathrm{d} 10$

d14-H - d14

d17-H - d17

d19-H - d19
Experiment Bruker_6, 2D 1H-13C via onebond (H-C correlation)

$\mathrm{d} 03-\mathrm{d} 03-\mathrm{H}$

d06 - d06-H

d09 - d09-H

$\mathrm{d} 10-\mathrm{d} 10-\mathrm{H}$

d14 - d14-H

d17 - d17-H

d19 - d19-H

Experiment Bruker_7, 2D 1H-1H via Jcoupling (COSY)

d03-H - d17-H

d06-H - d10-H(weak) d14-H

d09-H - d10-H d14-H(weak)

d10-H - d06-H(weak) d09-H d14-H

d14-H - d06-H d09-H(weak) d10-H

d17-H - d03-H d19-H

d19-H - d17-H

Experiment Bruker 8, 2D 1H-1H via through-space (NOESY)

d09-H - d19-H

d19-H - d09-H

Experiment Bruker_15, 2D 1H-1H via through-space (NOESY)

d09-H - d19-H

d19-H - d09-H

Experiment Bruker_14, 2D 13C-1H via Jcoupling (HMBC)

d03-H - d06 s28 s29 s30(weak)

d06-H - d03 d10 s29 s30(weak)

d09-H - d14 d19 s30

d10-H - d06 d14(weak) s29

d14-H - d09 s30

d17-H - d19 s28(weak) s30

d19-H - d09 d17 s28(weak) s30
Experiment Bruker_16, 2D 1H-13C via Jmultibond (COLOC)

d09 - d19-H

d17 - d19-H

$\mathrm{s} 28-\mathrm{d} 03-\mathrm{H}$

s29-d03-H 


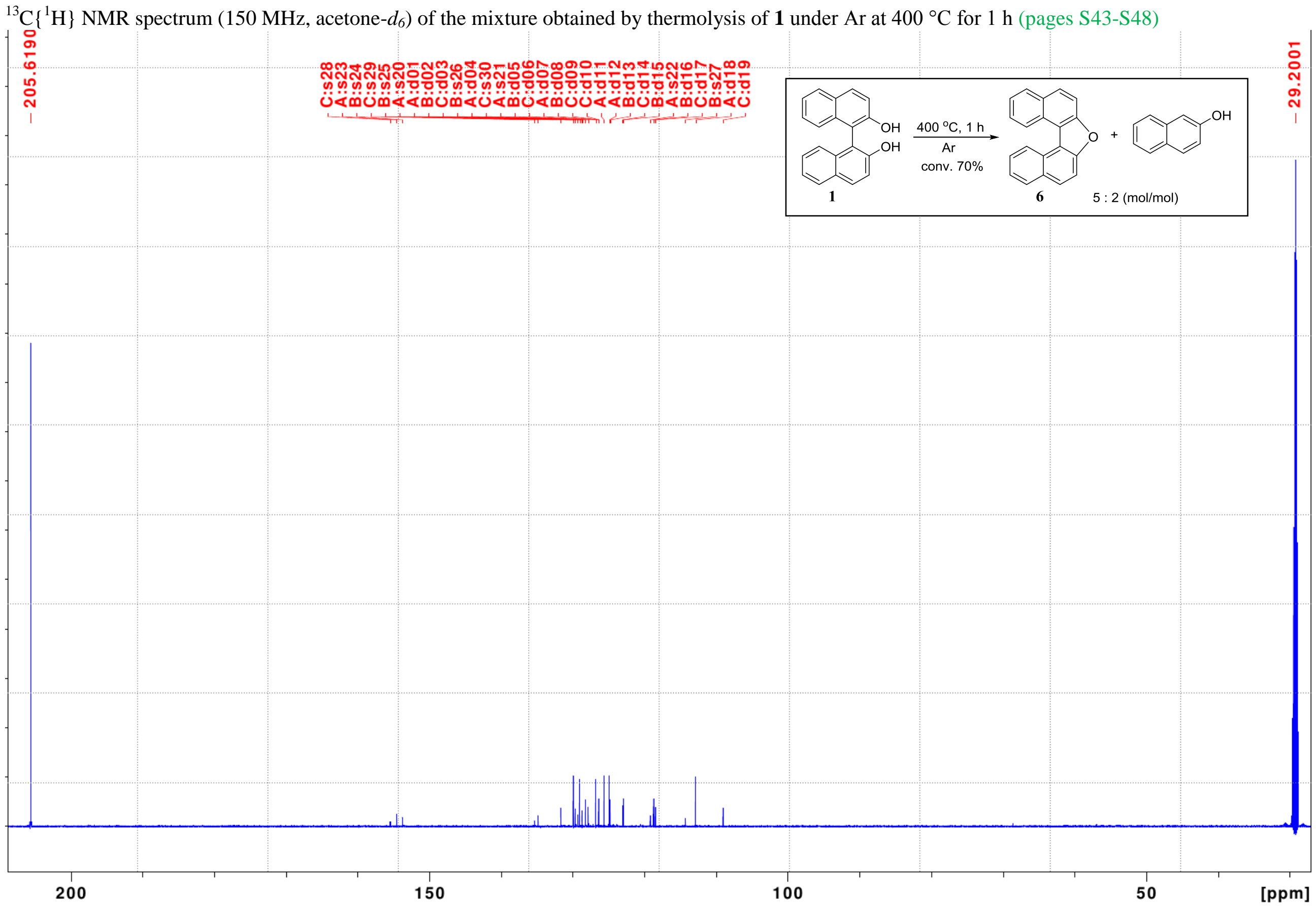




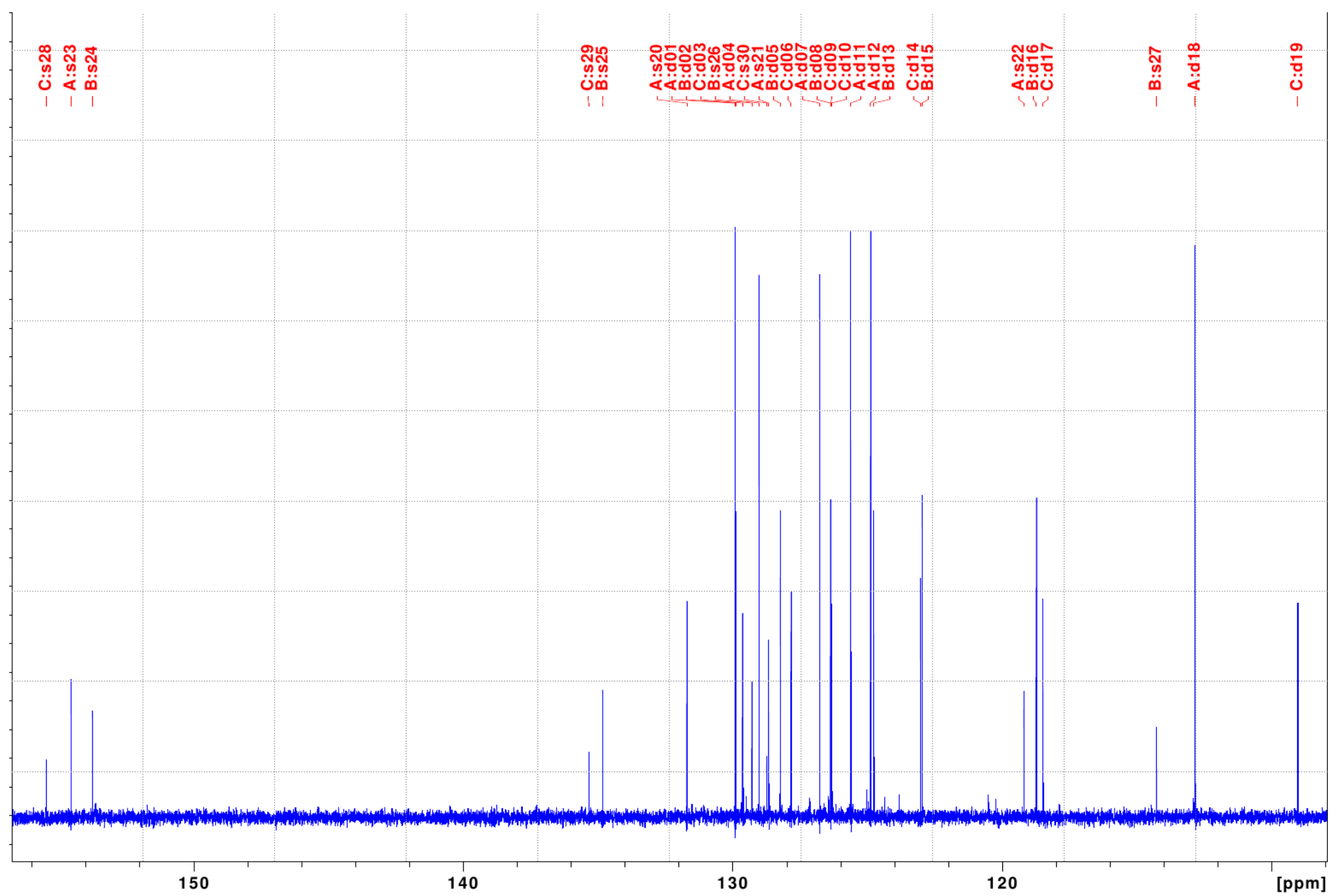




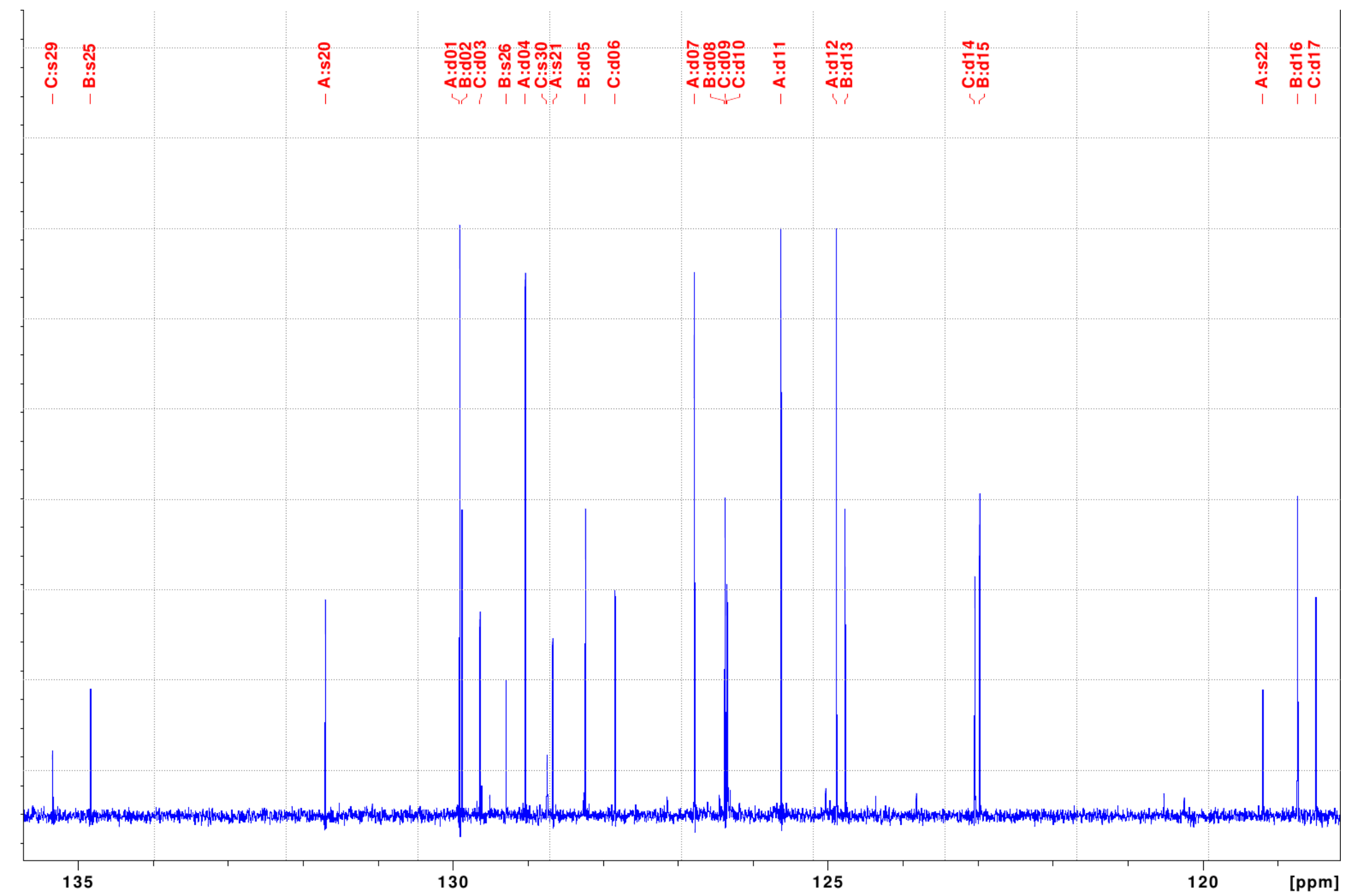




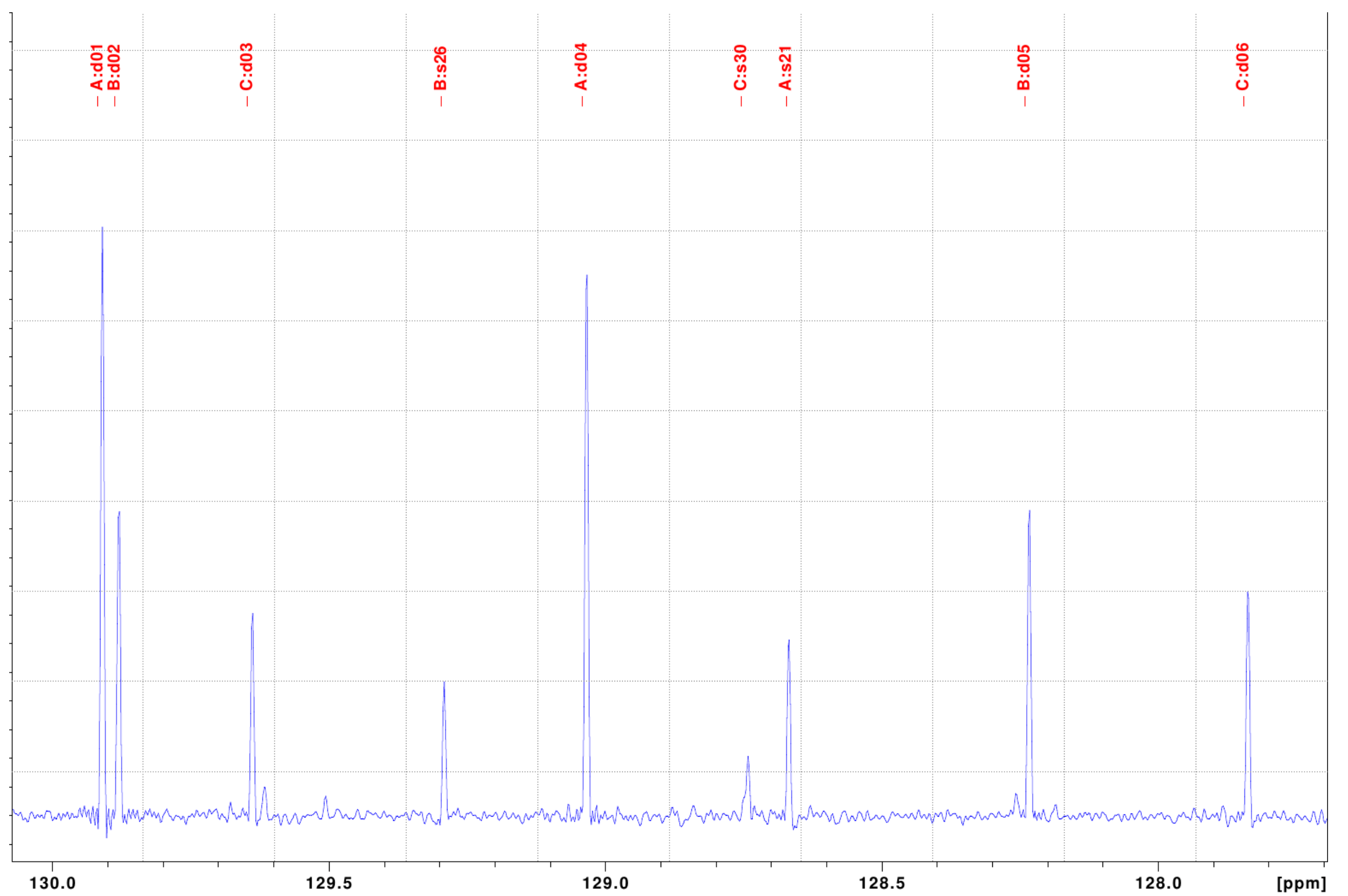




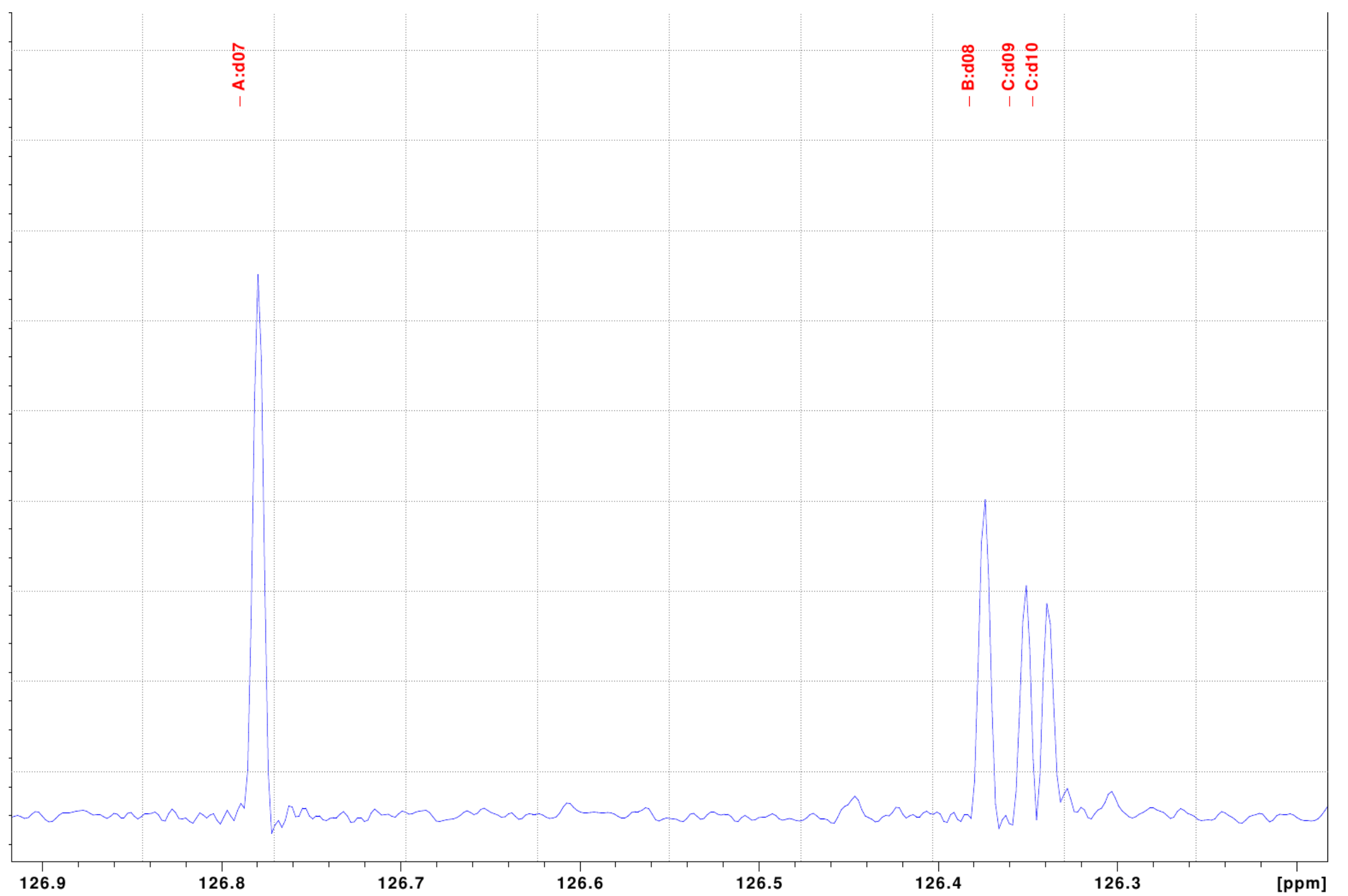




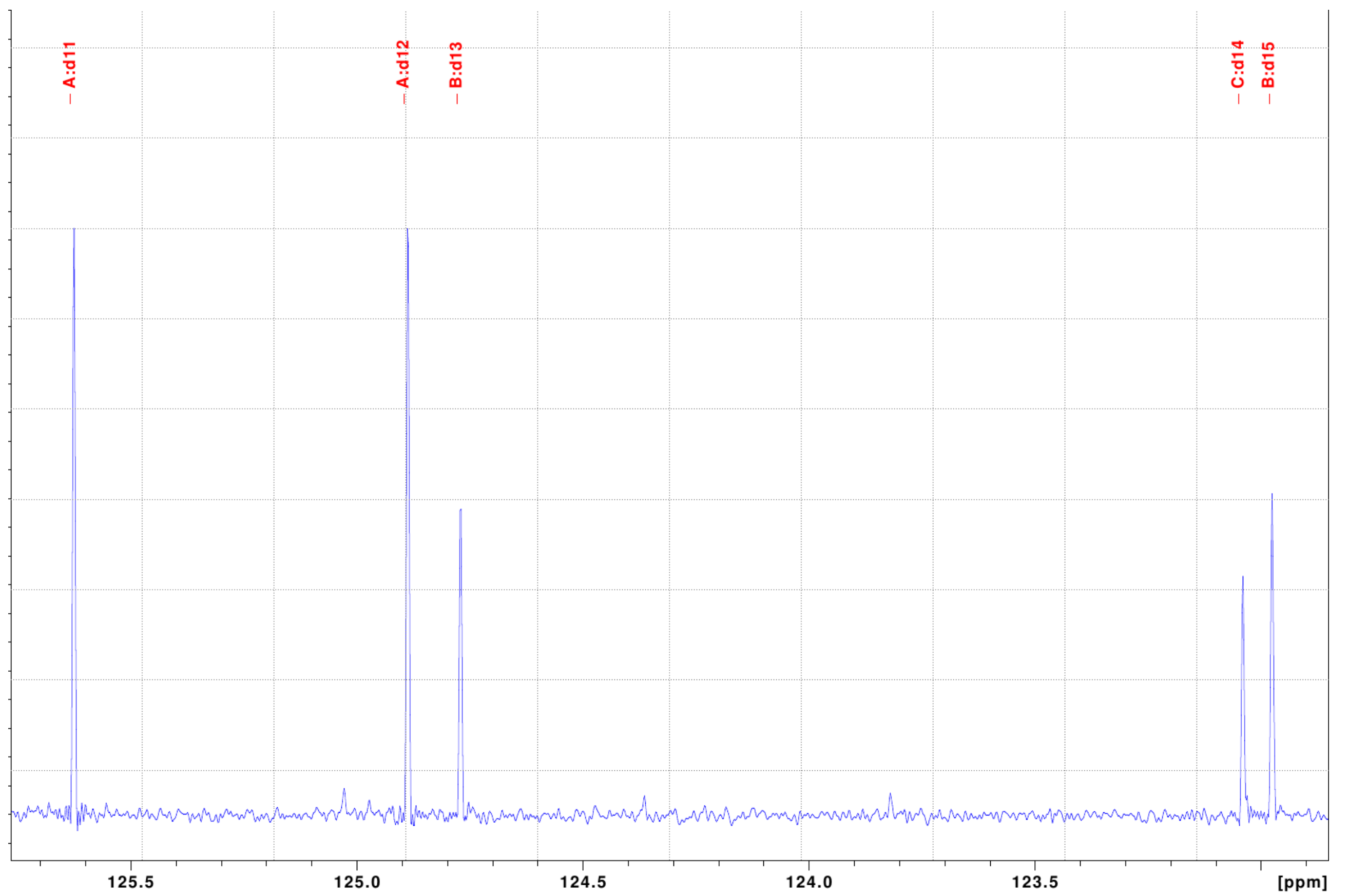


${ }^{1} \mathrm{H}$ NMR spectrum $\left(600 \mathrm{MHz}\right.$, acetone- $\left.d_{6}\right)$ of the mixture obtained by thermolysis of $\mathbf{1}$ under $\mathrm{Ar}$ at $400{ }^{\circ} \mathrm{C}$ for $1 \mathrm{~h}$ (pages S49-S52)

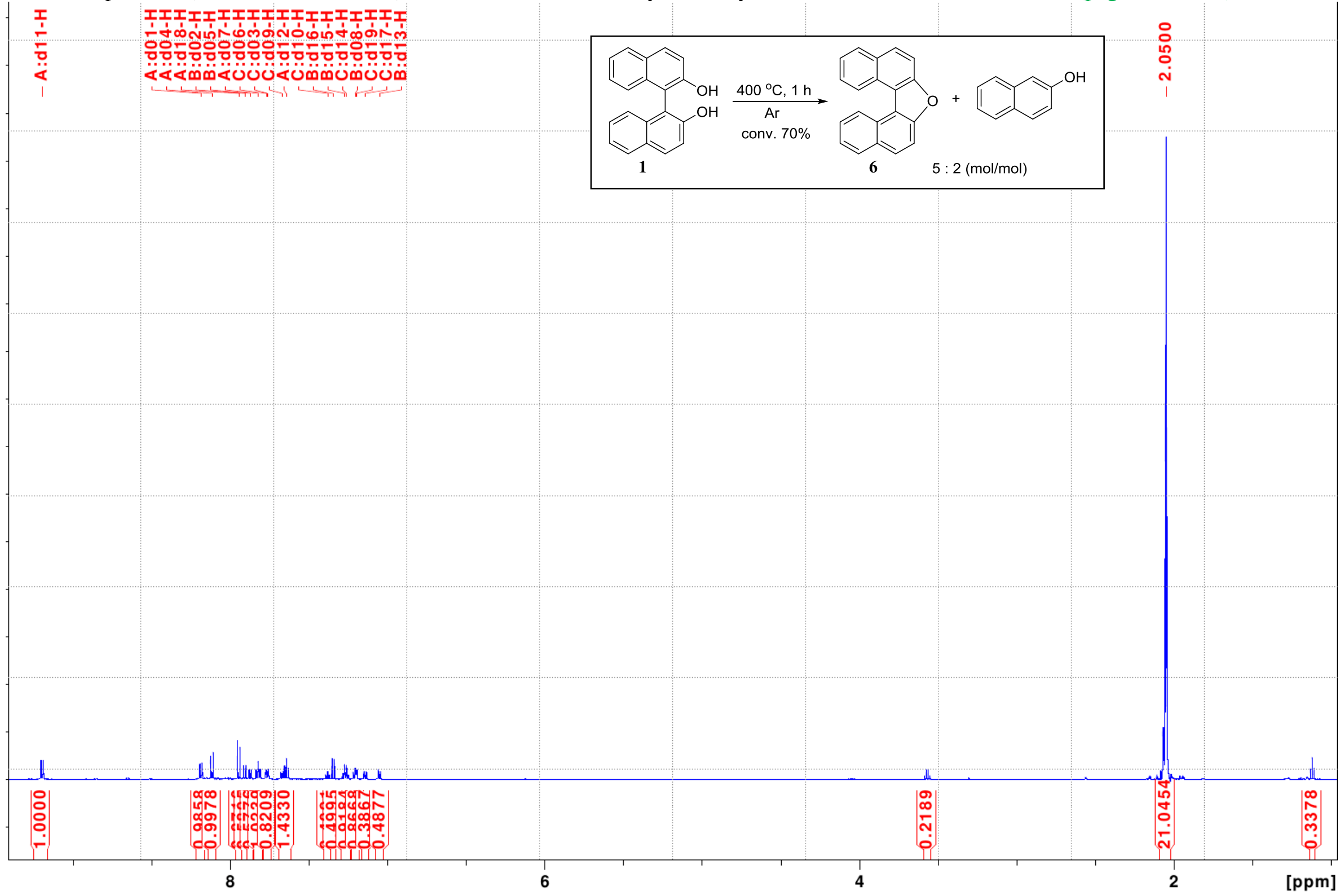




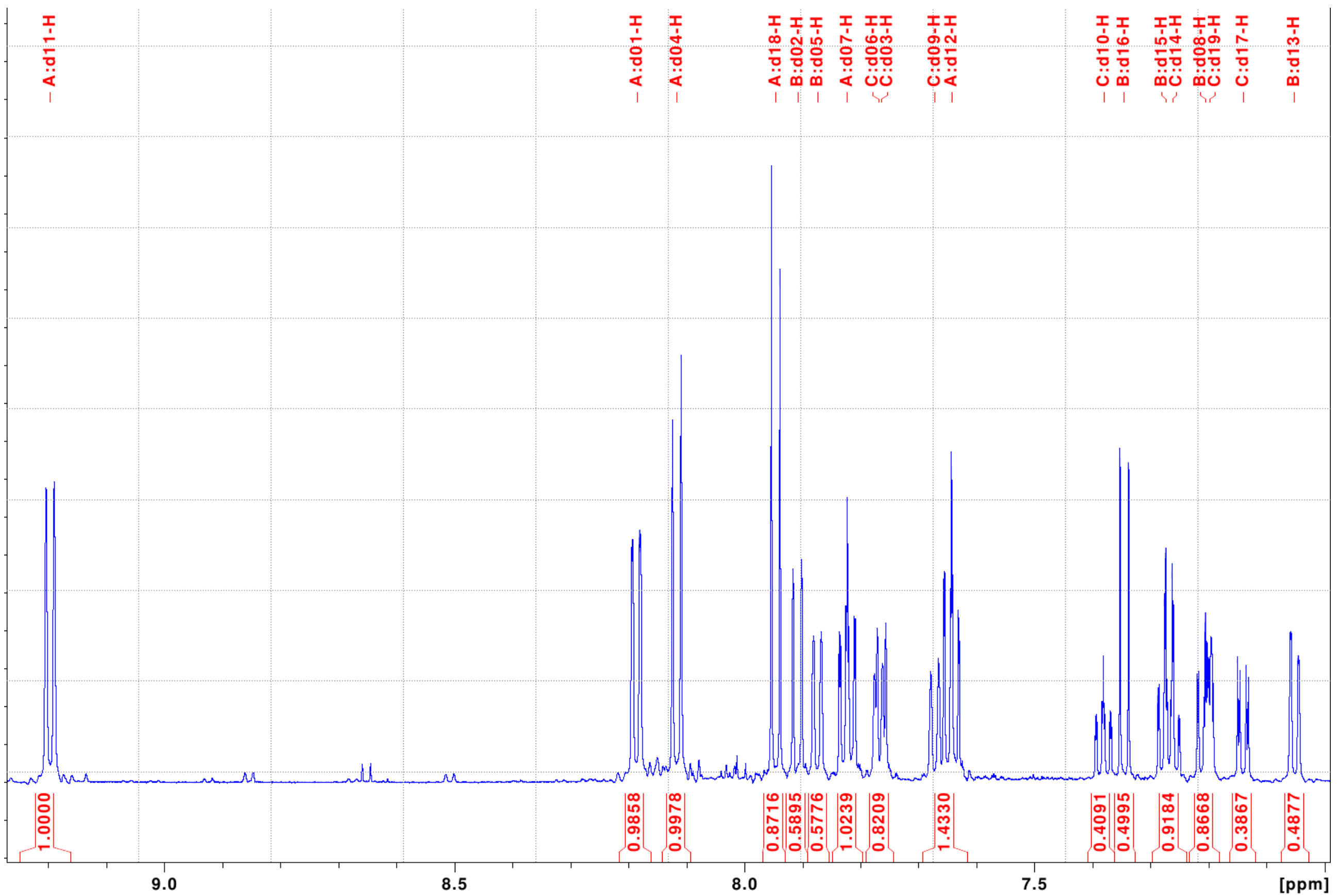




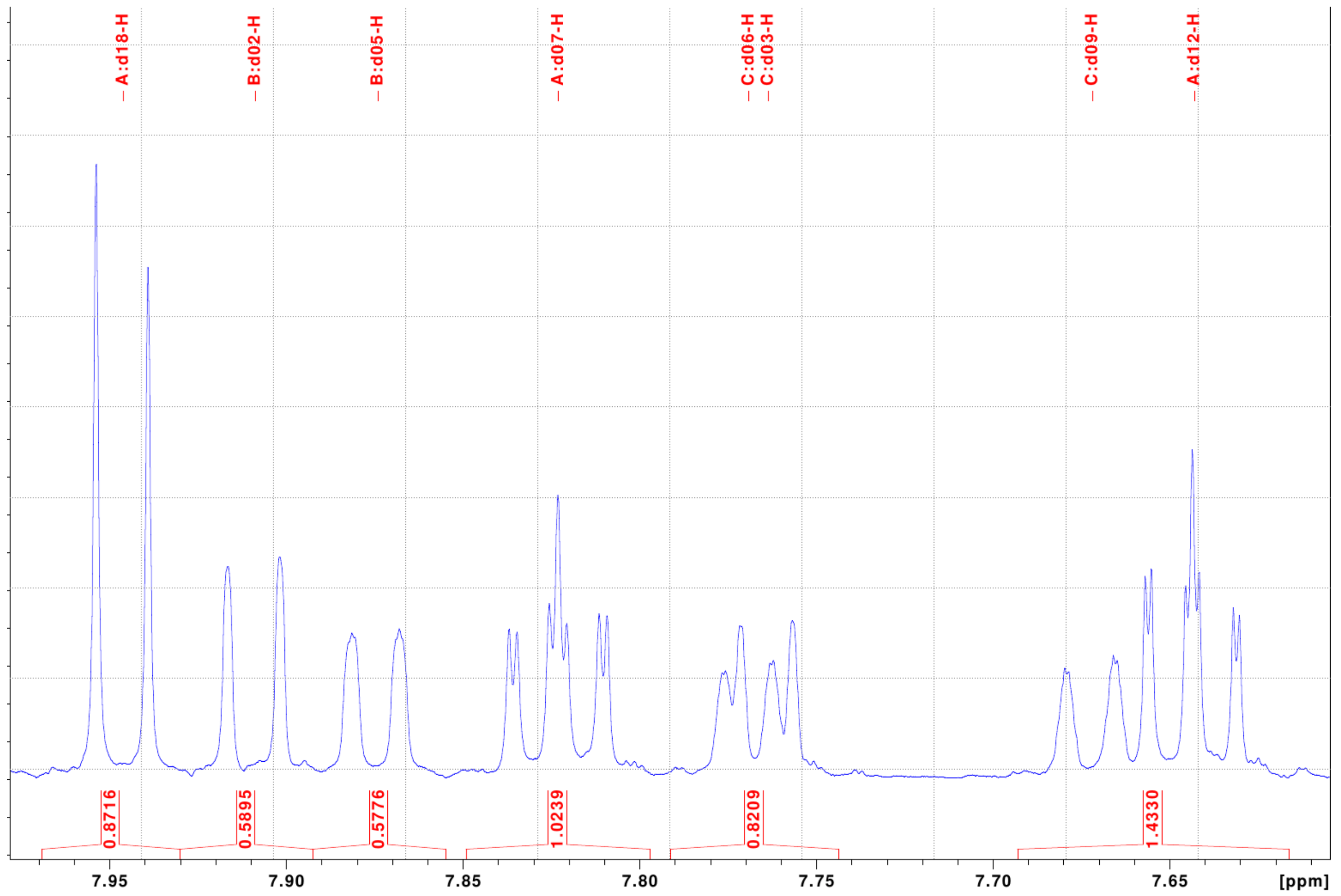




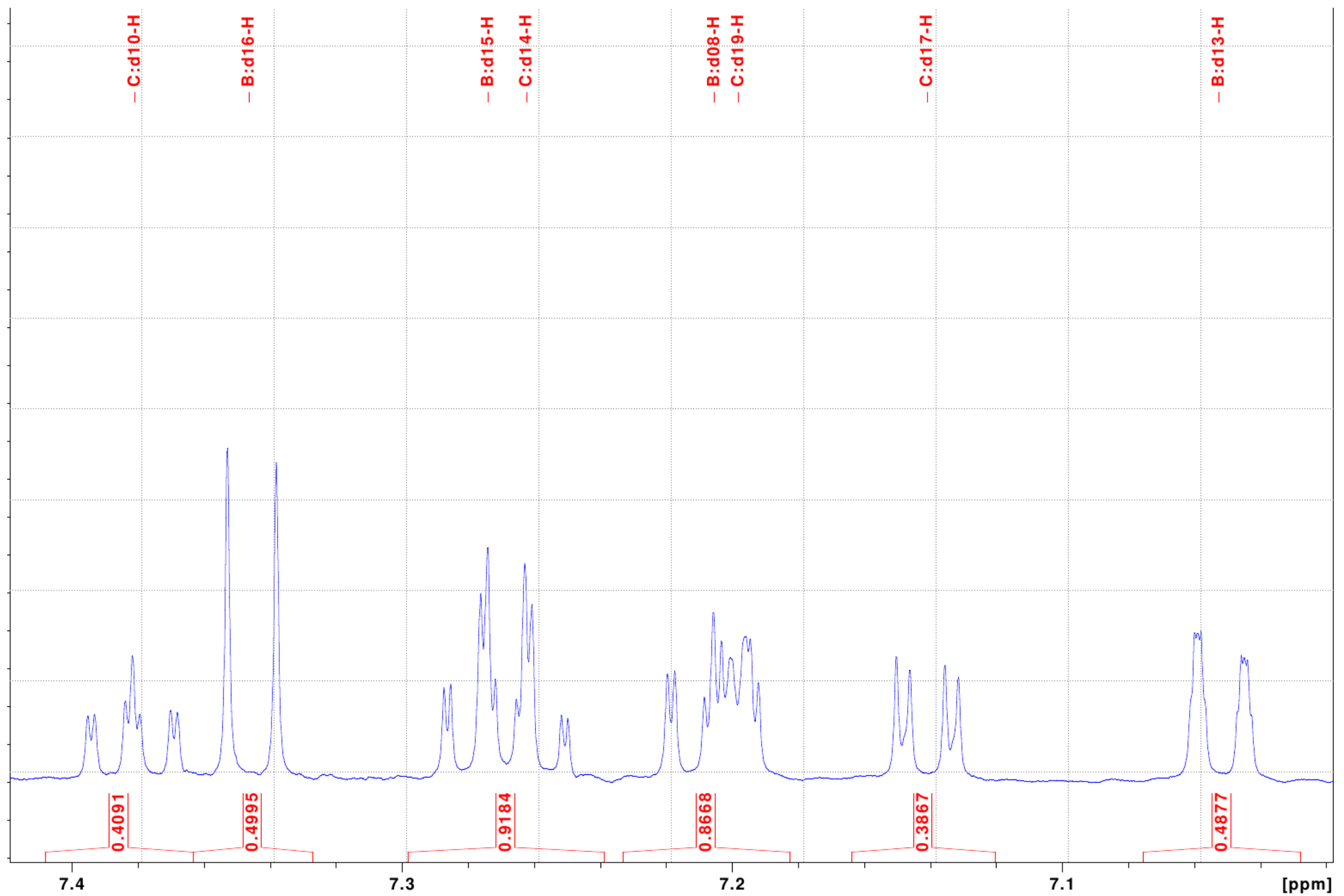


${ }^{1} \mathrm{H}$ NMR spectrum $\left(400 \mathrm{MHz}, \mathrm{CDCl}_{3}\right.$ ) of the reaction mixture obtained by thermolysis of 5 at $400{ }^{\circ} \mathrm{C}$ for $1 \mathrm{~h}$
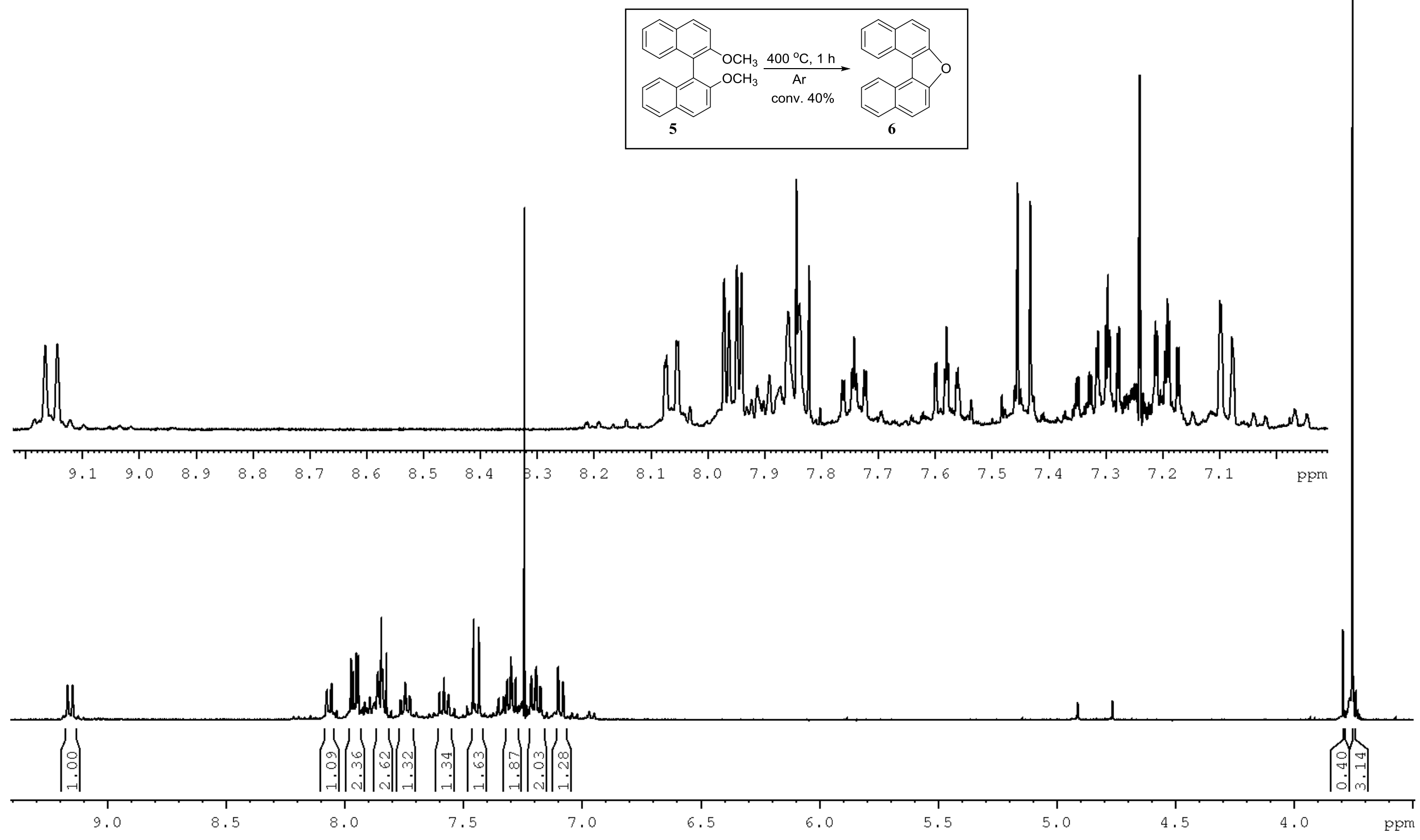
GC-MS data on cleavage of $\mathbf{1}$ and $\mathbf{5}$ in TfOH-CHCl 3

GC-MS data on cleavage of $\mathbf{1}$ in $\mathrm{TfOH}-\mathrm{CHCl}_{3}$ at $0{ }^{\circ} \mathrm{C}$ (pages $54-55$ )
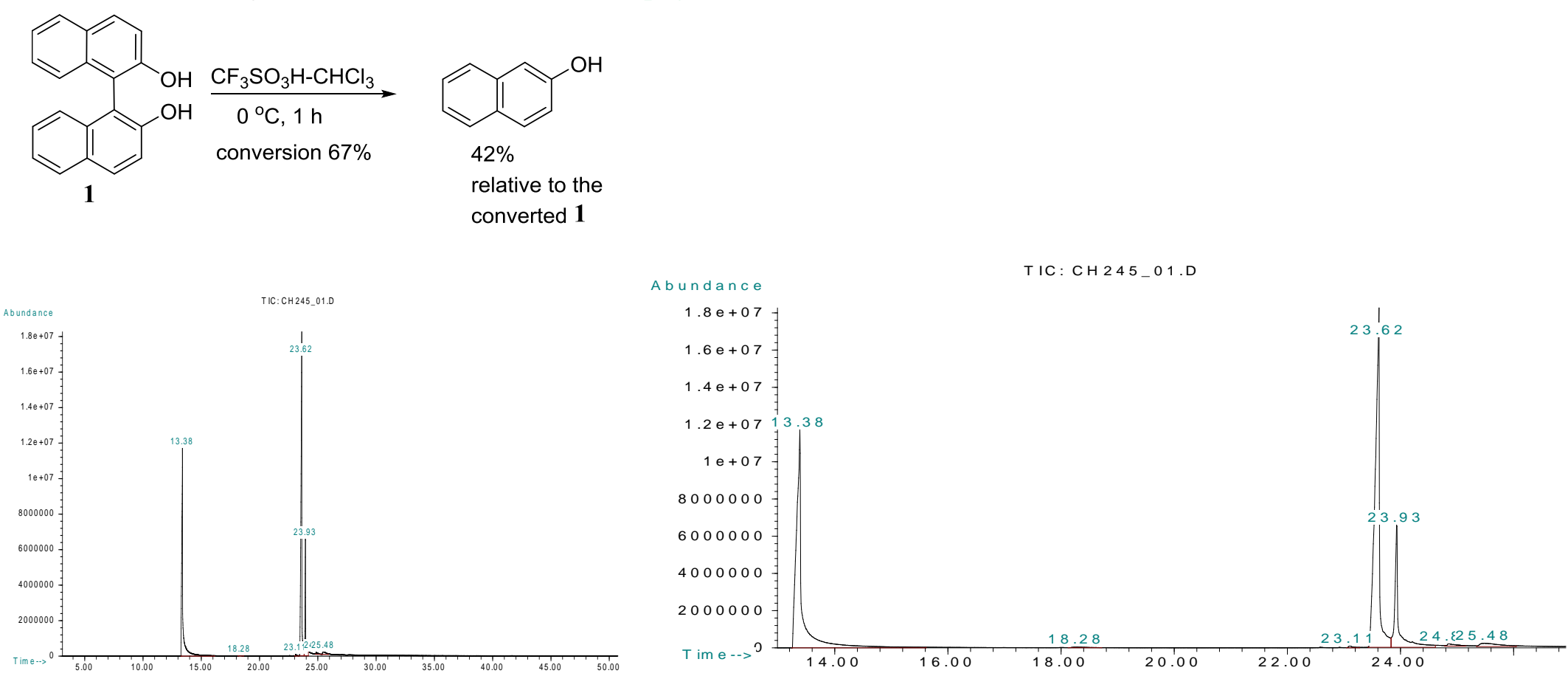

AcqMethod AN10 280

Misc Info: HP-5MS inj $280{ }^{\circ} \mathrm{C} 2 \mathrm{~m} 5010 \mathrm{deg} / \mathrm{min} 35 \mathrm{~min} 280^{\circ} \mathrm{C}$

Retention

Time

Area

Area \% Name

Total Ion Chromatogram

13.382

18.277

83467282

502607

587029

39.464

23.107

23.620

97378754

0.238

0.278

23.930

24.847

25537637

1070363

46.041

2958589

12.074

0.506

25.482

1.399

$\mathrm{MM}=144$ 2-Naphthol

$\mathrm{MM}=214$ - ?

$\mathrm{MM}=416-$ ??

$\mathrm{MM}=286$ 1,1`-Bi-2-naphthol

$\mathrm{MM}=342$ - ??

$\mathrm{MM}=268$ Bis-naphthylfuran

$\mathrm{MM}=286$ - ?? 
Mass spectrum for RT $\mathbf{2 3 . 1}$ min:

Scan $2773(23.100 \mathrm{~min})$ : CH 245_-01.0 (-.)

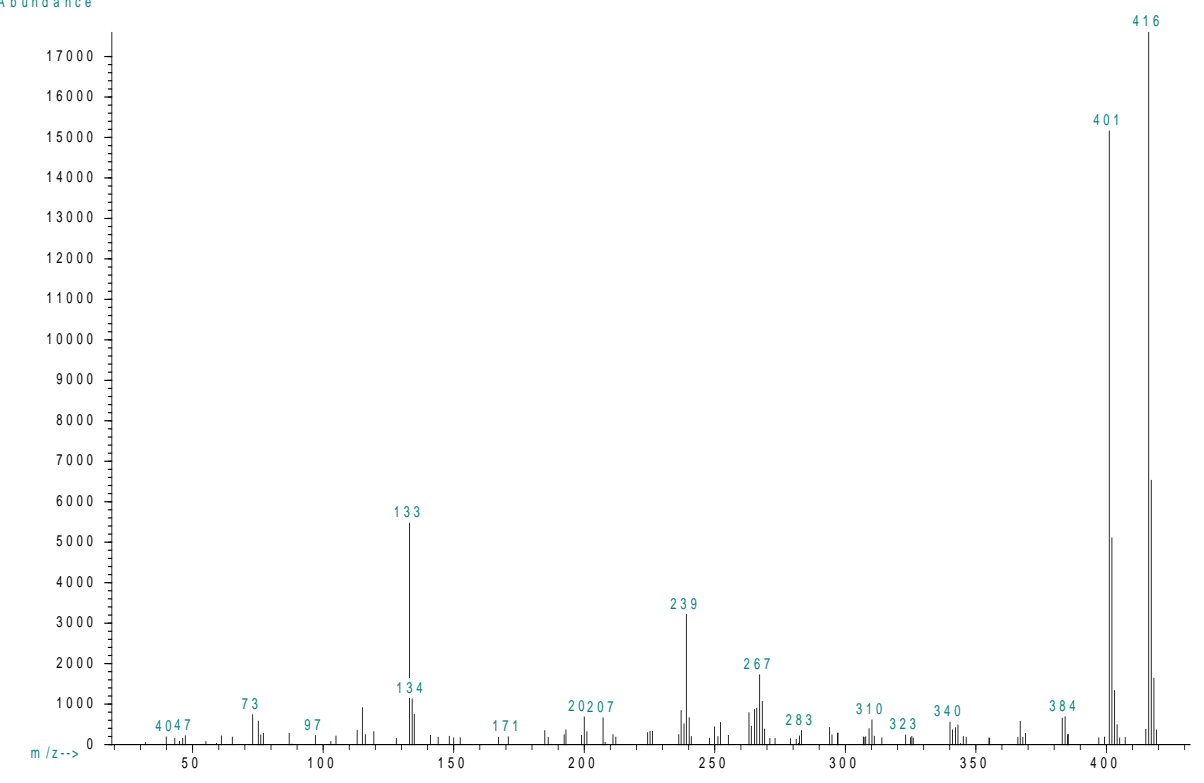

\section{Mass spectrum for RT 23.93 min:}

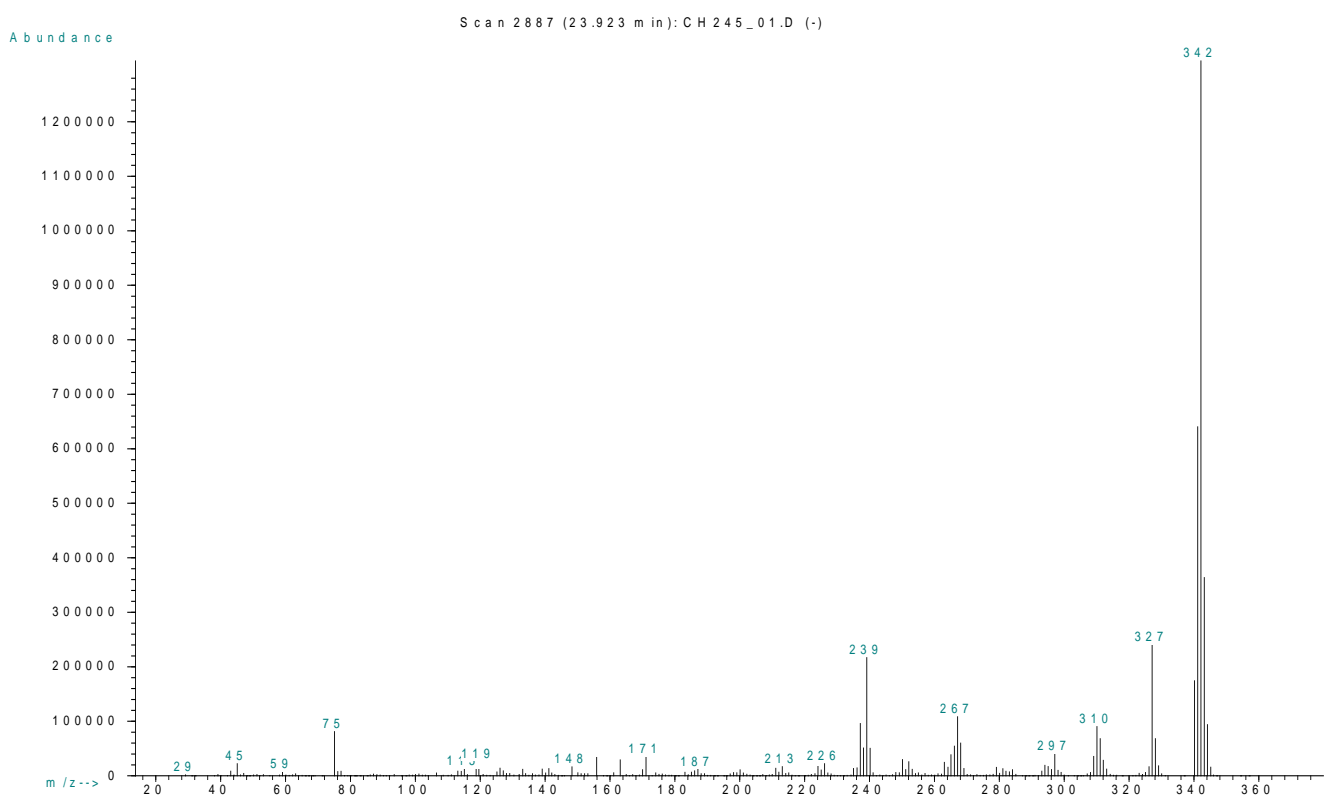

Mass spectrum for RT 25,48 min:

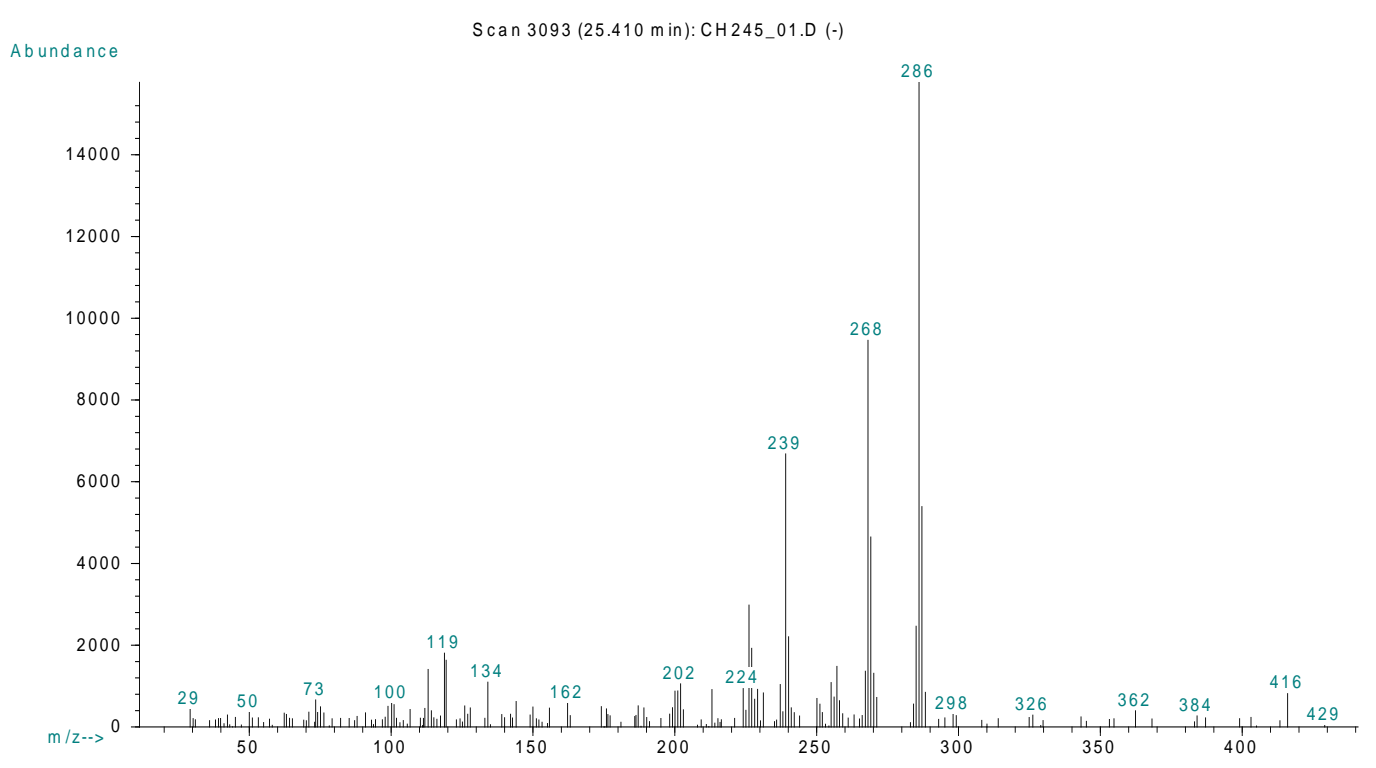




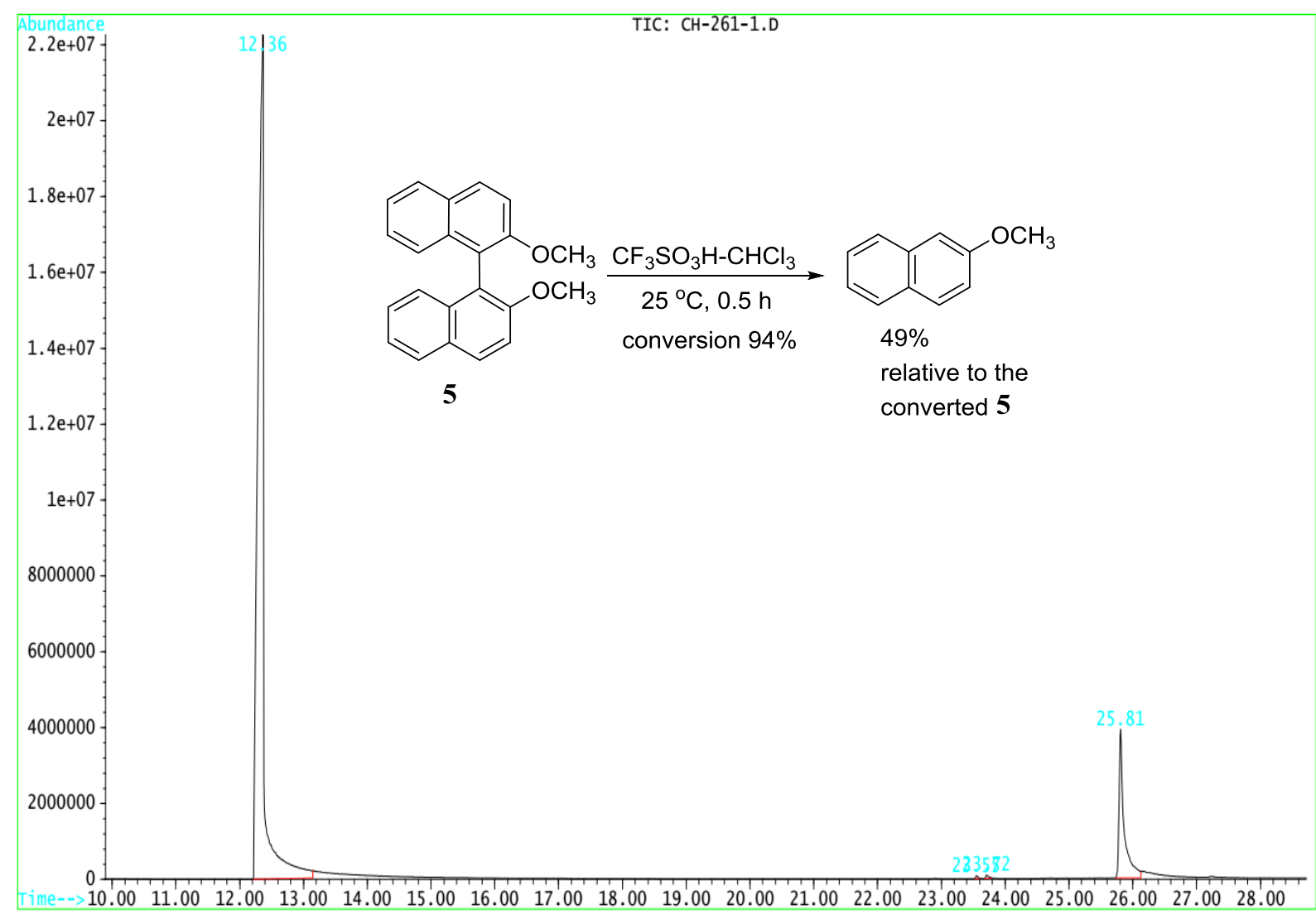

AcqMethod AN10_280

Misc Info: HP-5MS inj $280{ }^{\circ} \mathrm{C} 2 \mathrm{~m} 5010 \mathrm{deg} / \mathrm{min} 35 \mathrm{~min} 280{ }^{\circ} \mathrm{C}$

Retention Time Area Area \% Name

\begin{tabular}{ccccc}
\hline Total Ion Chromatogram & & & \\
12.364 & 154235635 & 87.580 & $\mathrm{MM}=158$ & Naphthalene, 2-methoxy- \\
23.552 & 220963 & 0.125 & $\mathrm{MM}=314$ & \\
23.716 & 287337 & 0.163 & $\mathrm{MM}=314$ & \\
25.807 & 21364581 & 12.131 & $\mathrm{MM}=314$ & $2,2^{\prime}$-Dimethoxy-1,1'-binaphthalene
\end{tabular}




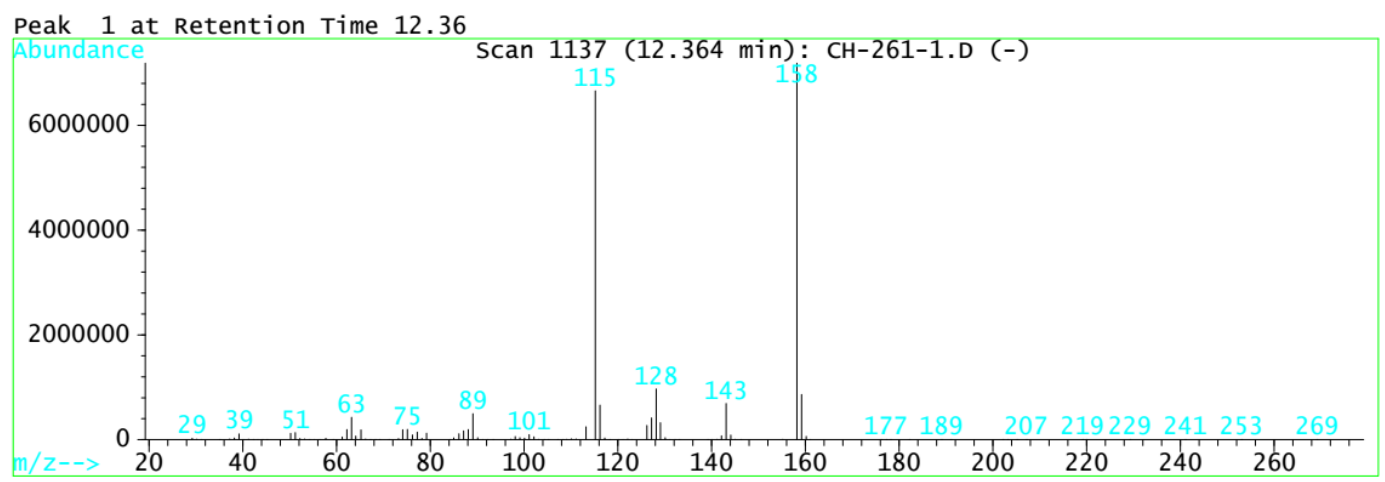

Peak 2 at Retention Time 23.5

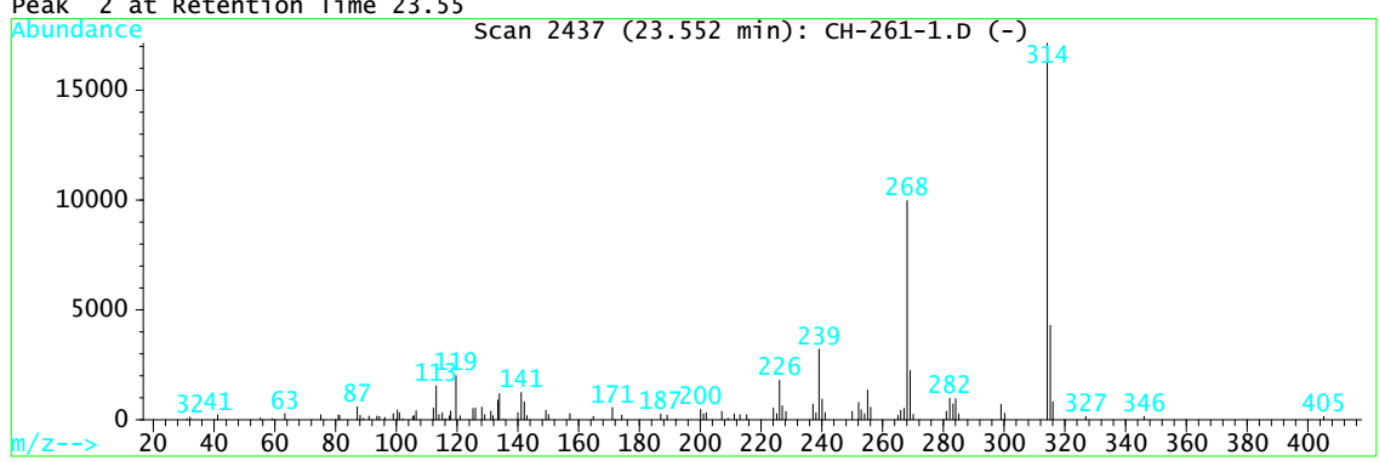

Peak 3 at Retention Time 23.72
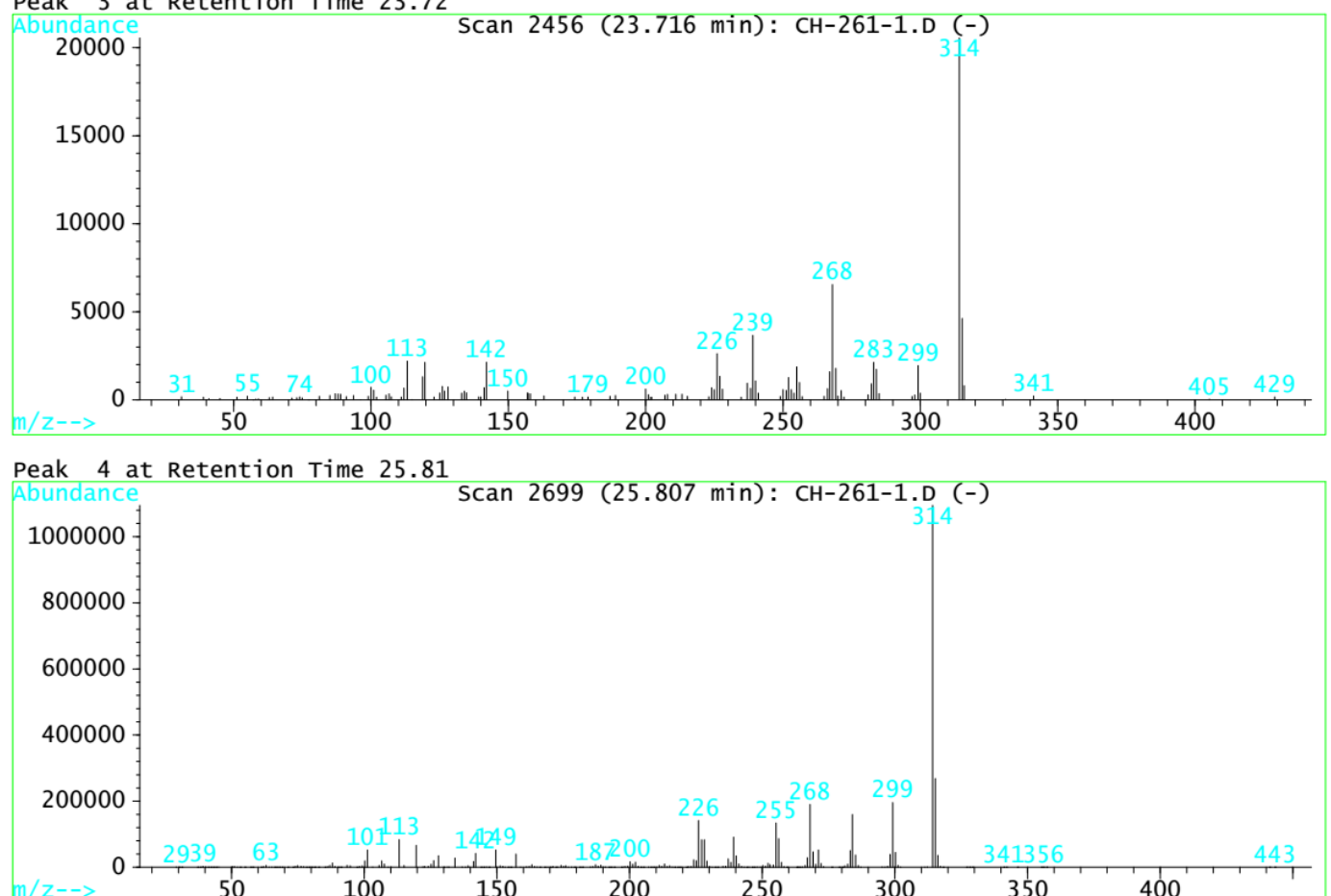